\title{
Progress in the mathematical modelling of visceral leishmaniasis
}

\author{
Kat S Rock ${ }^{\mathrm{a}, \mathrm{b}}$, Rupert J Quinnell ${ }^{\mathrm{c}}$, Graham F Medley ${ }^{\mathrm{d}}$, Orin Courtenay ${ }^{\mathrm{a}, \mathrm{b}, *}$ \\ ${ }^{a}$ School of Life Sciences, University of Warwick, Coventry, United Kingdom \\ ${ }^{b}$ Warwick Infectious Disease Epidemiology Research (WIDER), University of Warwick, \\ Coventry, United Kingdom \\ ${ }^{c}$ School of Biology, University of Leeds, Leeds, United Kingdom \\ ${ }^{d}$ Department of Global Health and Development, London School of Hygiene and Tropical \\ Medicine, United Kingdom
}

\begin{abstract}
The leishmaniases comprise a complex of diseases characterised by clinical outcomes that range from self-limiting to chronic, disfiguring and stigmatising, to life-threatening. Diagnostic methods, treatments, and vector and reservoir control options exist, but deciding the most effective interventions requires a quantitative understanding of the population level infection and disease dynamics. The effectiveness of any set of interventions has to be determined within the context of operational conditions, including economic and political commitment. Mathematical models are the best available tools for studying quantitative systems crossing disciplinary spheres (biology, medicine, economics) within environmental and societal constraints.

In 2005, the World Health Assembly and government health ministers of India, Nepal, and Bangladesh signed a Memorandum of Understanding to eliminate the life threatening form of leishmaniasis, visceral leishmaniasis (VL), on the Indian subcontinent by 2015 through a combination of early case detection, improved treatments, and vector control. The elimination target is $<1 / 10,000$ population at the district or subdistrict level compared to the current 20/10,000 in the regions of highest transmission.

Towards this goal, this chapter focuses on mathematical models of VL, and the biology driving those models, to enable realistic predictions of the
\end{abstract}

\footnotetext{
* Corresponding author

Email address: orin.courtenay@warwick.ac.uk (Orin Courtenay)
} 
best combination of interventions. Several key issues will be discussed which have affected previous modelling of VL and the direction future modelling may take. Current understanding of the natural history of disease, immunity (and loss of immunity), as well as stages of infection and their durations are considered particularly for humans, but also for dogs. Asymptomatic and clinical infection are discussed in the context of their relative roles in Leishmania transmission, as well as key components of the parasite-sandfly-vector interaction and intervention strategies including diagnosis, treatment and vector control. Gaps in current biological knowledge and potential avenues to improve model structures and mathematical predictions are identified. Underpinning the marriage between biology and mathematical modelling, the content of this chapter represents the first step towards developing the next generation of models for VL.

Keywords:

Visceral leishmaniasis, Mathematical modelling, Vector-borne transmission, Neglected tropical disease, Sandflies, Leishmania, Kala-azar, PKDL

\section{Contents}

1 Introduction $\quad \mathbf{3}$

1.0.1 Geographical distribution and disease burden . . . . . 3

1.0.2 Aetiological agents and transmission cycles . . . . . . 4

1.1 Disease in humans . . . . . . . . . . . . . . . 5

1.1.1 The clinical spectrum . . . . . . . . . . . 5

1.1.2 Natural history of VL disease . . . . . . . . . . . . . 5

1.1.3 Transmission: xenodiagnosis vs tissue and blood parasite loads . . . . . . . . . . . . . . . . . . 99 9

1.2 Disease in dogs . . . . . . . . . . . . . . . . . . . . . 11

1.3 Sandfly biology . . . . . . . . . . . . . . . . . . . . . . . . . . . . . . . . . . 14

1.3.1 Life expectancy . . . . . . . . . . . . . . . 14

1.3.2 Seasonality of sandflies . . . . . . . . . . . . . 15

1.3.3 Feeding behaviour .............. . . . . . 16

1.4 Control of human VL . . . . . . . . . . . . . . . . . . . . . . . . . . . . . 17

1.4.1 Treatment of VL . . . . . . . . . . . . . 17

1.4.2 Parasite loads in response to treatment . . . . . . . 18

1.4.3 Vector control . . . . . . . . . . . . . . 19

1.5 Control of ZVL . . . . . . . . . . . . . . . . . . . . . . 22 
2 Mathematical models of visceral leishmaniasis 23

2.1 Infection in humans . . . . . . . . . . . . . . . . . 24

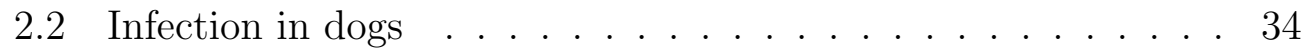

2.3 The role of sandflies . . . . . . . . . . . . . . 38

2.3.1 The basic reproduction number . . . . . . . . . . 42

2.3.2 Sandfly host choice . . . . . . . . . . . . . . . . . 44

2.3.3 Seasonality of sandflies . . . . . . . . . . . . . 45

3 Data-Model Interactions 50

3.1 Current data and initial models . . . . . . . . . . . 50

3.2 Future data and alternative sources . . . . . . . . . . . 52

4 Modelling Interventions 53

4.1 Humans . . . . . . . . . . . . . . . . . . . 53

4.1 .1 Diagnostics . . . . . . . . . . . . . . 53

4.1 .2 Treatment . . . . . . . . . . . . . . . 55

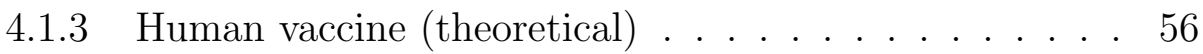

4.2 Vector . . . . . . . . . . . . . . . . . . 58

4.2 .1 On the ISC $\ldots \ldots \ldots \ldots \ldots$

4.2 .2 In Africa . . . . . . . . . . . . . . . . . . . . 59

4.2 .3 In Brazil . . . . . . . . . . . . . . . . . . . . . . . 59

4.3 Dogs . . . . . . . . . . . . . . . 61

4.3 .1 Diagnostics . . . . . . . . . . . . . . . 61

4.3 .2 Treatment . . . . . . . . . . . . . . . . . . . . . . 61

4.3 .3 Dog culling . . . . . . . . . . . . . . . . . . . 62

4.3.4 Dog vaccination . . . . . . . . . . . . . . . . . . . 64

4.4 Other animal hosts . . . . . . . . . . . . . . 65

5 Conclusions $\quad 65$

\section{Introduction}

\subsubsection{Geographical distribution and disease burden}

Endemic transmission of visceral leishmaniasis (VL) occurs in 79 countries on 5 continents where $>58,000$ new cases are officially reported each year; adjusted by the estimated levels of under-reporting (1.2- to 8.0-fold), the true annual case incidence is likely to be closer to 0.2-0.4 million (Alvar et al., 2012). Worldwide, 2.35 (95\% C.I. 2.18-4.90) million disability-adjusted life 
years (DALYs) are lost due to leishmaniasis, placing leishmaniasis second in mortality and fourth in morbidity burden among tropical diseases, where VL is ranked as the second largest parasitic killer after malaria (Mathers et al., 2007; Hotez et al., 2014).

Most (90\%) VL cases occur in 6 countries: India, Bangladesh, Sudan, South Sudan, Brazil and Ethiopia (Alvar et al., 2012), and 60\% of the global VL disease burden is on the Indian subcontinent (ISC) (WHO, 2002), where intense transmission is localised in Bihar state and Bengal (NE India), in the neighbouring Terai region of SE Nepal, and in the Mymensingh district in Central and West Bangladesh (Alvar et al., 2012). In Latin America, VL is less geographically focal, traditionally rural but with recent expansion into urbanised areas, notably in Brazil where approximately $90 \%$ of all VL cases in the Americas occur (Maia-Elkhoury et al., 2008).

\subsubsection{Aetiological agents and transmission cycles}

Leishmaniasis in humans is caused by more than 20 species of vectorborne protozoan flagellates of the genus Leishmania in two subgenera $L$. (Leishmania) and L. (Viannia). Visceral leishmaniasis, also known as "Kalaazar" (KA), is usually caused by members of the $L$. donovani complex ( $L$. donovani (syn. L. archibaldi) in S. Asia and E. Africa, and L. infantum (syn. L. chagasi) in C. and S. America, Mediterranean countries, C. Asia and parts of China) (Lukes et al., 2007). There are also rare reports of VL caused by infection with $L$. mexicana and $L$. tropica, which otherwise cause cutaneous leishmaniasis (Sacks et al., 1995; Barral et al., 1986).

Leishmania are transmitted by blood-feeding females of at least 30 Phlebotomine sandfly species in zoonotic transmission cycles involving one or more mammal reservoir host, with the exception of two species (L. donovani and L. tropica) that are transmitted in anthroponotic cycles. Domestic dogs are the sole proven animal reservoir of VL due to $L$. infantum; however proven transmission between sand fly vectors and wild hares (Lepus granatensis) in Madrid, indicate their potential as secondary local reservoirs (Molina et al., 2012). Despite a growing number of reports of $L$. donovani infections in dogs and other mammals in E. Africa and on the ISC (Bhattarai et al., 2010; Dereure et al., 2003; Alam et al., 2011; Sharma et al., 2009; Kenubih et al., 2014; Kassahun et al., 2015; Siriwardana et al., 2012) these hosts have yet to be shown to be epidemiologically relevant in maintaining transmission; differentiating incidental or "sink" host from reservoir or "source" hosts is required (reviewed by Quinnell and Courtenay (2009)). 


\subsection{Disease in humans}

\subsubsection{The clinical spectrum}

Leishmaniasis is characterised by a diversity of aetiological agents causing cutaneous (CL), mucosal (MCL) and visceral presentations dependent on the species or strain of Leishmania and on the integrity of the host's immune system. Infections may last for months to years, requiring specific and varied disease management strategies. VL is a systemic infection clinically characterised by prolonged fever, weight loss, splenohepatomegaly, pancytopenia and hypergammaglobulinaemia and anaemia. VL is almost always fatal if not treated, amounting to 20,000-40,000 deaths per year (Alvar et al., 2012), however the majority of VL-related deaths go unrecognised (Ahluwalia et al., 2003; Barnett et al., 2005; Collin et al., 2006). The risk of mortality from VL is exacerbated in the immunocompromised (e.g. HIV co-infections), patients with concomitant conditions (e.g. severe malnutrition) (Alvar et al., 2008; Cerf et al., 1987; Anstead et al., 2001; Dye and Williams, 1993; Bern et al., 2007), and those with limited treatment access due to conflict, famine or population displacement (Reithinger et al., 2007). Even amongst patients with access to VL treatment, the estimated case-fatality rate is 10\% (Alvar et al., 2012).

Post-kala-azar dermal leishmaniasis (PKDL) is the late and chronic development of skin lesions, usually following the apparent clinical cure of VL due to L. donovani in South Asia and Africa (Zijlstra et al., 2003). PKDL can develop months to years following apparent successful treatment for KA, or can develop without a previous KA episode. PKDL is not life threatening but is associated with disfigurement and social stigma (see Section 1.1.2).

\subsubsection{Natural history of $V L$ disease}

Transitioning from being susceptible to developing disease is a continuous process, but one of the challenges of mathematical modelling involves definition of discrete stages (compartments) and their interactions and overlaps. These stages are outlined below.

Susceptible. All naïve individuals across all age groups are potentially susceptible if exposed to Leishmania.

Individuals are not equally at risk of exposure and therefore infection, with significant heterogeneities dependent on environmental and socio-economic factors, especially related to poverty (Boelaert et al., 2009; Hasker et al., 2012; Alvar et al., 2012). Once infected the following clinical categories are recognised: 
Asymptomatic infection. The majority of infections are clinically asymptomatic (subclinical) in the long term. There is no standard definition of asymptomatic infection (Singh et al., 2014; Carneiro et al., 2011), though infection is indicated by detection of anti-Leishmania antibody by immunological tests (e.g. rK39 immunochromatographic test, direct agglutination test (DAT), enzyme-linked immunosorbent assay (ELISA)), by parasite detection by tissue culture, or by PCR. The variation in the sensitivity and specificity of these tests with respect to asymptomatic infection, hence their usefulness for surveillance, is difficult to determine (Cunningham et al., 2012; de Ruiter et al., 2014; Adams et al., 2013). Antibody presence for example can indicate current infection, past exposure, or relatively recent cure posttreatment for KA (see below). Thus, in a cross-sectional survey, individuals that mount an effective immune response will include individuals who do not progress to clinical KA.

With the exception of sporadic outbreaks (Ritmeijer et al., 2007), clinical development of leishmaniasis is rare relative to the number of infections. The ratio of infection to disease varies for $L$. infantum in Brazil (6.5:1, 9:1, 18.5:1), Spain (50:1), Iran (13:1); L. donovani in Bangladesh (4:1), India and Nepal (8.9:1), and L. donovani in Sudan (7:1,1.5:1), Kenya (6:1, 4:1), and Ethiopia (5.6:1, 11:1) (Evans et al., 1992; Badaro et al., 1986b,a; Ali and Ashford, 1994; Schaefer et al., 1995; Zijlstra et al., 1994; Hailu et al., 2009; Davies and Mazloumi Gavgani, 1999). Chapman et al. (2015) estimate that 20\% of asymptomatic infections develop into KA in Bangladesh, which is higher than other estimates, but utilises individual, longitudinal data over 3 years. The collective figures suggest that a larger proportion of $L$. donovani than $L$. infantum asymptomatic infections progress to disease but some of the differences between species and studies come from different definitions of asymptomatic infection and the use of different diagnostic tests; some studies use the Leishmania skin test (LST) as a marker of infection; othes use antibody detection tests of variable sensitivity and specificity. LST positivity is considered indicative of delayed-type hypersensitivity associated with a protective T-cell mediated response, whereas anti-Leishmania antibody is not considered protective. Immune markers characterising likely progression to disease are not fully defined but are influenced by host genetic factors, and the degree of T-cell mediated response (reviewed by Kedzierski and Evans (2014)). Well established risk factors influencing the probability of disease development include malnutrition, HIV co-infection, and poverty-related conditions (Bern et al., 2010; Boelaert et al., 2009; Alvar et al., 2008). Seroconversion or 
high Leishmania antibody titres indicate likely disease development, though are not specific markers (Hailu et al., 2009; Hasker et al., 2014; Sudarshan et al., 2014; Carneiro et al., 2011; Badaro et al., 1986a; Chapman et al., 2015). Additional challenges in defining asymptomatic status are discussed elsewhere (Singh et al., 2014; Carneiro et al., 2011).

Estimates of the pre-patent period, from detection of asymptomatic infection to development of KA varies between studies: in Sudan KA develops in 2-4 months (range: 2 weeks to 12 months) (Mueller et al., 2012); in Ethiopia this period is typically 3-8 months (Hailu et al., 2009); in Brazil, a mean 4.9 months (SD 3.6 months) (Badaro et al., 1986a), and on the ISC 3-6 months (Topno et al., 2010) and 142 days (Chapman et al., 2015).

Disease 1 - KA is defined on presentation of clinical signs, confirmed by a positive serological or/and parasitological assay. In ISC operational programmes, occurrence of at least 2 weeks fever and a positive rK39 test result triggers treatment. The interactions between exposure and disease can be inferred from the age distributions of KA cases. KA due to L. infantum (also known as "infantile VL") typically affects children <10 years (Badaro et al., 1986a; Davies and Mazloumi Gavgani, 1999), whereas KA due to $L$. donovani tends to be in older children and young adults $<30$ years in some locations, or older in others (Hailu et al., 2009; Mueller et al., 2012; Burza et al., 2014; Hasker et al., 2013). The age distribution will depend upon the exposure history of the population and will likely vary over the course of an epidemic cycle which is thought to be about 10-15 years on the ISC (Dye and Wolpert, 1988).

Disease 2 - PKDL occurs as a sequel to treatment and apparent cure of KA caused by $L$. donovani; it is clinically characterised as dermal macular, maculopapular, or nodular lesions on the face, upper arms and trunk (Zijlstra et al., 2003). People are usually otherwise well and the symptoms are less severe and distinct from KA with no associated mortality. Laboratory diagnosis of PKDL is challenging (Adams et al., 2013), and most cases are diagnosed on clinical grounds. PKDL is more common, less chronic and faster to develop the initial signs in parts of E. Africa than on the ISC: up to $56 \%$ of treated KA patients develop PKDL within 6 months (range: 013 months) in Sudan (Zijlstra et al., 2003; Musa et al., 2013), compared to $2-18 \%$ within $2-3$ years (range: 6 months to 32 years) on the ISC (Rahman et al., 2010; Singh et al., 2012; Uranw et al., 2011; Mondal et al., 2010a; Sultana et al., 2012). In Sudan, skin lesions tend to heal spontaneously in around $85 \%$ of patients and within 12 months, lasting a mean 9.7 months 
(SD 4.7 months; range: 2-28 months). In Bangladesh, around 50\% (48/98) of untreated PKDL patients showed spontaneous resolution within a median 19 months (Islam et al., 2013). Sudanese patients with lesions persisting $>12$ months show high anti-Leishmania antibody titres (DAT) and negative LST (Zijlstra et al., 2003; Musa et al., 2002). For 5-10\% of PKDL cases, there is no prior history of KA, and occasionally PKDL presents concomitant with KA (Zijlstra et al., 2003; Rahman et al., 2010; Islam et al., 2013). PKDL in Bangladeshi KA patients is more likely in younger ( $<15$ years) than older patients; with a median PKDL patient age of 12 years (Islam et al., 2013).

Infectious. For individuals to be confirmed as infectious/infective it is necessary to demonstrate their ability to infect the blood-feeding sandfly vector, which is known as xenodiagnosis. The infectious status of KA, PKDL and asymptomatic patients is unclear, representing a large gap in our current understanding of transmission pathways (see Section 1.1.3). A proportion of symptomatic L. infantum infections are infectious to sandflies, but their degree of infectiousness appears to be lower than for symptomatic $L$. donovani infections and PKDL (Quinnell and Courtenay, 2009). The higher infectiousness of HIV-co-infected individuals (Molina et al., 2003) suggests that this condition could become locally more relevant particularly where HIVco-infection case numbers are rising (Alvar et al., 2008, 2012). KA patients are usually treated soon after presentation at health facilities, so their period of potential infectiousness depends on care-seeking behaviour and diagnostic delays. However, relapses after treatment are not uncommon, which could lengthen the infectious period. The proportion of the population developing PKDL, though smaller, may delay seeking health care especially when symptoms are mild; also treatment may not be completed, for example if considered by patients too expensive or logistically difficult (Ozaki et al., 2011). In Bangladesh, current treatment guidelines call for a 120-day course of intramuscular injections of sodium stibogluconate (SSG) (Rahman et al., 2010). Thus a proportion of PKDL cases may remain in a chronic state and are potentially infectious for long periods.

Recovered and Immune. KA rarely occurs more than once in an individual, and treated cases appear to develop a strong immunity to disease (if not infection). However, it is not clear whether asymptomatic infection (that does not progress to disease) is protective against future infections and future disease. The LST is often used as a measure of the protective immunity and shows a variable duration. 98\% (42/43) of subjects were still LST positive 10 years post-cure from L. infantum in Maranhão, Brazil (Viana et al., 2011). 
In other regions of Brazil, only $4 \%$ and $16 \%$ were LST positive after 8-720 days and 4-9 years respectively post recovery from KA (Mayrink et al., 1971; Badaro et al., 1986a). Reasons for shorter positive skin test durations are likely to include absence of re-exposure to Leishmania to stimulate immunity, failing host immunity with age, and the technical quality and specificity of heterologous vs homologous LST antigens (Bern et al., 2006). Few to no new KA episodes have been reported amongst LST positives, and subsequent episodes are rare in others truly cured of KA (Burza et al., 2014; Hailu et al., 2009). Nonetheless, after clinical recovery, Leishmania antibodies persist for variable periods of up to 12 years (Gidwani et al., 2011; Bern et al., 2005), the variability partially determined by the degree of continued exposure and any persistent infection.

On the other hand, relapses in response to less optimal treatment regimes are not uncommon (see Section 1.4.1). To determine if immuno-competent past cases return to be partially or fully susceptible to infection and disease, it is essential to distinguish new infections from parasitological relapse, which is rarely studied. Relapse has been observed in 119/8,409 immuno-competent KA cases in a median 10.1 months (IQR: 7.1-13.8 months) with $32 \%$ of these relapsing $>12$ months post-successful treatment with 20mg/kg Ambisome, which is considered the current most effective treatment against KA on the ISC (Burza et al., 2014). Longitudinal follow-up studies are few and current parasite diagnostic tools may lack the required sensitivity to detect low-level residual infections.

\subsubsection{Transmission: xenodiagnosis vs tissue and blood parasite loads}

Since most VL infections are asymptomatic, quantifying their transmission potential relative to KA and PKDL cases is critical. Assuming that asymptomatically infected, KA and PKDL cases all contribute towards infection in sandflies, it is possible that transmission is primarily driven by the asymptomatic group, even if the relative infectivity of asymptomatics is much lower than KA or PKDL (see Section 2.1). PKDL patients have also been suggested to be an important reservoir based on observations that they consistently harbour L. donovani parasites in skin lesions, often do not seek or comply with long treatments, and can remain unsuccessfully treated for years (Nasreen et al., 2012; Verma et al., 2010; Thakur and Kumar, 1992). Cryptic reservoirs will become increasingly important to tackle as elimination is approached.

Quantifying infectiousness of host populations relies on xenodiagnosis or 
artificial exposure of sandfly vectors to host blood, but xenodiagnosis studies in particular are difficult on logistic grounds (e.g. maintaining sandfly colonies) and ethical grounds (e.g. exposing asymptomatic infecteds). Consequently xenodiagnosis studies are few, most are cross-sectional, and involve small numbers of hosts or vectors (reviewed by Quinnell and Courtenay (2009)). Historical xenodiagnosis studies demonstrate that both KA and PKDL patients can be infectious to sandflies: 18/21 L. donovani KA patients, and 4/4 PKDL (nodular lesions) infected $2-42 \%$ and $15-53 \%$ of exposed $P$. argentipes respectively, and $23 / 50 \mathrm{~L}$. infantum KA patients were infectious, on average, to 10-51\% of exposed Lu. longipalpis / P. perniciosus sandflies (reviewed by Quinnell and Courtenay (2009)). In contrast, 0/27 asymptomatic $L$. infantum infected individuals exposed to $L u$. longipalpis were infectious (Costa et al., 2000), but being LST positive, it is likely that they had resolved their infections and were more appropriately classified as recovered.

Molecular methods, particularly quantitative PCR (qPCR) to measure parasite burdens in skin and peripheral blood, i.e. accessible to blood-feeding sandflies, is more frequently being applied in population studies, and is often assumed to be a reliable marker of transmission potential. Unfortunately, the relationship between parasite load and xenodiagnosis outcomes are not well defined, and are likely to vary with the Leishmania-host interaction, clinical stage and kinetics of parasite tropism. It can be difficult to compare studies as the number of target gene copies (e.g. kDNA for preparing qPCR standards) varies between amastigotes and promastigotes, and even between strains (Weirather et al., 2011). Studies suggest that there exists a parasite density threshold above which canine and human infections are disproportionately infectious (Courtenay et al., 2014; Miller et al., 2014). In contrast, natural wildlife hosts (e.g. foxes and hares for $L$. infantum, rodents for $L$. braziliensis and L. tropica), in which infection is usually asymptomatic, show no apparent relationship between parasite loads and infectivity to sandflies (Courtenay et al., 2002a; Svobodova et al., 2003; Kassahun et al., 2015; Andrade et al., 2015; Molina et al., 2012).

Leishmania burdens in blood may, to some extent, differentiate asymptomatic and symptomatic infections and identify which infections are more likely to progress to disease, or to relapse post treatment (Sudarshan et al., 2014; Mary et al., 2006). L. infantum (Pourabbas et al., 2013; Mary et al., 2006; Moral et al., 2002; Biglino et al., 2010), and L. donovani (Miller et al., 2014; Sudarshan and Sundar, 2014) have been detected in blood in up to 
$58 \%$ of asymptomatic infections by a cross-sectional study. $14 \%$ of 4,695 asymptomatic Ethiopians were qPCR-positive in blood of which $3.2 \%$ had high genome equivalent counts (Miller et al., 2014), suggesting that distribution of parasitaemias is skewed and that perhaps the small proportion of asymptomatic infections with higher parasitaemia are more likely to develop disease and have a disproportional role in onward transmission. However of 44 Brazilian children with positive $\mathrm{qPCR}$ results at baseline, only 10 remained qPCR-positive 12 months later, showing a significant decrease in genome counts (dos Santos Marques et al., 2012). Only 4 of 25 asymptomatic PCR-positive individuals remained PCR-positive 12 months later in SE Brazil (de Gouvea Viana et al., 2008). In sharp contrast, 83\% (80/97) of asymptomatic individuals living in an active transmission region of Brazil tested PCR-positive during 6 years follow-up (tested three times by PCR) (Carneiro et al., 2011).

In PKDL patients, parasite loads are reported to be greater in nodular compared to macular or papular lesions (Nasreen et al., 2012; Verma et al., 2010, 2013), whereas samples from asymptomatic skin from PKDL patients in Bangladesh $(n=20)$ tested negative compared to the $95 \%$ positivity of active lesions (Nasreen et al., 2012). PCR positive results were obtained in $35 \%(8 / 23)$ of slit skin samples taken from PKDL lesions in the same region (Islam et al., 2013). Any contribution of PKDL to onward transmission may differ substantially between Africa and the ISC due to differences in PKDL onset, progression and clinical aspects as described above (Section 1.1.2). Lesion type (i.e. parasite loads), treatment (see Section 1.4.1) and healthcare-seeking behaviours are also likely to influence infectious duration. Assuming non-biased selection of study subjects, the majority $[77 \%$ $(106 / 138)$ and $97 \%(107 / 110)]$ of PKDL patients' cutaneous lesions involved macules and papules, and only 3\% (3/110 patients) and 4\% (5/138 patients) were nodular forms; the remainder were mixed or complex lesion types (Nasreen et al., 2012; Islam et al., 2013). Also higher loads were recorded in more chronic PKDL cases ( $>3$ years history) compared to acute $(<3$ years history) PKDL cases suggesting an increased parasite load with disease duration (Verma et al., 2010).

\subsection{Disease in dogs}

L. infantum infection in the domestic dog also causes a spectrum of clinical disease, from asymptomatic infection to severe, fatal disease (reviewed by Solano-Gallego et al. (2009)). The burden of disease due to zoonotic visceral 
leishmaniasis (ZVL) in dogs is difficult to quantify, since prevalence varies at both large and small geographical scales, and diagnostic tests have varying sensitivity and specificity. In Western Europe a review of canine serological surveys found the overall seroprevalence in 500,000 sampled dogs to be $23 \%$, with a median value of $10 \%$ (Franco et al., 2011). The prevalence of disease will be lower, but the true prevalence of infection will be much higher, since serology is known to have low sensitivity for detecting asymptomatic infection (Baneth et al., 2008). The use of molecular methods has shown that the real prevalence of infection can be up to $80 \%$, in areas where $<30 \%$ were seropositive (Solano-Gallego et al., 2001; Lachaud et al., 2002). Similarly, longitudinal studies using multiple diagnostic methods have shown that the incidence of infection can be very high, with a mean time to infection of 115 days in one Brazilian study (Quinnell et al., 1997), and incidence of 40-92\% over the course of a transmission season in Europe (Dye et al., 1993; Oliva et al., 2006). Indeed in some regions the incidence appears to be steadily rising (Antoniou et al., 2009).

The course of infection is best examined using longitudinal studies of natural infection. The few examples include a series of studies of kenneled beagles exposed to natural infection in Italy, and a cohort study of owned mixed-breed dogs in Amazon Brazil (Quinnell et al., 1997, 2001; Courtenay et al., 2002b; Oliva et al., 2006; Foglia Manzillo et al., 2013). Such studies have confirmed that infected dogs can be qualitatively divided into two groups: resistant and susceptible. In the Italian studies, around $50 \%$ of infected dogs either showed no evidence of established infection but had low levels of anti-leishmanial antibodies, or were PCR-positive with low or no anti-leishmanial antibody response, and did not progress to clinical disease. The other $50 \%$ of dogs, after a variable period of asymptomatic infection, showed progressive infection, with increasing parasite burdens and serological titres and development of clinical signs (Gradoni, 2015). Clinical signs in canine VL are varied and non-specific: the most common signs are weight loss, lymphadenopathy and a range of cutaneous signs (Baneth et al., 2008; Foglia Manzillo et al., 2013). The proportion of dogs that develop clinical disease is harder to estimate in Brazil, since dogs in many endemic areas are generally malnourished and suffer from a range of other infections presenting similar clinical signs. Resistance and susceptibility is associated with differences in cellular immune responses, but little is known about the factors that underlie this variation in outcome between dogs (Baneth et al., 2008; Solano-Gallego et al., 2009). Age, sex, nutrition and co-infection may play 
a role, but variation in outcome is seen in even well-nourished experimental dogs. Host genetics are known to be important, even within pedigree breeds (Quilez et al., 2012).

Few studies have examined infectiousness, but it is likely that all susceptible dogs become infectious, to a greater or lesser degree. In the Brazilian study, about half of infected dogs became infectious, an average of 3 months after seroconversion, i.e. about 6 months after infection (Courtenay et al., $2002 b$ ). This and other xenodiagnostic studies have shown that the proportion of infectious dogs varies with clinical status and also geographical area. Meta-analysis of 7 published studies showed that the proportion of polysymptomatic dogs that are infectious is very high (0.80), but that a proportion of asymptomatic dogs are also infectious (0.29) (Quinnell and Courtenay, 2009). The proportion of infectious dogs was higher in European than Latin American studies (0.86 vs 0.45 ), which could indicate a greater susceptibility of an Old World sandfly vector, Phlebotomus perniciosus, to infection compared to the major New World vector, Lutzomyia longipalpis. However, it remains unclear what the role of resistant dogs is in disease transmission. Although some asymptomatic dogs are infectious, it is not possible to tell from cross-sectional studies whether these dogs are susceptible dogs in the early stages of infection, or resistant dogs. The Brazil cohort study suggested that most asymptomatic infectious dogs did go on to develop disease, and that truly resistant dogs have only a small role in transmission (Courtenay et al., 2002b).

Indeed there is a high degree of heterogeneity between symptomatic dogs in their level of infectiousness, with very different contributions to transmission. Of 42 infected dogs (18 infectious) in the Brazilian cohort study, 7 (17\%) highly infectious dogs were responsible for $>80 \%$ of the transmission events (Courtenay et al., 2002b), and other studies show that $15-44 \%$ of infected dogs are responsible for $>80 \%$ of transmission (Travi et al., 2001; Molina et al., 1994). These results have been corroborated by studies of tissue parasite burdens across dogs, which show similarly high levels of heterogeneity (Courtenay et al., 2014).

Diagnosis of infection in dogs has been reviewed in (Solano-Gallego et al., 2009). PCR, particularly qPCR, is the most sensitive diagnostic technique, and is usually the first test to be positive early after infection (Oliva et al., 2006; Quinnell et al., 2001). Detecting infection in resistant asymptomatic dogs is difficult, as a proportion of PCR-positive resistant dogs do not go on to seroconvert, and antibody levels are low and vary through time. Susceptible 
dogs appear to all seroconvert, though the time taken for seroconversion can vary considerably. Clinical signs are not sufficient for diagnosis due to their low specificity. Distinguishing infectious from non-infectious dogs is also difficult, though parasite burden assessed by qPCR may provide a useful measure of the level of infectiousness (Courtenay et al., 2014).

In addition to dogs and humans, a number of both domestic and wild mammals can be naturally infected with L. infantum (reviewed by Quinnell and Courtenay (2009)). In general, there is as yet no strong evidence that hosts other than the domestic dog are responsible for significant human infection, but further studies, particularly xenodiagnostic studies, are needed. Other domestic or sylvatic reservoirs have thus been ignored in modelling to date. However, there is recent evidence that hares, rather than dogs, have been responsible for a localised outbreak of several hundred human cases in Madrid (Molina et al., 2012).

\subsection{Sandfly biology}

Sandflies are small biting flies of the family Psychodidae. Only adult females take blood meals, while both adult males and females feed on plant sugars; larvae are terrestrial (see Killick-Kendrick (1999) for a review of sandfly biology). The vector of VL on the ISC is Phlebotomus argentipes, though $P$. papatasi is suspected to be a secondary vector. In Sudan the main vector is $P$. orientalis, with other vector species occurring in Kenya and China (Killick-Kendrick, 1999). The most widespread vector of ZVL in the New World is Lutzomyia longipalpis, which is likely to be a complex of several species, while other species ( $L u$. evansi and $L u$. cruzi) may be important locally (Lainson and Rangel, 2005; Quinnell and Courtenay, 2009). In the Old World, there are at least a dozen sandfly vectors for L. infantum, with

$P$. perniciosus and $P$. ariasi the major vectors in the western Mediterranean (Killick-Kendrick, 1999).

\subsubsection{Life expectancy}

Sandflies have a relatively long generation time, taking at least a month to develop to adulthood (Killick-Kendrick, 1999). A key epidemiological parameter is the adult life expectancy, since to transmit infection female sandflies need to survive long enough to take at least two blood meals and to allow parasites to develop to infectivity (the extrinsic incubation period). The length of the gonotrophic cycle varies between species, being around 3 days in Lu. longipalpis, around a week for P. ariasi, 5 days for P. argentipes 
and 5-11 days for P. papatasi (Srinivasan and Panicker, 1992; Lainson et al., 1977; Palit et al., 1990).

There have been few studies of the life expectancy of adult sandflies of any species under natural conditions. Adult life expectancy can be approximated from field measures of physiological age using the parous rate. The proportion of parous flies (flies that have laid eggs) approximates the proportion of flies that are older than 1 gonotrophic cycle. This method has been used to estimate the life expectancy of $P$. ariasi in France as around 6 days, that of Lu. longipalpis in Brazil as 2.4 days, and that of P. papatasi in India as 14 days (Dye et al., 1987, 1991; Srinivasan and Panicker, 1992). The parous rate of Lu. evansi in Colombia was comparable to that of Lu. longipalpis in Brazil (Travi et al., 1996), while that of Lu. longipalpis in Colombia was higher (Ferro et al., 1995), suggesting an average life expectancy of up to a week. Mark-release-recapture methods can also be used to directly estimate life expectancy of sandflies, such as the L. braziliensis vector Lu. (Nyssomyia) neivai (Casanova et al., 2009). However, for VL vectors their use has been limited to studies of dispersal, which have shown incidentally that adult $L u$. longipalpis can survive for up to 7-8 days (Dye et al., 1991; Morrison et al., 1993a)), and P. ariasi for up to 28 days (Killick-Kendrick and Rioux, 2002).

\subsubsection{Seasonality of sandflies}

There is a pronounced seasonal variation in numbers of VL vectors, both in the Old and New World. In tropical regions, sandflies are usually present in every month, but with up to a 10 -fold variation in sandfly numbers across the year. On the ISC, numbers are lowest in December and January, when temperatures are lowest, and typically highest in summer and the post-Monsoon period; numbers correlate positively with temperature, but correlations with rainfall are more variable (Ghosh et al., 1999; Picado et al., 2010a). In Amazon Brazil, sandfly numbers peak at the end of the dry season, and this variation in population size is reflected in a marked variation in the incidence of canine infection across the year (Quinnell et al., 1997; Kelly et al., 1997). The seasonal pattern in other areas of Brazil is different, with highest numbers in the more arid NE Brazil typically occurring during or after the rainy season (Sherlock, 1996; Deane and Deane, 1962), though some areas show no clear seasonal pattern (Costa et al., 2013b). In the Mediterranean area seasonality is more marked, with no adults present during the winter months and sandflies surviving this period as larvae in diapause. In Italy the period of sandfly activity runs from May to October/November, with highest 
sandfly numbers from June to August (Rossi et al., 2008). In mountainous NW Iran, the VL transmission season lasts for the 4-5 month summer (), as also observed in the Mediterranean (Gavgani et al., 2002). Seasonal patterns are also often reflected in the parous rate, infection rate and other important entomological parameters.

\subsubsection{Feeding behaviour}

The sandflies that transmit VL have fairly broad host choice. Lu. longipalpis will readily feed on a range of vertebrate hosts, and host choice is determined largely by host size and accessibility (Quinnell et al., 1992; Morrison et al., 1993b). Lu. longipalpis is not considered to be strongly anthropophilic, though this may vary across its range, as members of the $L u$. longipalpis species complex differ in their degree of attraction to human odours in the laboratory (Rebollar-Tellez et al., 2006). In the Mediterranean, both $P$. perniciosus and $P$. ariasi are similarly opportunistic feeders on mammalian and avian hosts (Guy et al., 1984; de Colmenares et al., 1995). P. argentipes and $P$. orientalis also feed on a range of mammals, both humans and domestic animals, particularly cattle, buffalo and goats (Garlapati et al., 2012; Gebresilassie et al., 2015; Palit et al., 2006)), though P. papatasi appears to be predominantly anthropophilic (Palit et al., 2006). This lack of host specificity means that domestic animals probably play an important role in the epidemiology either as reservoirs and/or as sources of blood. On the ISC, large numbers of sandflies are found peridomestically in cattle sheds, while in Brazil, large numbers are often found in animal shelters such as chicken sheds. Since neither cattle nor chickens are hosts of Leishmania, this raises the possibility that domestic animals may reduce the infection rate in humans by diverting sandflies from humans or dogs and lowering both the feeding and infection rate (zooprophylaxis) (Alexander et al., 2002; Bern et al., 2010). These effects could be magnified since both Lu. longipalpis and $P$. argentipes are thought to have a lek-based mating system, where males aggregate on hosts and attract females using pheromones (Dye et al., 1991; Lane et al., 1990). Conversely, the presence of domestic animals could increase the biting rate by acting as a maintenance host for the vector and thus increasing vector numbers (Alexander et al., 2002; Bern et al., 2010) (zoopotentiation); both positive and negative associations between cattle and risk of infection have been reported on the ISC (Bern et al., 2010). 


\subsection{Control of human $V L$}

\subsubsection{Treatment of $V L$}

The pentavalent antimonials (sodium stibogluconate [SSG] and meglumine antimoniate), the mainstay treatment for VL since the 1940s, are generally effective, with the notable exception of parts of South Asia where up to $60 \%$ treatment failure in Bihar, India (Mondal et al., 2010b; Sundar, 2001; Sundar et al., 2000) and neighbouring Nepal (Rijal et al., 2007, 2003) have been reported and associated with parasite resistance (Lira et al., 1999; Downing et al., 2011). Antimony treatment requires 30 day hospitalisation for daily injections imposing a catastrophic economic burden on affected families (Ozaki et al., 2011). Antimony is highly toxic, particularly in malnourished and very young or old patients, hence diagnostic test specificity is of particular importance. In the past two decades of drug development (reviewed by den Boer et al. (2009)), conventional forms of amphotericin $\mathrm{B}$, liposomal preparations of amphotericin B, miltefosine (MIL), and paromomycin have been registered in various countries to treat VL. Conventional amphotericin B has replaced antimonials as the first-line treatment for VL in parts of India (Olliaro et al., 2005), following trials showing that it is highly effective and safe (Sundar et al., 2003, 2004).

Paromomycin (PM) (formerly known as aminosidine), is an aminoglycoside antibiotic with good anti-leishmanial activity, showing high levels of efficacy and safety, and low relative cost, and was registered in India in 2006. MIL is the only oral drug against VL registered for use in India since 2002 and has been adopted by the Indian Ministry of Health in parts of Bihar (Matlashewski et al., 2011) and more recently in Nepal. However it requires 28 days of oral treatment, and as it is teratogenic it cannot be used to treat pregnant or lactating women, which hinders its use in roll-out programmes. Reported treatment failure rates in immunocompetent patients are up to $21 \%$ (and 33\% amongst children) within 12 months in Nepal and 5-10\% within 6 months in India, the former attributed to a low-dose-response drug exposure rather than in vitro parasite resistance (Dorlo et al., 2014; Sundar et al., 2012; Rijal et al., 2013). The 2005 Memorandum of Understanding for a "VL Elimination Programme" on the ISC was partially based on replacement of antimony by MIL as the first-line treatment (Mondal et al., 2009). Single-dose liposomal amphotericin B (AmBisome®) showing significantly diminished renal toxicity, is now considered the best available therapy against $L$. donovani on the ISC (WHO, 2010a), at a negotiated affordable 
price (Matlashewski et al., 2011; de Melo and Fortaleza, 2013), and has recently been adopted in some regions of the ISC (Burza et al., 2014).

In E. Africa, monotherapies have proven less efficacious and with greater regional variability than seen in S. Asia (den Boer et al., 2009; Mueller et al., 2007; Khalil et al., 2014). Since AmBisomeß requires a cold chain and is very expensive with limited availability, it is considered a second line treatment, but under continued evaluation (Khalil et al., 2014). One concern with monotherapies is the potential development of parasite resistance, particularly MIL and PM, because they require long treatment courses, lowering compliance. MIL resistance is reported to result from a single point mutation in the MIL transporter at the Leishmania plasma membrane, associated with reduced accumulation of cell reactive oxygen which causes apoptotic death (Seifert et al., 2007; Das et al., 2013). Parasite resistance against amphotericin B is thought to be less likely (Lachaud et al., 2009). The safety, tolerance and effectiveness of combination therapies (reviewed by van Griensven et al. (2010)) now trialled in India and E. Africa (see also Musa et al. (2012); Sundar et al. (2008); Chunge et al. (1990); Thakur et al. (2000); Melaku et al. (2007); Seaman et al. (1993)) are encouraging, and expected to help slow the rate of parasite resistance to treatment, to reduce treatment duration, to enhance compliance, and to be cost-effective (Musa et al., 2012; Hamad et al., 2010; Croft et al., 2006). Following further efficacy trials (Khalil et al., 2014; Musa et al., 2012), WHO recommended 17-day treatment with PM combined with SSG as a first line treatment against VL in E. Africa (Khalil et al., 2014). In Brazil, amphotericin B and liposomal amphotericin $\mathrm{B}$ are currently recommended by the Ministry of Health with multicentre clinical trials underway (de Melo and Fortaleza, 2013). However, no current or proposed treatment would be suitable for mass drug administration, which emphasises the importance of parallel development of accurate and operational diagnostics.

\subsubsection{Parasite loads in response to treatment}

In relation to transmission, the choice of an appropriate VL treatment regime to ensure parasite clearance and to eliminate potential infectivity to sandflies is essential. Leishmania burdens in treated VL patients generally decline during treatment (reviewed by Kip et al., 2014; see also Pourabbas et al., 2013; Mourão et al., 2014; Mary et al., 2004, 2006; Aoun et al., 2009; Sudarshan et al., 2011). However inadequate drugs, doses and durations may result in parasite persistence (e.g. $<50 \%$ and $<60 \%$ tissue parasite clearance 
respectively immediately after, and 6 months after, completion of low dose PM treatment in Sudan (Hailu et al., 2010)). Relapses with a resulting rise in parasite loads are not uncommon (e.g. Pourabbas et al. (2013); Mourão et al. (2014)), and even in KA patients considered clinically cured without recorded relapse, residual parasites may persist e.g. in 11/21 Iranian patients treated with antimony (Glucantime) Pourabbas et al., 2013; 2/6 patients treated with SAG (Verma et al., 2010), and 4/48 Brazil children under various treatment regimes (Mourão et al., 2014).

\subsubsection{Vector control}

\section{Indoor Residual Spraying (IRS)}

Vector control by IRS using DDT or pyrethroid insecticides is a key component of national VL control campaigns, with mixed success in reducing infection incidence via purposeful IRS against Leishmania vectors, or as a benefit of the spraying campaigns against malaria vectors (Alexander and Maroli, 2003; Ostyn et al., 2008). IRS is effective against adult vectors that blood-feed indoors (endophagic) or rest indoors between blood-feeding and searching for an oviposition site (endophilic). IRS is not expected to impact on exophilic and exophagic vectors, nor immature stages; though sandfly breeding sites are largely unknown (Alexander and Maroli, 2003). Measurable entomological indicators of control success include reductions in human landing rates, blood-feeding success, or human blood index, and reduced vector survival (lower parous rates), as demonstrated for a wide range of New World (Davies et al., 2000; Feliciangeli et al., 2003a,b; Courtenay et al., 2007; Falcao et al., 1991), and Old World sandfly species (Benzerroung et al., 1992; Reyburn et al., 2000; Joshi and Rai, 1994; Kaul et al., 1994; Mukhopadhyay et al., 1996; Joshi et al., 2009). Insecticide deterrency (sometimes called repellency $c f$. excito-repellency) induces vectors to avoid contact with insecticide-treated surfaces, thus potentially diverting infectious bites onto unprotected hosts; however this is not a usual feature of current insecticide products for vector control.

The only research-based cluster randomised trial to evaluate IRS was conducted in India, Bangladesh and Nepal, though interventions varied in insecticides used (DDT in India, deltamethrin in Bangladesh, and alphacypermethrin in Nepal) and in the wall surfaces treated (Joshi et al., 2009). House and cattle shed spraying reduced the indoor $P$. argentipes overall density by $72 \%$, an effect consistent across sites, though with mixed results reported in other studies in India (Kumar et al., 2009; Picado et al., 2010b; 
Dinesh et al., 2008a). Since 1991-2, the Indian Kala-azar Control Program has promoted two IRS interventions per year using DDT; in Nepal IRS operational programmes use predominantly lambda-cyhalothrin (Joshi et al., 2003), whereas in Bangladesh IRS was very sporadic to non-existent up to 2010 (Bern and Chowdhury, 2006; Mondal et al., 2008). In Brazil, reactive IRS using lambda-cyhalothrin, cypermethrin or deltamethrin is conducted on detection of a KA case (da Saúde Secretaria de Vigilância em Saúde Departamento de Vigilância Epidemiológica, 2006). IRS government-run campaigns against VL vectors appear to be absent in E. Africa.

Despite some encouraging entomological research outcomes, IRS as deployed under the umbrella of operational VL control programmes has had little to no impact on VL incidence, either in India (Picado et al., 2012; Ostyn et al., 2008), Nepal (Joshi et al., 2006), Bangladesh (2006) or Brazil (de Souza et al., 2008). However robust IRS evaluation studies are limited (Quinnell et al., 2001; Picado et al., 2012), though guidelines to monitor IRS in the context of the VL elimination programme are now in place (WHO, 2010b). In India, resistance of $P$. argentipes to DDT and pyrethroids is patchy (Ostyn et al., 2008; Dinesh et al., 2008b). The Indian and Nepalese routine IRS activities are considered suboptimal due to financial and logistical constraints, poor quality spraying practices and low community compliance (Chowdhury et al., 2011; Picado et al., 2012; Coleman et al., 2015). In Brazil, spraying lambda-cyhalothrin in animals sheds where the vector $L u$. longipalpis congregates was shown to increase the numbers of Lu. longipalpis in household dining huts (attached to houses), indicating the importance of complete coverage (Kelly et al., 1997).

\section{Insecticide-impregnated nets and materials}

Insecticide-treated nets (ITNs) treated with synthetic pyrethroids have been shown to be effective against mosquito vectors and malaria incidence (Lengeler, 2004). ITNs against many species of sandflies similarly demonstrate knockdown and toxicity (reviewed by Alexander and Maroli (2003); Ostyn et al. (2008)), and have been shown to be efficacious in reducing the incidence of both anthroponotic CL and zoonotic CL (Jalouk et al., 2007; Alten et al., 2003; Reyburn et al., 2000; Moosa-Kazemi et al., 2007; Davies et al., 2000; Kroeger et al., 2002; Yaghoobi-Ershadi et al., 2006; Tayeh et al., 1997).

In eastern Sudan, lambda-cyhalothrin impregnated bednets against the vector $P$. orientalis reduced the incidence of $\mathrm{KA}$ to $1.6 \%$ in a single intervention village compared to $12.4 \%$ in the control village, but lacked replication (Elnaiem, 1996). Provision of deltamethrin impregnated bednets to 
114 follow-up Sudanese villages in response to the 1999-2001 VL epidemic, reduced VL by 59\%; the greatest impact was reported to be 17-20 months after the distribution of the nets (Ritmeijer et al., 2007). An intervention trial against $P$. argentipes in Bangladesh indicated that widespread bednet impregnation with slow-release insecticide may reduce the frequency of VL, however the study was inconclusive because it was not randomised and lacked sufficient replicates (Mondal et al., 2013).

Whilst few ITN trials have been conducted against VL vectors, a notable exception is the cluster randomised controlled trial known as the "Kalanet Trial" (www.kalanet.info) which distributed deltamethrin impregnated longlasting bednets to 26 paired intervention and control villages in highly endemic foci in India (16 pairs) and Nepal (10 pairs) (Picado et al., 2010b). The control arm continued existing practices (irregular IRS, use of untreated nets, and treatment of VL), which were accounted for in the final analyses. After 24 months follow-up, there was no significant reduction observed in seroconversion in intervention $(347 / 6372=5.4 \%)$ compared to control $(345 / 6319=5.5 \%)$ residents, or in the risk of developing KA $(37 / 9829=0.38 \%)$ verses controls $(40 / 9981=0.40 \%)$. However the intervention did significantly reduce the risk of malaria $(\mathrm{RR}=0.46$ [95\% C.I. 0.28-0.77]). The authors suggest that possible reasons for the failure to impact on VL transmission are that $P$. argentipes may be more exophilic (lives outdoors) and exophagic (feeds outdoors), and zoophilic (prefers feeding on animals), than previously considered as the effect of the intervention on vector density was measured only indoors (Picado et al., 2015). Thus the entomological impact of bednets on sandflies depends largely on both host and vector behaviours (Courtenay et al., 2007); further issues about feasibility are discussed elsewhere (Courtenay et al., 2007; Ostyn et al., 2008; Picado et al., 2015).

High coverage with ITNs may result in reduced vector infection rates (i.e. a mass effect) in anthroponotic cycles though this has not been empirically demonstrated. The role of ITNs in combating zoonotic transmission is limited: for anthroponotic VL, ITNs are expected to provide personal protection against infective bites, and theoretically to impact on the transmission cycle. For zoonotic organisms (where humans are not reservoirs) ITNs will protect and impact on indoor vectors, but are not expected to directly impact on the transmission cycle. 


\subsection{Control of $Z V L$}

In areas where human cases are comparatively rare, such as the Mediterranean region, ZVL is considered as a veterinary rather than a human public health problem, and there are typically no national measures to control infection. In other areas, such as Brazil, national ZVL programmes are in place. In Brazil, the national control programme is focused on (i) detection and culling of seropositive dogs, (ii) indoor residual spraying (IRS) of houses and animal shelters and (iii) diagnosis and treatment of human cases. These methods have been successfully used in China to control infection, although the relative contributions of vector and dog control are not known (Zhi-Biao, 1989; Costa, 2011). In Brazil such measures have shown little or no efficacy, as the number of human and canine cases have not declined. As a result, the rationale for these methods, especially dog culling, has been increasingly questioned on theoretical, practical and ethical grounds (Otranto and Dantas-Torres, 2013; Dye, 1996; Costa, 2011; Courtenay et al., 2002b). A number of logistical problems have been identified with the dog culling programme: coverage is often low, the serological diagnostic tests used are relatively insensitive, and there are long delays between sampling and culling (reviewed by Quinnell and Courtenay (2009)). Culled dogs are also rapidly replaced with naïve, uninfected dogs (Nunes et al., 2008). These concerns have been partly addressed by the recent introduction of a new rapid diagnostic test, which should reduce delays and may improve sensitivity. Unfortunately, the efficacy of either IRS or dog culling remains unknown, due to a lack of well-designed, large field trials (Romero and Boelaert, 2010; Quinnell and Courtenay, 2009).

Other potential control methods include protection of dogs with topical insecticides, protection of humans with bednets (see Section 1.4.3), pheromone traps for sandflies, dog treatment, or canine vaccines. Synthetic pyrethroids applied to dogs as topical formulations or in impregnated collars have been shown to be highly effective in protecting dogs against sandflies, with effects lasting for up to 8 months for collars but only 3-4 weeks for topical "pour-on" applications. Under field conditions, randomised controlled trials have shown such methods to also be highly effective in protecting individual dogs against infection, with efficacies of 50-100\% (reviewed by Quinnell and Courtenay (2009); Otranto and Dantas-Torres (2013)). However, only one randomised, community-level control trial against human infection has been carried out, showing deltamethrin-impregnated collars fitted to dogs in NW Iran to reduce canine infection by $53 \%$ reduction in canine infection 
and by $38 \%$ in humans (Gavgani et al., 2002). Lu. longipalpis males produce a pheromone that attracts females to lekking sites on hosts, and field trials have shown that lures using synthetic pheromone are highly attractive to both male and female sandflies, and could potentially be used in control programmes (Bray et al., 2014).

Treatment of dogs with anti-parasitic drugs is widely used in a veterinary context in Europe, but it is not used in control programmes. Indeed, treatment of dogs with drugs used to combat human infection is banned in Brazil, in order to reduce the possibility of drug resistance. The clinical response of dogs to treatment varies according to the initial condition of the dog, but clinical cure is often seen in dogs with mild to moderate infection (SolanoGallego et al., 2009). However, treatment does not result in parasitological cure, and dogs are likely to remain infectious, though at a reduced level. In contrast to drugs, canine vaccines may provide a very useful tool for disease control, and much effort has been invested in the development of a canine ZVL vaccine. Three vaccines are now commercially available: Leishmune and Leish-Tec in Brazil, and CaniLeish in Europe. Relatively little has been published on the efficacy of these vaccines, particularly from phase III field trials, and a number of published studies have methodological issues (Wylie et al., 2014). There is evidence from several studies that Leishmune is effective against clinical disease, and maybe also against infection, with reported efficacies of 76-100\% (Oliveira da Silva et al., 2001; Borja-Cabrera et al., 2002; Nogueira et al., 2005; Felix de Lima et al., 2010). A single field study of CaniLeish shows no significant effect on infection, but a $68 \%$ reduction in the proportion of dogs with clinical disease (Oliva et al., 2014). There have been no published phase III studies yet for Leish-Tec. An anti-disease (as opposed to anti-infection) vaccine would be suitable, provided that the vaccine reduced parasite loads to a level below that at which parasite transmission occurs. It has also been suggested that Leishmune may have specific transmission-blocking effects (Saraiva et al., 2006). Vaccines may also have immunotherapeutic effects when given to already infected animals (BorjaCabrera et al., 2004).

\section{Mathematical models of visceral leishmaniasis}

The number of mathematical models of VL in either humans or animals is low, in particular for VL on the Indian subcontinent (see Table 5) where the majority of elimination efforts are currently being targeted. The exist- 
ing models are quite varied both in the biological details that they capture, but also in their underlying mathematical structure, however it is noted that they are mostly still ODE models which neglect spatial effects. As might be expected with models of an NTD, many lack realistic parameterisation that arises through model fitting to high-quality data. Several of the models do address pertinent questions related to the control of disease through intervention such as detection and treatment and vector control, but none directly address the WHO goal of elimination of disease as a public health problem by 2020 nor generate the likely time to full elimination given the current prevalence of disease and existing/proposed control strategies. These existing models of VL pave the way for more sophisticated models of this disease that utilise and extend prior frameworks.

The two forms of visceral leishmaniasis, caused by L. donovani and L. infantum, are separated both geographically and by the way in which they are modelled. L. donovani on the Indian subcontinent is considered to be anthroponotic with only humans and sandflies playing a role in the transmission cycle. Consequently models of KA on the ISC do not feature non-human populations explicitly, although it is recognised that the presence and relative density of alternative hosts may ultimately affect the human biting rate. ZVL (caused by L. infantum) primarily affects Brazil in addition to the Mediterranean region and parts of the central Asia and China. In these regions an animal population (dogs) not only forms part of the transmission cycle, but it is also thought to drive it. In regions of East Africa affected by $L$. donovani and on the ISC, other animals have been shown to be infected though their contribution towards transmission remains untested.

\subsection{Infection in humans}

In order to understand the transmission dynamics of VL it is important to be able to identify the natural progression of the disease in humans. From this biological starting point, a mathematical model can be developed.

The first model of VL in humans by Dye and Wolpert (1988) was a simple compartmental model in which individuals in the human population were considered to be either susceptible to VL infection, infected (and infectious) or have experienced the disease and were recovered and immune from reinfection. This basic structure has been well studied for many diseases and is known as an SIR progression model (see Figure 1) (Keeling and Rohani, 2008). Despite its relative simplicity, such a model can be analysed easily and provides clues towards understanding observed phenomena. For example, Dye and Wolpert 
were able to show that if VL is considered to have an immunising effect, this alone could generate the pronounced inter-epidemic periods seen historically (1880-1940) in India. Other factors such as treatment, disasters (including earthquakes) and other diseases such as influenza governed the size, but not timing of these epidemics.

Figure 1: Simple model of SIR VL progression in humans as used by Dye and Wolpert (1988) with progression between stages at rates $\lambda_{H}$ and $\phi_{H}$. Here the red box denotes the only infectious stage.

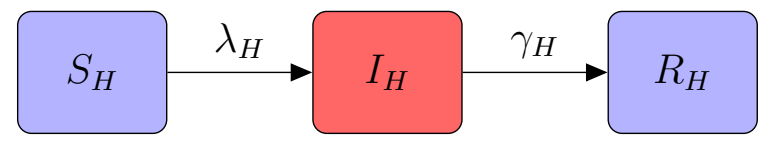

Although biological understanding of VL has advanced since 1988, it was 22 years after this original model that an improved dynamic transmission model was developed to examine contemporary VL on the ISC. The model by Mubayi et al. (2010) sought to quantify the underreporting bias of KA cases in Bihar, India by combining a transmission model with data (from 2003 and 2005) from the most affected 21 of 38 districts in Bihar. In addition to the SIR compartments, the model includes stages for: latent infection where individuals have been infected with Leishmania but are not yet infectious to sandflies; and treatment compartments where diseased patients attend either public or private centres before recovery is possible (see Figure 2). This is a type of SEIR (susceptible - exposed - infected - recovered) model. The proportion of those attending public facilities is key to answering the amount of under-reporting as only public health centres were required to report KA cases. Within the model, any privately treated patients are assumed to be unreported. In 2006-2009 a retrospective study indicated that the proportion of cases receiving treatment for VL in government health facilities was low (less than $30 \%$ for adults, and as low as $13 \%$ for 5 to 10 year olds (Gidwani et al., 2011)) indicating that there could be a large amount of under-reporting.

To include more realistic latent, infectious and treatment periods, Mubayi et al. (2010) make use of the "linear-chain trick", a mathematical technique where each of these compartments is partitioned into subcompartments. Using this method generates Erlang-distributed (gamma-distributed with the shape parameter, $k \in \mathbb{N}$ ) periods rather than exponential distributed periods (MacDonald, 1978). The number of subcompartments is dictated by the shape of the probability density function for that epidemiological period; for 
example, Mubayi et al used $n=16$ compartments for the latent stage but only $m=2$ for the infectious stage because the variance was much higher for duration of infectious period compared to the variance of latent period. Although this methodology provides a useful mathematical tool for modelling the variability in the latent and infectious periods, the subcompartments do not correspond directly to biological processes.

Figure 2: Model of VL progression considered by Mubayi et al. (2010) including Erlang distributed latent, infectious and treatment stages with red denoting the infective classes and yellow, the treatment in either public or private health centres.

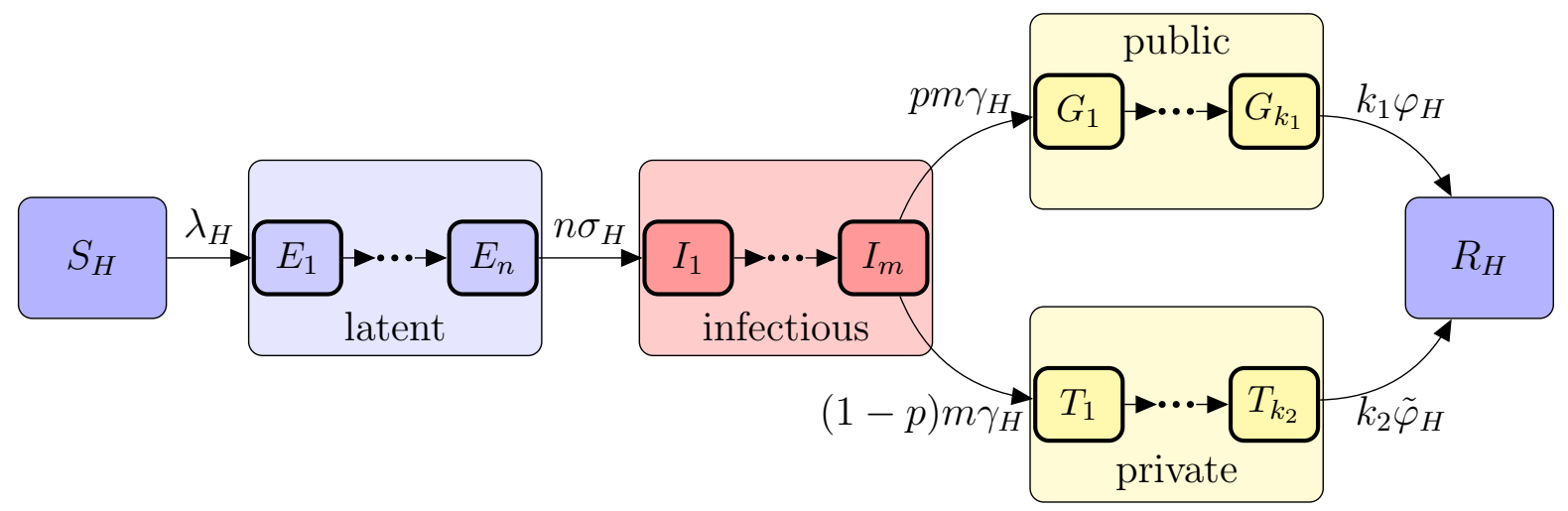

Under this model the percentage of underreporting is $100(1-p)$ where $p$ is the proportion of patients who attend public health centres. The mean number of reported cases per month is the influx into the first disease compartment of the model assuming that all new cases are immediately reported from public health centres but never from private ones:

$$
\text { reported cases }=p m \gamma_{H} I_{m}
$$

This equation may be rearranged to find $p$ based on the endemic equilibrium prevalence (denoted by stars):

$$
p=\frac{\text { reported cases }}{m \gamma_{H} I_{m}^{*}}
$$

where $I_{m}^{*}$ can be expressed as a function of model parameters.

Using this methodology it was found that there was on average $88 \%$ underreporting of infection in 2003 which decreased to $71 \%$ in 2005 . Underreporting reached as high as $96 \%$ (in Jahanabad district) and a positive correlation was found between underreporting and population density (Mubayi 
et al., 2010). This modelling demonstrates the huge extent of underreporting that has likely occurred in the past due to differences in reporting between health facilities and shows that caution must be taken with standard reported incidence.

These first two dynamic models of VL progression in humans were tailored to address specific questions; the first regarding inter-epidemic periods and the second addressing underreporting. However there are other aspects of $L$. donovani infection which are absent from these models, most notably the inclusion of asymptomatic infections and PKDL.

The first transmission model to include this disease state was by ELmojtaba et al. (2010a) who modelled infection in Sudan; this model was later extended (ELmojtaba et al., 2010b, 2012). The compartmental model was a typical SIR-type model with an extra infected (and infectious) compartment for PKDL patients (see Figure 3). In this framework, infected patients who are treated either fully recover (with probability $(1-f)$ ) or develop PKDL (with probability $f$ ) following KA. The authors assumed that PKDL was linked to effectiveness of treatment and so $(1-f)$ is proportional to effectiveness. PKDL patients are assumed to be treated or to spontaneously recover (with rate $\eta_{H}$ ) and once again become non-infectious.

Figure 3: Model of VL progression adopted by ELmojtaba et al. (2010a,b, 2012) with both KA $\left(I_{H}\right)$ and PKDL $\left(P_{H}\right)$ classes and red denoting the infective classes. Following KA, patients either develop PKDL with probability $f$ or recover immediately.

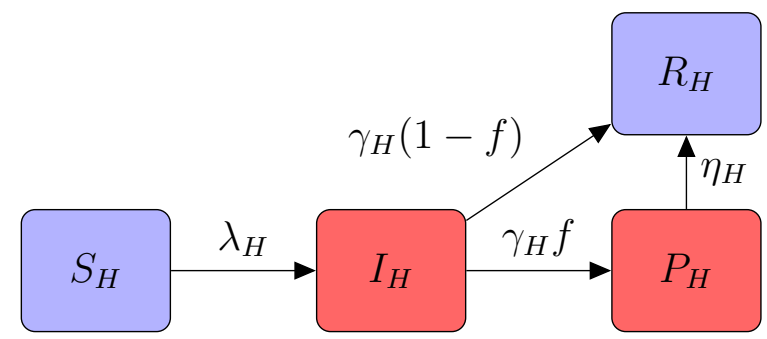

By creating this extra compartment, the authors were able to analyse the effect of treatment for both KA and PKDL, including both rate and effectiveness (this is discussed further in Section 4). As this model was developed for the Sudan, it may be reasonable to model instantaneous transition from KA infection to PKDL due to the relatively short time (0-6 months) between the two, however, this can be amended to incorporate a dormancy period between the two symptomatic stages (see Figure 4) and would make the model 
structure more appropriate for the ISC where the onset of PKDL can be 3 years after KA treatment.

Figure 4: Model of VL progression with both KA $\left(I_{H}\right)$ and PKDL $\left(P_{H}\right)$ classes with intermediate dormancy $\left(D_{H}\right)$. Red denotes the infective classes. The probability of progression to PKDL from KA is the same as before (Figure 3), however the time from treatment to PKDL onset is on average $\xi^{-1}$.

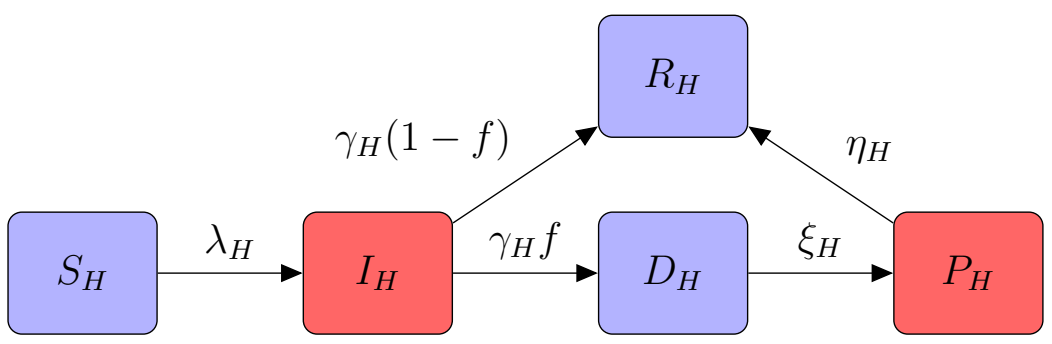

The dynamic model (and variants thereof) by Stauch et al. (2011, 2012, 2014) includes a dormancy period between KA and PKDL, however they also add many more compartments to the human progression including classes for first and second-line treatment and asymptomatic infection. Conceptually the Stauch progression is not dissimilar to the approach illustrated in Figure 4, with an additional asymptomatic class occurring before KA symptoms, possible spontaneous recovery from asymptomatic infection, and waning immunity from the recovered class to susceptible. These additions are shown in Figure 5.

Figure 5: Model of VL progression with asymptomatic VL $\left(A_{H}\right)$, KA $\left(I_{H}\right)$ and PKDL $\left(P_{H}\right)$ classes with intermediate dormancy $\left(D_{H}\right)$. Red denotes the infective classes.

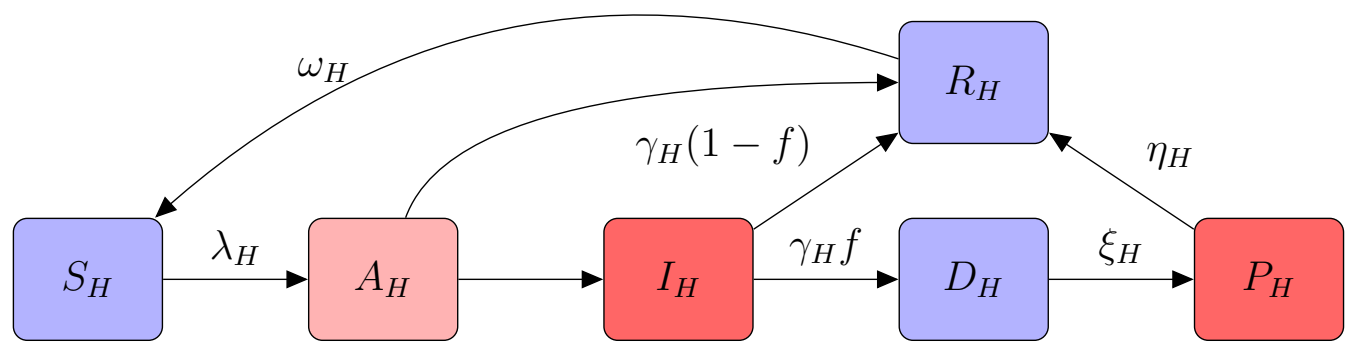

Using this model of disease progression, Stauch et al then create further subcompartments which are tied to diagnostic test results, symptoms and 
Figure 6: Compartmental diagram showing VL progression paths in humans (adapted from (Stauch et al., 2011)). Underneath their corresponding results for 3 different tests for VL is shown (as given by Stauch et al. $(2011,2012,2014)$ ). Blue boxes denote individuals who do not play a role in transmission of disease to the vector. The red boxes denote those that do or might (with asymptomatics contributing much less (40- to 80-fold less) than KA or PKDL patients.

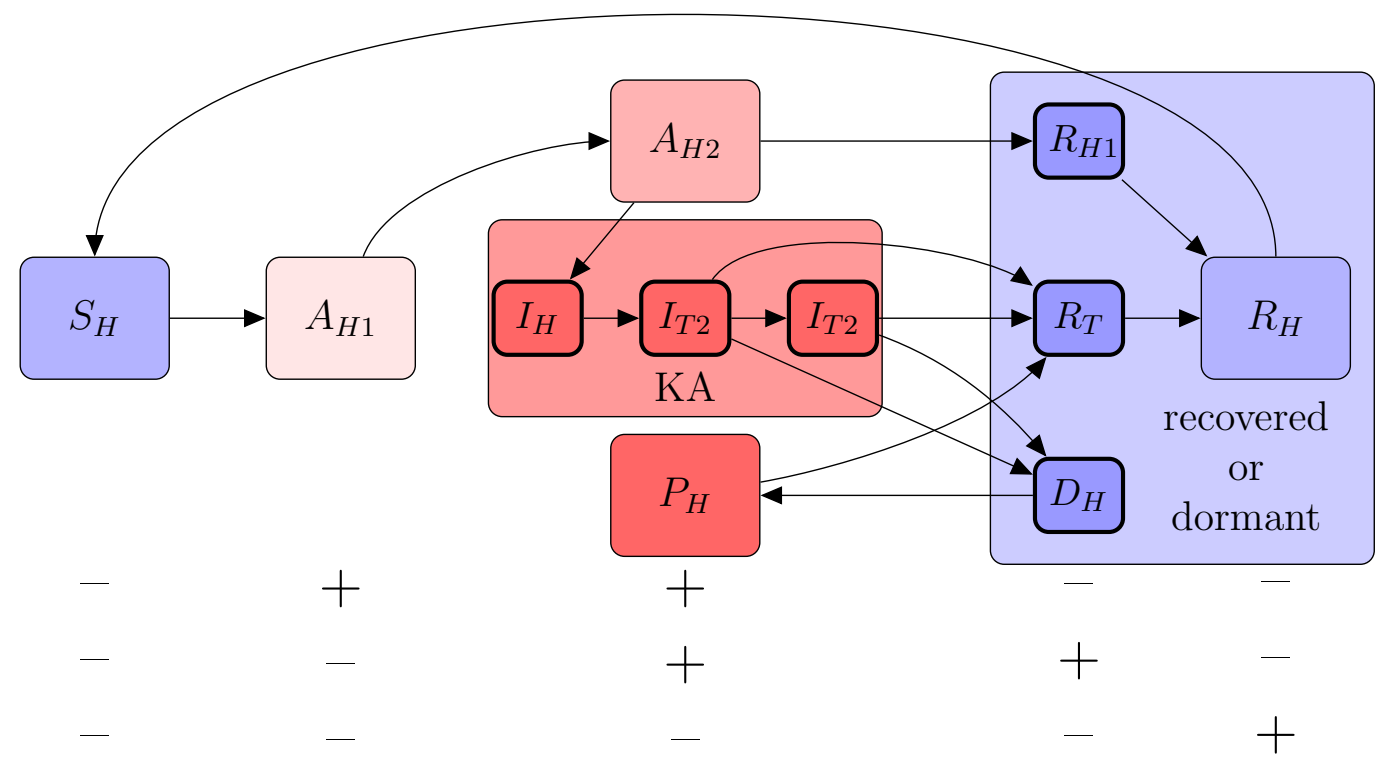

treatment. For example, asymptomatically infected people are now stratified into two separate classes, denoted here by $A_{H 1}$ and $A_{H_{2}}$. The first of these groups of people have positive PCR but negative DAT results, whereas the second group test positive for both. KA patients also have PCR/DAT positive tests, however the presence of symptoms is used to distinguish them from asymptomatics, and the treatment they are receiving (no treatment, first-line and second-line) assigns their subcompartment. Figure 6 shows how the new compartments are amended from the assumed progression (Figure 5) and the results of diagnostic tests.

This model was developed from and fitted to cross-sectional data from the Kalanet study in India and Nepal (see Section 1.4.3). The model does not take account of the sensitivity or specificity of the three diagnostic tests used in the trial. Unlike other models of VL, this model allows for waning immunity, which is the loss of immunity to Leishmania infection over a period of 
time. Through fitting to the data, the average time spent immune (in the $R_{H}$ compartment) was just 307 days, which is shorter than might be expected. The model also included the impact of HIV co-infection upon the dynamics of VL, with a greater proportion of infected people developing symptoms if they were HIV-positive compared to HIV-negative. As co-infection of these two pathogens was not part of the Kalanet study, the HIV prevalence had to be taken from regional estimates.

Through tying human VL progression to results of diagnostic results, Stauch et al have raised an important question: how should asymptomatically infected individuals (who are PCR positive) be modelled within the transmission cycle? Such people are infected with Leishmania parasites but their role in infection of sandflies is uncertain (see Section 1.1.3). In the model of Stauch et al "late stage" asymptomatic were assumed to have a reduced infectivity by some factor, called here $\varepsilon$, compared to symptomatically infected, KA individuals (who have infectivity $p_{V}$ towards sandflies). "Early stage" asymptomatic were assumed to have even lower infectivity of $0.5 \varepsilon$, although the justification for this is not clear. Through model fitting the reduced infectivity parameter, $\varepsilon$, was estimated to be 1/40 and so this would indicate that asymptomatics are 40 to 80 -fold less infectious than KA or PKDL patients.

The model results suggest that even at this low infectivity, asymptomatic individuals are a large enough group to sustain transmission and, therefore, that treating KA and PKDL patients, alone is unlikely to be sufficient to reduce transmission enough to achieve elimination as a public health problem. This is the only dynamic model to examine asymptomatically infected humans. It points towards a need to fit models that include asymptomatic individuals to other data sets, but also emphasises the need for xenodiagnostics. Ultimately, even if the individual infectivity of asymptomatics is low, this should not be dismissed, as if there are many such individuals, it may hamper intervention efforts.

Chapman et al. (2015) took an alternate approach to previous modellers to infer both rates of disease progression and durations of disease states by developing a multistage Markov model of the natural history of VL infection. Utilising data from a longitudinal study in Fulbaria upazila of Bangladesh, they concluded that disease durations were longer than those estimated by Stauch et al including a waning immunity of 1110 days (988-1247 95\% CI). Chapman et al. (2015) also found that progression from asymptomatic infection to KA was high (14.7\%) compared to Stauch et al (0.33\%). 
Given these long duration estimates and high rates of progression to KA, it would be expected that a dynamic transmission model similar to that of Stauch et al may produce different results when parameterised with the updated values. In particular, asymptomatics may not be responsible for maintenance of transmission as found by Stauch et al. (2011), however as asymptomatic infection is found to progress more often to KA, understanding the dynamics of this subclinical, infected class of individuals will remain important.

Figure 7: Uncertainties in the human infectious reservoir. Here all individuals who may contribute towards infection in the vector are shown in the large red box. "Infectious", $I_{D}$ individuals are always assumed to be infective to sandflies and "dormant", $D_{H}$ (before relapsing to PKDL) are thought to never be infective but do still have low parasitaemia.

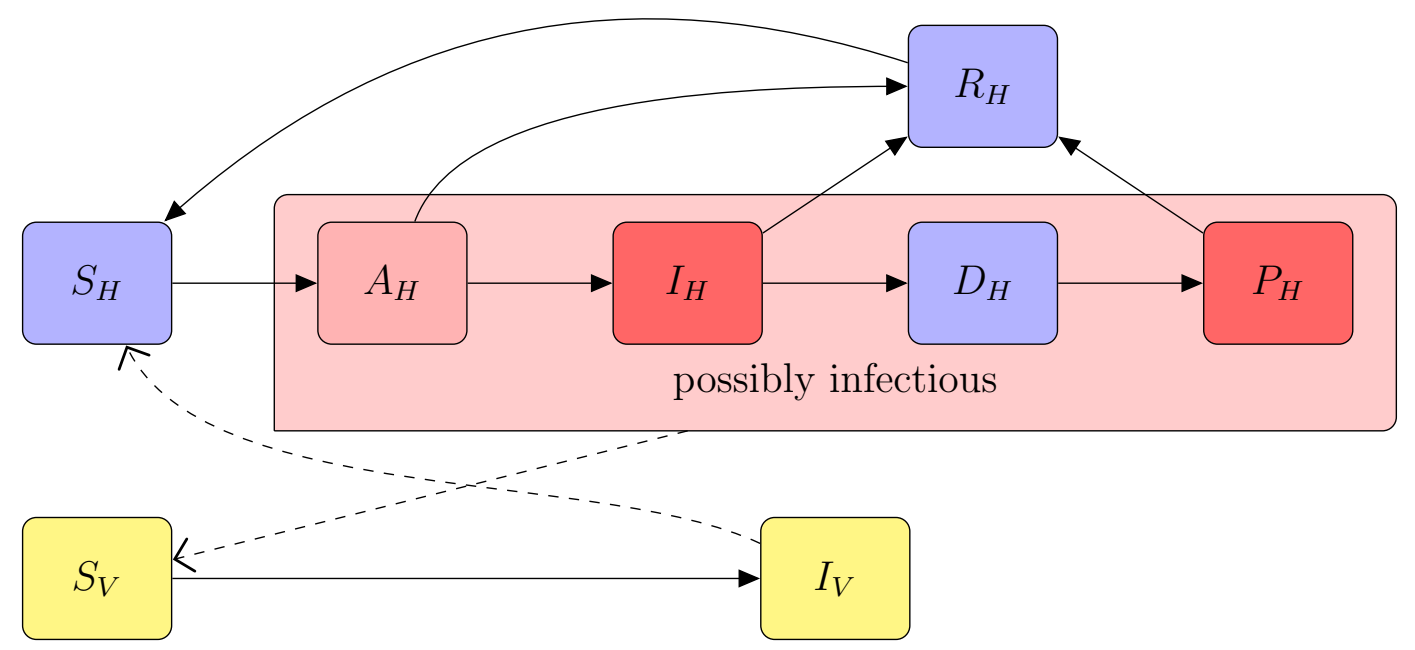

Different models have made very different assumptions both about disease progression and parameterisation. The huge variability in parameters and their values may be attributed both to the underlying model structures and also the uncertainty in many factors in transmission and progression, such as rates or probabilities, which are either hard to measure or vary greatly by region. Consequently this will impact on the cross applicability of results.

The mathematical modelling of L. infantum infection in humans is limited to SIR-type models, where people are only considered to be "spill-over" hosts from a main transmission cycle between sandflies and dogs. As PKDL does not occur for this species of parasite, this human disease progression is a reasonable assumption. In many endemic settings where L. infantum is 
found, the prevalence in dogs is much greater than in humans, and so little transmission is assumed to occur from humans. 
Table 1: Parameters associated with human VL progression and their notation used here and across modelling papers. All rates are per capita unless stated otherwise.

\begin{tabular}{|c|c|c|c|c|c|}
\hline Parameter & Notation & 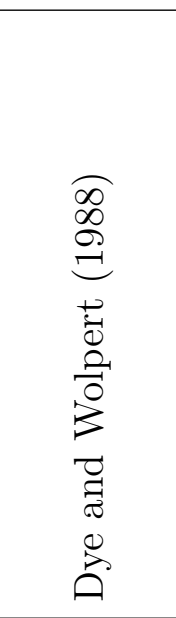 & 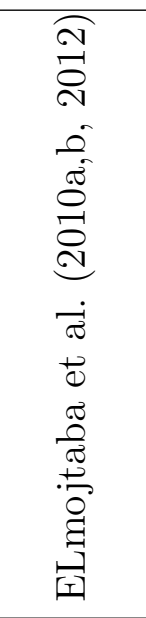 & 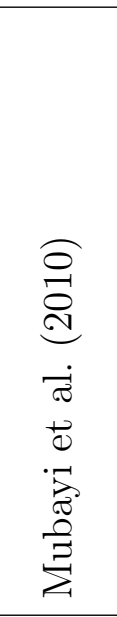 & 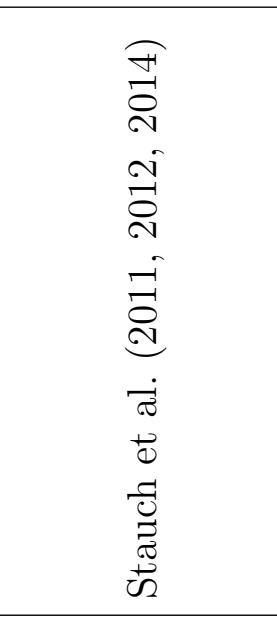 \\
\hline $\begin{array}{c}\text { total birth/recruitment rate } \\
\text { death rate } \\
\text { rate of infection (ROI) } \\
\text { rate of becoming symptomatic } \\
\text { probability of PKDL after KA } \\
\text { rate of developing PKDL after recovery } \\
\text { disease-induced mortality } \\
\text { waning immunity }\end{array}$ & $\begin{array}{c}\mu_{H} \\
\lambda_{H} \\
\sigma_{H} \\
f \\
\xi_{H} \\
\delta_{H} \\
\omega_{H}\end{array}$ & $\begin{array}{c}(a+e) N \\
b \\
c I / N^{\ddagger} \\
- \\
- \\
- \\
d \\
-\end{array}$ & $\begin{array}{c}\Lambda_{H} \\
\mu_{h} \\
\lambda_{h} \\
- \\
(1-\sigma) \\
- \\
\delta \\
- \\
\end{array}$ & $\begin{array}{c}\mu_{h} N_{h} \\
\mu_{h} \\
\lambda_{h} \\
\gamma_{h}^{*} \\
- \\
- \\
- \\
-\end{array}$ & $\begin{array}{c}\alpha_{H} N_{H} \\
\mu_{H} \\
\lambda_{H} \\
\gamma_{H P}+f_{H S} \gamma_{H D} \\
p_{2}+p_{1} p_{4}{ }^{* *} \\
\delta_{H L} \\
\mu_{K} \\
\rho_{H C} \\
\end{array}$ \\
\hline $\begin{array}{c}\text { treatment rate }(1 \mathrm{st}) \\
\text { treatment rate }(2 \mathrm{nd}) \\
\text { treatment rate }(\mathrm{PKDL}) \\
\text { probability of non-private treatment } \\
\text { (1st) treatment-induced mortality } \\
(2 \mathrm{nd}) \text { treatment-induced mortality }\end{array}$ & $\begin{array}{c}\gamma_{H} \\
- \\
\eta_{H} \\
p \\
- \\
-\end{array}$ & $\begin{array}{l}- \\
- \\
- \\
- \\
-\end{array}$ & $\begin{array}{c}\alpha_{1} \\
- \\
\beta^{\dagger} \\
- \\
- \\
-\end{array}$ & $\begin{array}{c}\alpha_{h}^{*} \\
- \\
- \\
p \\
- \\
-\end{array}$ & $\begin{array}{c}\tau_{1} \\
\tau_{2} \\
\tau_{3} \\
- \\
\mu_{T 1} \\
\mu_{T 2}\end{array}$ \\
\hline
\end{tabular}

*=Erlang distributed

**=either with or without second treatment and conditional upon survival

$\dagger=$ spontaneous PKDL recovery is given at a rate $\alpha_{2}$

$\ddagger=c$ is the vectorial capacity; see Section 2.3 for this derivation 


\subsection{Infection in dogs}

Some models take a standard SI (Mejhed et al., 2009), SEI (Salah et al., 1994; Reithinger et al., 2004) or SEIR (Ribas et al., 2013) compartmental progression for dogs, however the first model of VL in dogs (Dye, 1988) takes the approach where some dogs have an innate resistance which is fixed at birth. These dogs, which will be referred to here as "never-infectious" (to use terminology coined later by Courtenay et al. (2002b)), play no role in the transmission cycle (see Figure 8). This approach is also taken by Dye (1996) and Palatnik-de Sousa et al. (2004).

Figure 8: Model of VL progression in dogs used by Dye (1988) with $S_{D}, E_{D}, I_{D}$ being the susceptible, latent and infectious dogs respectively and the "never-infectious" population is denoted here by $R_{D}$.

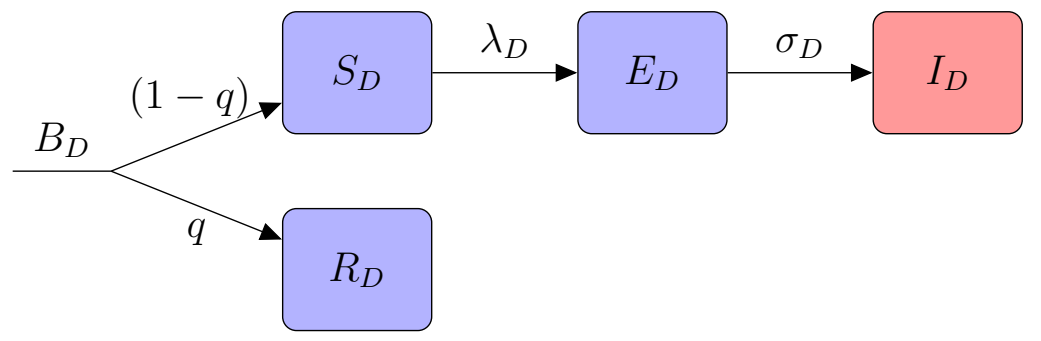

Hasibeder et al. (1992) assume a very similar progress to that of Dye (1988), however the model is subtly different by assuming that never-infectious dogs can become seropositive, yet remain non-infective to sandflies. These dogs may self-cure unlike their infective counterparts, returning to their original status of uninfected and never-infectious. This underlying model structure makes no difference to the disease dynamics compared to Dye's original model, however including this extra infected dog class may help to link seroprevalence data better to the model. This model structure is also used later by Courtenay et al. (2002b).

As with human infection, infected dogs can exhibit a range of clinical signs from completely asymptomatic to severe, fatal infection. Current diagnostics through sera or blood eluates are able to detect latent and asymptomatic infections (though with reduced sensitivity compared to infectious dogs (Courtenay et al., 2002b)). Current tests cannot reliably identify infectious dogs, though both the anti-parasite IgG antibody level and the parasite load have been shown to be highly correlated with probability of infection in sandflies during xenodiagnostic studies (Courtenay et al., 2002b, 2014). 
Figure 9: Model of VL progression in dogs used by Hasibeder et al. (1992) with "neverinfectious" population show as the bottom line. All dogs shown in the green circle are infected and assumed seropositive, however only dogs in the red box, $I_{D}$, are infective to sandflies.

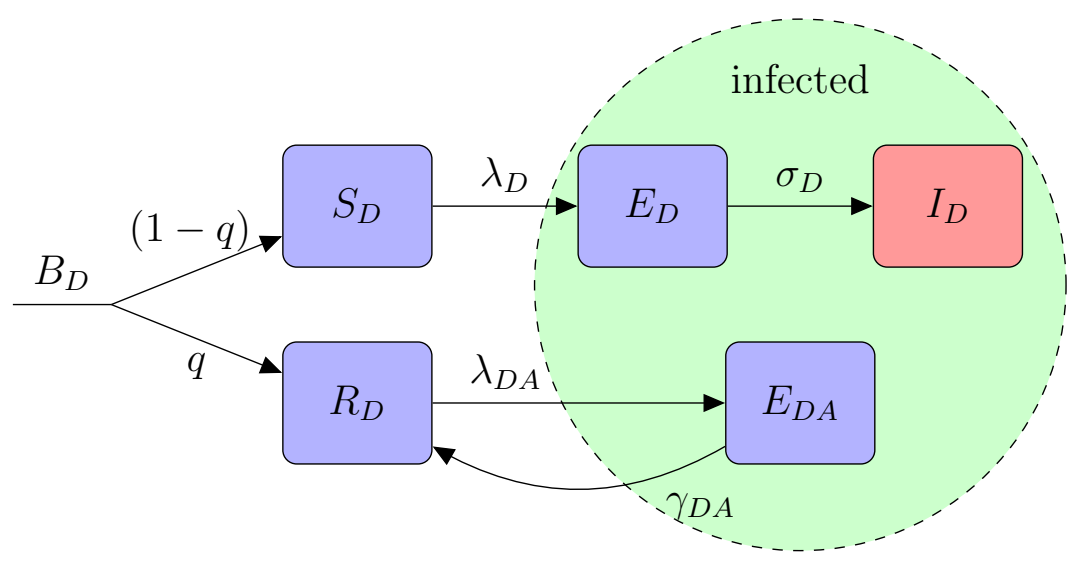

The most complex structure for ZVL is given by Costa et al. (2013a) in their model which not only accounts for possible infective asymptomatic dogs but also for imperfect screening due to diagnostic tools via the use of extra compartments (see Figure 10). This modelling approach is more sophisticated than its predecessors, with model equations corresponding to this progression allow for delay between diagnosis and culling (discussed in Section 4). 
Figure 10: Model of VL progression in dogs used by Costa et al. (2013a). Here all dogs are born susceptible but only some will develop symptoms after becoming infected and passing through the incubation period. Dogs are screened at a rate $r$ with sensitivity $d$, and correctly categorised as diagnosed with symptomatic and asymptomatic infection, $D_{S}, D_{A}$, respectively. Due to imperfect specificity $d_{Z}$ of diagnostic tools, some non-infected dogs are incorrectly diagnosed $D_{Z}$. All dogs within the green ellipse test seropositive. All red boxes correspond to infective dogs, although asymptomatic dogs are around 3-fold less infective to sandflies than those which are symptomatic.

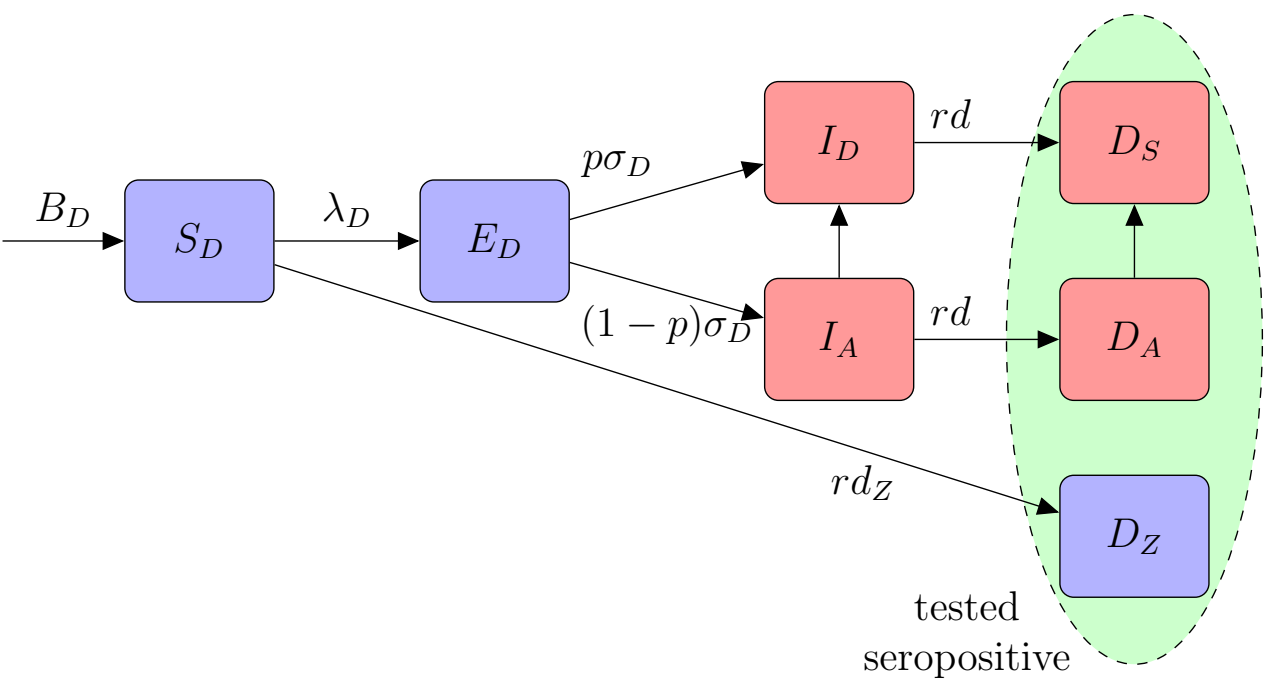


Table 2: Parameters associated with VL progression in dogs and their notation here and across modelling papers. All rates are per capita unless stated otherwise.

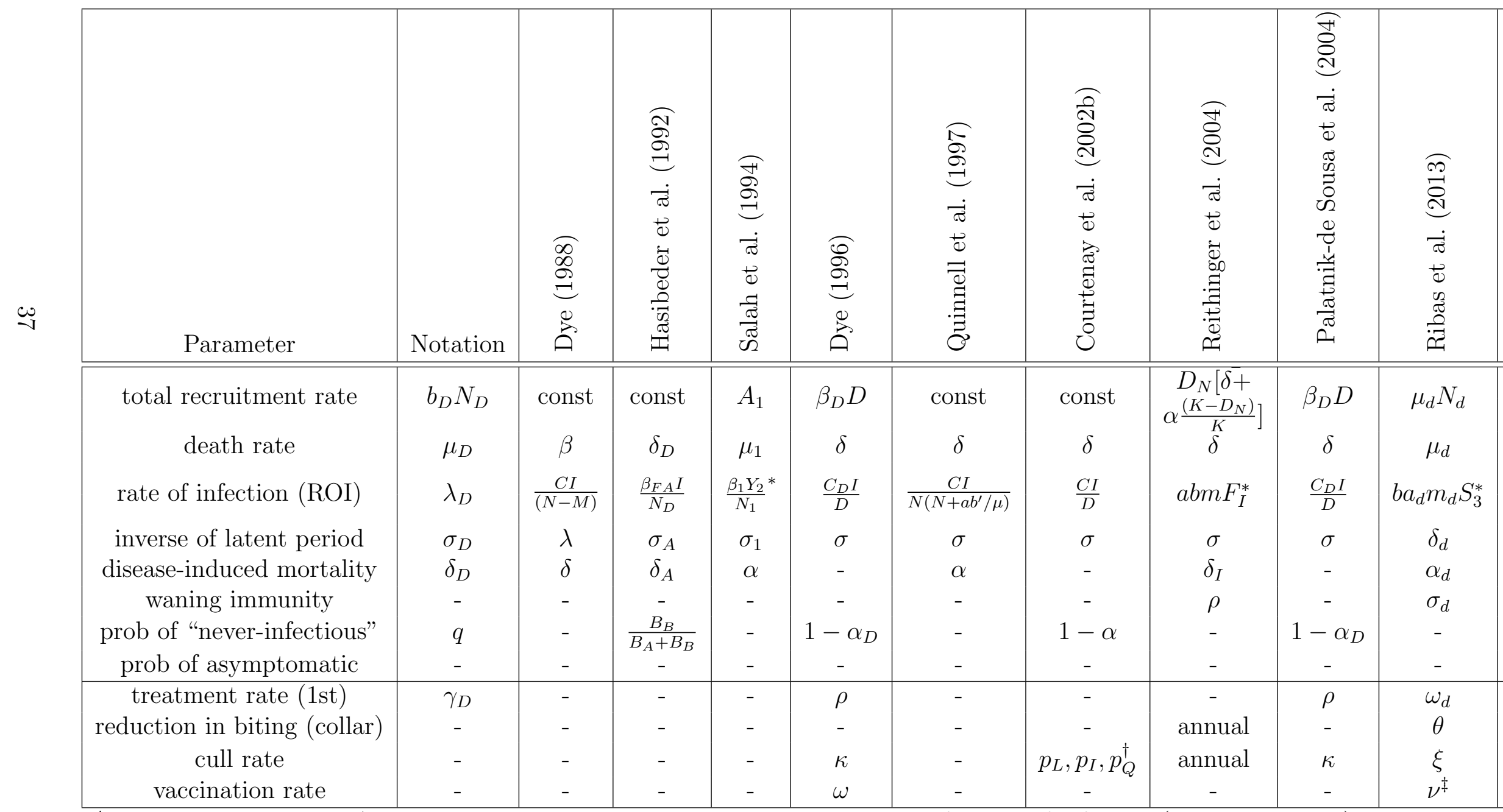

$*=$ host-vector model. All others are based on vectorial capacity assumption for rate of infection (host-only model)

$\dagger=$ annual cull of seropositive: latent, infectious and non-infectious dogs respectively

$\ddagger=$ transmission blocking vaccine 


\subsection{The role of sandflies}

Sandflies are key in the transmission cycle but little is known about some important factors. In particular with models of vector-borne disease, it is necessary to be able to estimate certain parameters in order to mechanistically model the role of the vector as part of the transmission cycle. These factors include vector density, life expectancy and relative biting rates on both humans and animals.

There are two different methods that are typically used to model hostsandfly disease dynamics, both based on the Ross-Macdonald model (Ross, 1916; Macdonald, 1957), with the second method being a simplification of the first. As is standard within vector-borne disease modelling for the first type of Ross-Macdonald model, both host and vector equations are explicitly given. Several VL models (see Table 3) use this explicit formulation with vector equations described by the ODEs:

$$
\begin{aligned}
\frac{\mathrm{d} S_{V}}{\mathrm{~d} t} & =B_{V}-\lambda_{V} S_{V}-\mu_{V} S_{V} \\
\frac{\mathrm{d} E_{V}}{\mathrm{~d} t} & =\lambda_{V} S_{V}-\sigma_{V} E_{V}-\mu_{V} E_{V} \\
\frac{\mathrm{d} I_{V}}{\mathrm{~d} t} & =\sigma_{V} E_{V}-\mu_{V} I_{V}
\end{aligned}
$$

which use an exponentially distributed extrinsic incubation period (EIP) with mean duration $1 / \sigma_{V}$. Alternatively the delay ODEs:

$$
\begin{aligned}
\frac{\mathrm{d} S_{V}}{\mathrm{~d} t} & =B_{V}-\lambda_{V} S_{V}-\mu_{V} S_{V} \\
\frac{\mathrm{d} E_{V}}{\mathrm{~d} t} & =\lambda_{V} S_{V}-\hat{\lambda}_{V} \hat{S}_{V} \exp \left(-\mu_{V} \tau\right)-\mu_{V} E_{V} \\
\frac{\mathrm{d} I_{V}}{\mathrm{~d} t} & =\hat{\lambda}_{V} \hat{S}_{V} \exp \left(-\mu_{V} \tau\right)-\mu_{V} I_{V}
\end{aligned}
$$

can be used where the caret denotes the variable evaluated $\tau$ days ago, i.e. $\hat{S}_{V}=S_{V}(t-\tau)$. This formulation has a fixed duration EIP of $\tau$. In both cases the rate of infection (ROI), often referred to as the force of infection, towards vectors takes a similar form to:

$$
\lambda_{V}=\alpha p_{V} \frac{\left(I_{H}+P_{H}\right)}{N_{H}}
$$


for $L$. donovani in human populations where $\alpha$ is the sandfly biting rate and $p_{V}$ is the infectivity towards the sandfly. With this form of the ROI towards vectors, both symptomatic cases of VL and PKDL are assumed to contribute equally to infection in sandflies. Parameter notation associated with vectors is found in Table 3.

In the case where PKDL does not occur (L. infantum) or for populations of dogs (or other animals) the ROI is simply:

$$
\lambda_{V}=\alpha p_{V} \frac{I_{H}}{N_{H}}
$$

Other additions used by Stauch et al. (2011, 2012, 2014), including infective asymptomatics and varying infectivity of HIV-positive individuals are incorporated in a similar fashion but are not written here to retain a more simple form. Amendments that can be made to consider the effect of additional animal populations are discussed later in Section 2.3.3.

The rates of infection for a "typical" vector-borne disease are usually given in the form:

$$
\begin{aligned}
\lambda_{H} & =\alpha p_{H} \frac{I_{V}}{N_{H}} \\
\lambda_{V} & =\alpha p_{V} \frac{I_{H}}{N_{H}}
\end{aligned}
$$

towards hosts and vectors respectively for a system with just one host involved in the transmission cycle. This formulation for $\lambda_{H}$ arises through an infected vector, $I_{V}$, picking a single host out of $N_{H}$ available hosts and biting at a rate $\alpha$. The probability of the host being infected through a single infectious bite is $p_{H}$. The term $\lambda_{V}$ is similar, with a single susceptible vector picking an infected host from the total host population with probability $I_{H} / N_{H}$ at a rate $\alpha$ and the probability of infection for the vector is $p_{V}$ per bite on an infected host.

However sometimes the ROI towards hosts and vectors is given by:

$$
\begin{aligned}
\lambda_{H} & =\beta_{H} \frac{I_{V}}{N_{V}} \\
\lambda_{V} & =\beta_{V} \frac{I_{H}}{N_{H}}
\end{aligned}
$$

with the denominator in the host equation being $N_{V}$ instead of $N_{H}$. This form is used by Mubayi et al. (2010) in their host-vector model of VL on the 
ISC and is one of the original variants of the Ross-Macdonald model (Smith et al., 2012). Despite the apparent difference in formulation, this form is used if host and vector populations are assumed to be constant, and so if $\beta_{H}=\alpha p_{H} N_{V} / N_{H}$ and $\beta_{V}=\alpha p_{V}$, the systems (6) and (7) are equivalent.

The second type of model, used particularly in those articles pertaining to canine VL, uses an approximation method to implicitly include vectors without the need for separate vector equations. Using the fact that sandfly life expectancy is vastly smaller than that of humans or even dogs $\left(\mu_{V}>>\right.$ $\left.\mu_{H}, \mu_{D}\right)$ it is possible to separate the vector and host timescales with the vectors equilibrating much faster than hosts (Williams and Dye, 1997). This method takes one of the two forms above (either (2) or (3)) to find the equilibrium number of infectious sandflies, and therefore a quasiequilibrium (or pseudoequilibrium) approximation of the host infection dynamics.

The resulting approximation for the infectious vectors is:

$$
I_{V}^{*} \approx N_{V} \frac{\lambda_{V}}{\left(\lambda_{V}+\mu_{V}\right)} \times \mathbb{P}(\text { survive EIP })
$$

where the star denotes equilibrium, $\lambda_{V}$ is as before (such as (4) or (5)) and the probability of surviving the EIP is given by:

$\mathbb{P}($ survive EIP $)=\left\{\begin{array}{cl}1 & \text { no incubation period } \\ \exp \left(-\mu_{V} \tau\right) & \text { fixed duration EIP } \\ \frac{\sigma_{V}}{\sigma_{V}+\mu_{V}} & \text { exponentially distributed EIP } \\ \left(\frac{n \sigma_{V}}{n \sigma_{V}+\mu_{V}}\right)^{n} & \text { gamma distributed EIP where shape parameter } n\end{array}\right.$

under various assumptions with the same mean duration used in the VL modelling literature.

This may then be substituted into the ROI towards the host population(s) by using:

$$
\lambda_{H}=\alpha p_{H} \frac{I_{V}^{*}}{N_{H}}
$$

and so only host equations need to be explicitly written down.

In the case of VL, this approximation is almost always taken further by considering the relative size of parameters in comparison to prevalence in 
hosts, i.e. if $\mu_{V}>>\lambda_{V}$. Under this new condition the equilibrium infection in sandflies is given by:

$$
I_{V}^{*} \approx N_{V} \frac{\lambda_{V}}{\mu_{V}} \times \mathbb{P}(\text { survive EIP })
$$

In the case of endemic L. infantum infection in dogs in Brazil, dog infection prevalence is around $19 \%$, with a sandfly biting rate of approximately once every 3 days (Reithinger et al., 2004), sandfly life expectancy of 14 days (this is an upper estimate from the huge range of 2.4 (Dye, 1996) to 16.7 days (Ribas et al., 2013)) and infectivity towards sandflies of 10.7\% (Courtenay et al., 2002b). Under this parameterisation $\mu_{V} \approx 0.0714$ and $\lambda_{V} \approx 0.0068$ and so $\mu_{V}$ is an order of magnitude larger. Other less conservative estimates for these parameters in this setting further increase the difference between these two values, making the approximation quite reasonable, however in some regions with higher host prevalence or alternate parameterisation this assumption would not be appropriate. Care must also be taken to consider the expected impact of control and seasonality on these approximations, which might make them less appropriate.

This formulation is often favoured as it can be directly related to an epidemiological measure, the vectorial capacity. The vectorial capacity is the number of new cases occurring from all the bites taken on a single infected person on one day in an otherwise naïve population. It is usually denoted by:

$$
C:=\frac{N_{V} \alpha^{2} p_{H} p_{V}}{\mu_{V} N_{H}} \times \mathbb{P}(\text { survive EIP })
$$

and so the ROI towards humans can be described in terms of the vectorial capacity:

$$
\begin{aligned}
\lambda_{H} & =\alpha p_{H} \frac{I_{V^{*}}}{N_{H}} \\
& \approx \frac{\alpha^{2} N_{V} p_{H} p_{V}}{\mu_{V} N_{H}} \times \mathbb{P}(\text { survive EIP }) \times \frac{I_{H}}{N_{H}} \\
& =C \frac{I_{H}}{N_{H}}
\end{aligned}
$$

which leads to a host-only model with the same type of frequency-dependent contact rate used in contagious disease modelling (Anderson and May, 1992). Several models of VL (Dye and Wolpert, 1988; Dye, 1988, 1996; Courtenay et al., 2002b; Quinnell et al., 1997; Palatnik-de Sousa et al., 2004; Costa et al., 2013a) utilise this vectorial capacity quasiequilibrium assumption. 
Finally, as is often the case with cost-benefit analyses, some models (Boelaert et al., 1999; Meheus et al., 2010; Lee et al., 2012) do not use a dynamic, prevalence-dependent ROI and so the role of vectors is not considered at all.

\subsubsection{The basic reproduction number}

Alongside the vectorial capacity, another epidemiology measure known as the basic reproduction number, or basic reproductive ratio, $R_{0}$, is often used when comparing disease in different regions or examining elimination. Unlike the vectorial capacity which is primarily related to the vector's capacity to transmit disease and therefore is necessarily restricted to vector-borne diseases, $R_{0}$ depends on parameters associated with all parts of the infection cycle and is widely regarded as the most useful measure across a range of diseases (Keeling and Rohani, 2008).

For contagious disease in a homogeneously mixing population, $R_{0}$ summarises the number of new cases occurring directly from a single infected individual in fully susceptible population without intervention. However for heterogeneous populations with structured mixing or multiple species the definition is slightly trickier (Rock et al., 2014). In its formal mathematical derivation, $R_{0}$ is defined using the next generation matrix approach (Diekmann et al., 1990); this computes the average number of secondary infections from a "typical" infected individual. Here a "typical" individual may be a host or a vector and the secondary infections, or the next generation, for VL will always arise in the opposite population as hosts can only infect vectors and vice versa. For basic VL models this method is not too complex.

In the case where vector EIP is assumed to be exponentially distributed (2) and there is an SEIR host progression, the next generation matrix, $K$, is calculated by writing down the matrices of transmissions, $T$, and transitions, $\Sigma$, associated with the variables $E_{H}, I_{H}, E_{V}$ and $I_{V}$ of infected and infectious hosts and vectors.

$$
T=\left(\begin{array}{cccc}
0 & 0 & 0 & \alpha p_{H} \frac{N_{H}}{N_{H}} \\
0 & 0 & 0 & 0 \\
0 & \alpha p_{V} \frac{N_{V}}{N_{H}} & 0 & 0 \\
0 & 0 & 0 & 0
\end{array}\right)
$$




$$
\Sigma=\left(\begin{array}{cccc}
-\left(\sigma_{H}+\mu_{H}\right) & 0 & 0 & 0 \\
\sigma_{H} & -\left(\gamma_{H}+\delta_{H}+\mu_{H}\right) & 0 & 0 \\
0 & 0 & -\left(\sigma_{V}+\mu_{V}\right) & 0 \\
0 & 0 & \sigma_{V} & -\mu_{V}
\end{array}\right)
$$

and the next generation matrix is given by:

$$
K=-T \Sigma^{-1}
$$

Finally $R_{0}^{N G M}$ is the largest eigenvalue of the matrix, which is the spectral radius of $K$ :

$$
R_{0}^{N G M}=\rho(K)=\sqrt{\frac{N_{V} \alpha^{2} p_{H} p_{V}}{\mu_{V} N_{H}} \frac{\sigma_{V}}{\left(\sigma_{V}+\mu_{V}\right)} \frac{\sigma_{H}}{\left(\sigma_{H}+\mu_{H}\right)} \frac{1}{\left(\mu_{H}+\gamma_{H}+\delta_{H}\right)}}
$$

For VL that includes PKDL, alternate disease progressions or multiple hosts this methodology can also be used. It can now be seen how $R_{0}$ and the vectorial capacity are linked:

$$
\left(R_{0}^{N G M}\right)^{2}=C \frac{\sigma_{H}}{\left(\sigma_{H}+\mu_{H}\right)} \frac{1}{\left(\mu_{H}+\gamma_{H}+\delta_{H}\right)}
$$

Confusingly, for vector-borne disease, the basic reproductive ratio is often considered to be the expected number of new cases in the host population generated by a single infected host (Smith et al., 2007). As this is two infectious generations, rather than one, this expected number of cases is the square of $R_{0}^{N G M}$ given in (17). This version of the basic reproductive ratio, called here $R_{0}^{\text {full }}$, links to a full host-to-host cycle can also be derived using the equations for the host-only model. Articles which use this version include Dye (1996) and Quinnell et al. (1997) and arguably $R_{0}^{\text {full }}$ could be considered to be a more intuitive metric as it has a relatively simple biological interpretation.

Conversion between the two versions of the basic reproductive ratio is trivial and they agree on the same critical threshold value of $R_{0}=1$, above which a disease can invade a population and below which, it cannot. Caution need only be taken when using the value of $R_{0}$ as metric to compare either disease in different settings or between different models; for example between that of Dye and Wolpert $(1988)\left(R_{0}^{\text {full }}=2.13\right)$ and Stauch et al. (2011) $\left(R_{0}^{N G M}=3.94\right.$, which gives $\left.R_{0}^{\text {full }}=15.5\right)$ who both model VL on the ISC. 


\subsubsection{Sandfly host choice}

Vector choice of host can be important to the spread of vector-borne disease. The presence of secondary hosts plays a key role in reducing the biting pressure upon the primary species, however if the secondary hosts are also able to acquire and transmit the parasite they could become a reservoir for infection. The concept of zooprophylaxis, where animals which are not part of the transmission cycle are used to deflect biting away from humans, has been suggested for other vector-borne diseases including malaria and Chagas disease (WHO, 1982; Cruz-Pacheco et al., 2012).

On the ISC where the disease is thought to be anthroponotic, it is reasonable to expect that additional animal populations have protective effects for the human population by moving the biting pressure away from humans. However the presence of animals close to the home may draw sandflies to the area, resulting in higher vector densities and more risk. For the ISC studies are conflicted about the protectiveness of cattle with some finding significant protection and but others determining an increased risk (Bern et al., 2010). For L. infantum, dogs are a known reservoir of infection and play a major role in transmission whereas chickens could have a zooprophylactic role by shifting biting pressure away from humans and effectively reducing the vector to host density.

Biting behaviour of sandflies ( $P$. argentipes on the ISC and Lu. longipalpis in the New World) seems to be primarily opportunistic, however host biomass is thought to play a role in host selection (Quinnell and Courtenay, 2009; Bern et al., 2010). In addition to relative human-animal and human-vector densities, this sandfly host preference will impact upon transmission unless sandflies are motivated solely by convenience as has been described for other vector-borne diseases (Rock et al., 2015b).

Despite this, not all modelling work integrates animal (or even multiple animal) populations. Where animals are part of the transmission cycle, the ROI towards the vector can be written:

$$
\lambda_{V}=\alpha p_{V} \frac{a\left(I_{H}+P_{H}\right)+(1-a) I_{A}}{a N_{H}+(1-a) N_{A}}
$$

Where $N_{A}$ is the number of animals, $I_{A}$ is the number of infected animals, and $a$ is the relative propensity to feed on humans rather than animals. Stauch et al. (2011, 2012, 2014) later on discount animal reservoirs by setting $a=1$, but ELmojtaba et al. (2010a) use this type of ROI in their model 
analysis to examine control via this reservoir (see Section 4.4). Even if animals play no role, the same formulation can be used with $a<1$ but $I_{A}=0$ in order to model the reduction in biting due to presence of animals (i.e. zooprophylatic effects).

\subsubsection{Seasonality of sandflies}

Seasonality of sandfly populations is considered to be substantial however it is not included in any models of VL on the ISC and only a handful elsewhere (Salah et al., 1994; Mejhed et al., 2009; Williams and Dye, 1997; Quinnell et al., 1997). Some non-modelling papers have sought to quantify or qualitatively assess seasonal aspects of sandfly emergence or biting (Singh et al., 2008; Rossi et al., 2008; Picado et al., 2010a) and a paper on cutaneous leishmaniasis has incorporated detailed information about sandfly dynamics into a transmission model (Bacaër and Guernaoui, 2006). Salah et al. (1994) alter the host-vector transmission terms by means of a Boolean function so that transmission only occurs during the warmer 5 months of the year and Mejhed et al. (2009) use seasonal data of sandfly population sizes from Morocco to parameterise the model over the course of a year. The others, Quinnell et al. (1997) and Williams and Dye (1997), describe how a host-only model of VL may be adapted to include seasonality by using fluctuating vectorial capacity.

In reality there is a complex relationship between vector mortality/emergence, EIP, human biting rate and temperature (as discussed by Hartemink et al. (2011)), however to facilitate the modelling process, seasonality is usually associated with either change in vector emergence rate, or instead by varying the transmission rate but keeping constant vector population size.

Figure 11 shows how using a sinusoidal forcing function of the form $\left(1+\alpha_{1} \cos (2 \pi t)\right)$ on either the birth rate or the ROI affects the disease about equilibrium for both host and vector populations. Although the two types of forcing are fundamentally different the change in the resultant dynamics and ROI between the two is slight (see Figure 12b); as would be expected, forcing vector and host ROI shifts the peak of infection a little earlier than forcing vector births as there is a lag between the influx of newly emerged vectors and them biting and becoming infected. The most noticeable difference occurs within the vector dynamics; adjusting the ROI in this way has no impact upon the vector population size, however changing the birth rate does (see Figure 11c). Consequently, whilst very similar dynamics in the host population are seen in these two cases, the prevalence measured in the 
vector population across the year shows a marked difference, reaching very high prevalence just before the coldest part of the year using forced births.

Under a forced birth rate $R_{0}^{N G M}$ will be slightly underestimated (by $\alpha_{1} / 8 \%$ where $\alpha_{1}$ is the amplitude of forcing in the function given above) (c.f. Bacaër, 2007). Williams and Dye (1997) describe how this is related to changes in incidence and how it will affect the net reproductive ratio and its thresholds. In the typical case where per capita host death and recovery rates are taken to be constant and forcing is on the vectorial capacity, then the arithmetic mean dictates the overall dynamics and so the critical threshold of $R_{0}=1$ still holds. Additionally, in the case of vector-borne disease including ZVL, knowledge of varying incidence (which can be measured in the field) in the host population can be used to calculate $R_{0}$ as average incidence corresponds to average vectorial capacity.

Seasonality in ROI can be critical in interpretation of surveillance data and in model predictions of intervention outcomes. Repeated data on sandfly population size and infection prevalence is required to fit models that include seasonality. The mechanistic functions suggested above could be combined, and other mechanisms included. If seasonality is due to environmental variables, such as rainfall and temperature, then there is no reason for them to follow particular patterns, which further complicates data requirements and ability to forecast accurately.

Other options for season forcing include Boolean transmission where transmission occurs only at certain times of the year and not in others, for example the 5 warmest months out of the year (after Salah et al., 1994). Figure 12 demonstrates how altering the biting function in this way compares to the previous sinusoidal biting function.

There is clearly a need for regular sampling to estimate vector parameters across the seasons to better inform fluctuating transmission from sandflies. Sampling frequency needs to be of the same order of magnitude as the generation time. Inadequate sampling frequency, e.g. annual, cannot discern sandfly dynamics and could even give a distorted picture of average sandfly prevalence or rate of infection towards host populations. Mejhed et al. (2009) indicate that May/June and October are the two times of the year when there is the highest values for $R_{0}$ and so highest levels of transmission in Marrakech, Morocco. Similarly, on the ISC, Picado et al. (2010a) argue that IRS should be carried out in Feb/March and August/September based on seasonal patterns observed in their sandfly density studies in India and Nepal. However a study in Bihar, India which examined sandfly infection as well as density 

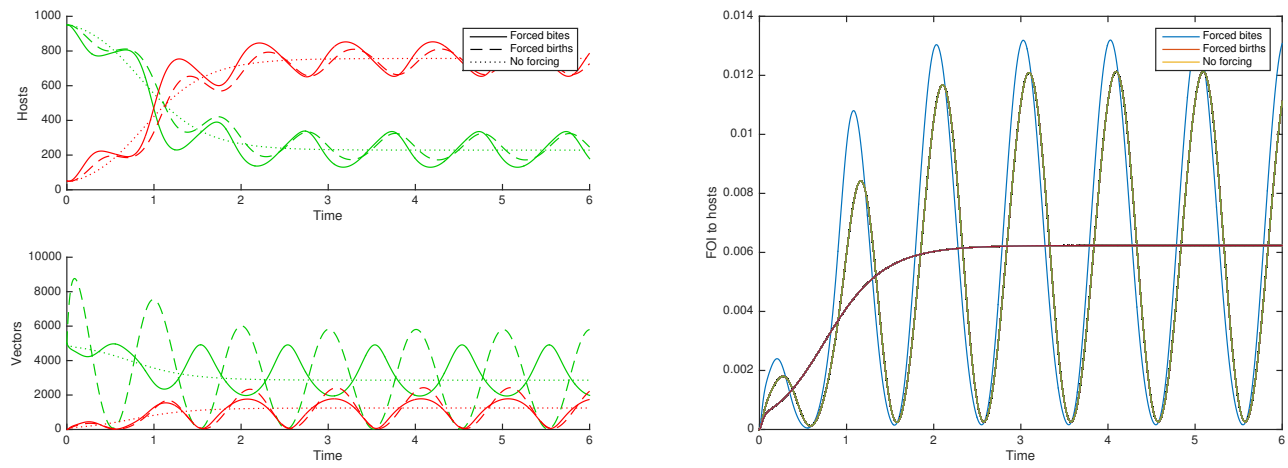

(a) Host and vector infection dynamics

(b) ROI towards humans
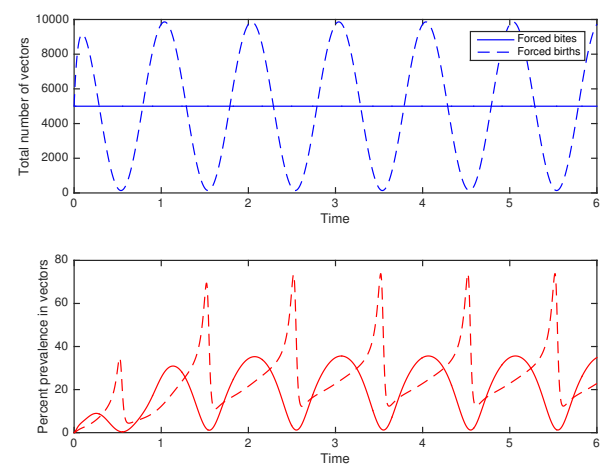

(c) ROI towards hosts

Figure 11: Simulations using no forcing and sinusoidal forcing on the birth rate and ROI respectively. (a) shows the expected change in level for susceptible (green) and infected (red) host and vectors for a SEIR host/SEI vector model. (b) shows how the ROI will change under the three assumptions and (c) compares the population size and percent prevalence of infection in the vector population under forced births and forced ROI. 

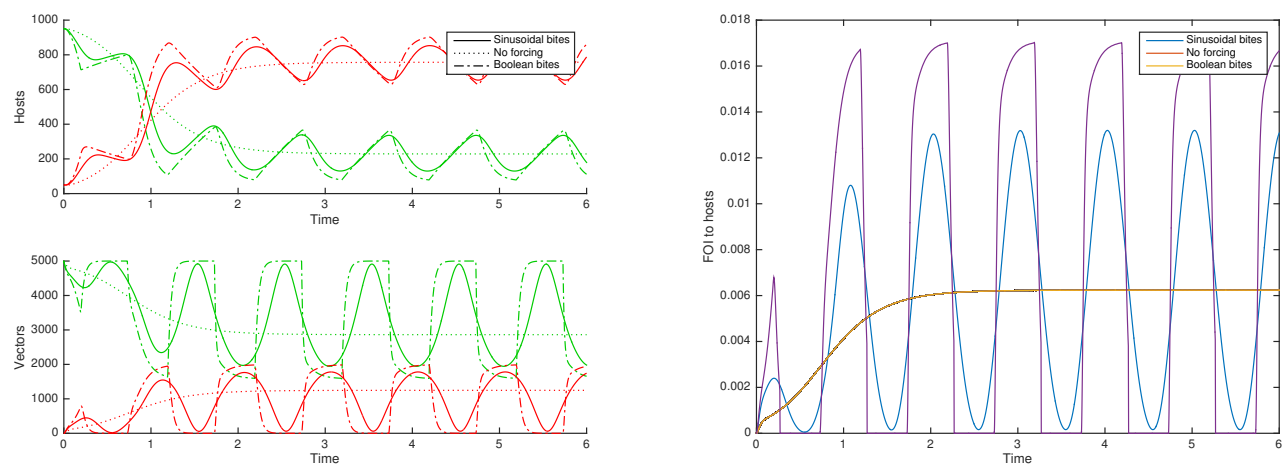

(a) Host and vector infection dynamics

(b) ROI towards hosts

Figure 12: Simulations using no forcing and sinusoidal/boolean forcing on the ROI respectively. (a) shows the expected change in level for susceptible (green) and infected (red) host and vectors for a SEIR host/SEI vector model. (b) shows how the ROI will change under the three assumptions.

found that whilst abundance was highest during August/September, prevalence in sandflies was greatest in November/December (Tiwary et al., 2013). Such results warrant further investigation into the seasonality in all endemic regions, especially when planning the timing of vector interventions such as IRS. 
Table 3: Parameters associated with VL in vectors and their notation here and across modelling papers where they are explicitly included. All rates are per capita unless stated otherwise.

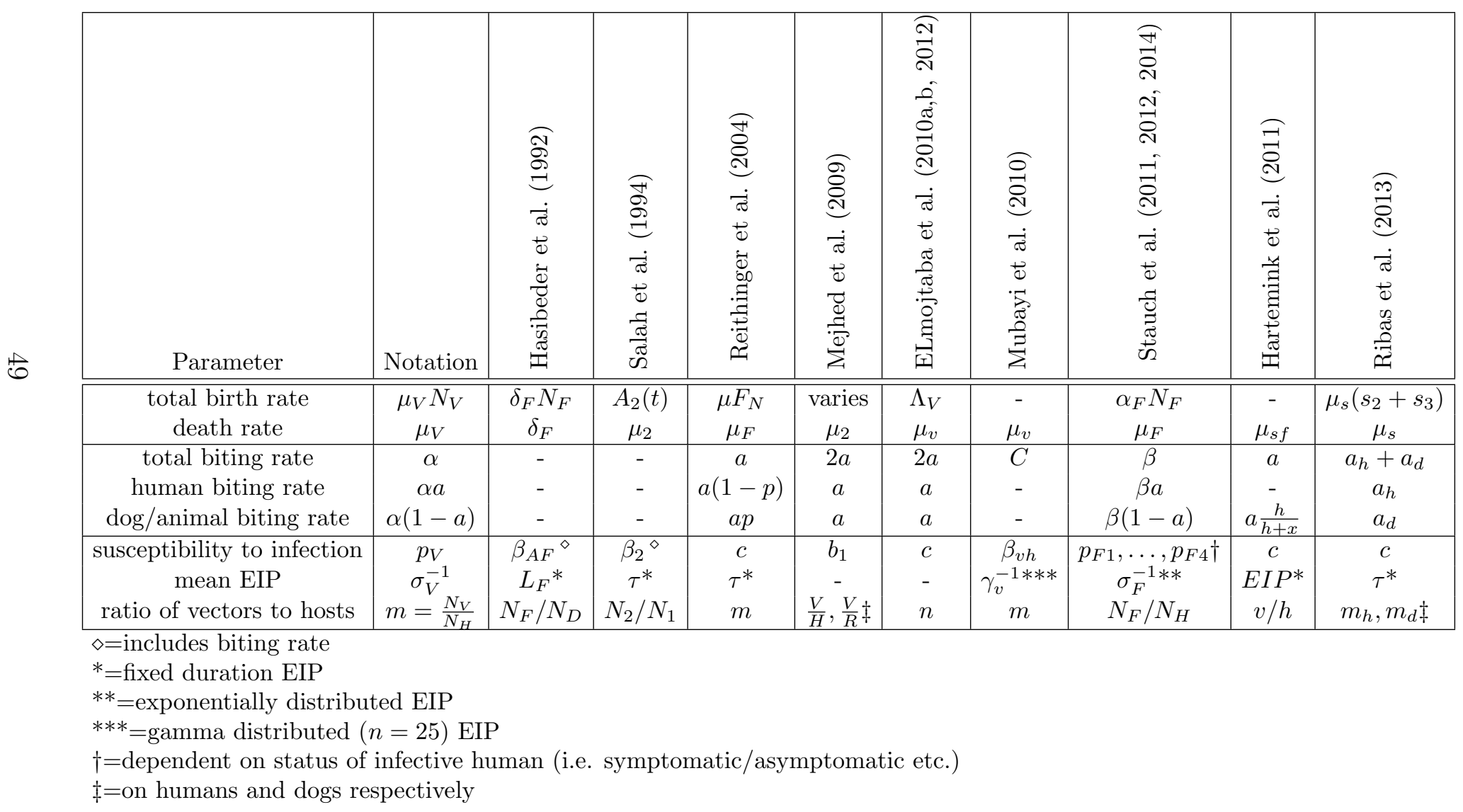




\section{Data-Model Interactions}

\subsection{Current data and initial models}

In any model, individuals have to have a defined infection state. For compartmental models, these states are mutually exclusive, for example, susceptible (uninfected) and infected. There are two general approaches to fitting models to data. First, individuals are unambiguously assigned to one compartment/state on the basis of diagnostic tests, and the numbers of individuals in each diagnostic category is compared to model prediction (e.g. Stauch et al., 2011, 2012, 2014; Chapman et al., 2015). Alternatively, the model states can be related to population data through diagnostic characteristics, i.e. the model can predict the prevalence of positives (true and false) for a given infection prevalence. The latter approach is most often related to age groups (e.g. Rodriguez-Barraquer et al., 2014). In either case, but especially the former, the information in the data, and therefore the accuracy of parameter estimates, is greatly enhanced if the data are longitudinal, i.e. multiple measurements from the same individuals through time (e.g. Chapman et al., 2015). Time series data arise from repeated measurements in the same population, but not necessarily the same individuals; an example is data relating to cases in a defined population over time. Age-related data are more informative than simple prevalence data, as age measures duration of exposure, and can serve as a proxy for time. Having defined the states in the model that can be related to data, the states have to be defined in terms of their duration and their infectiousness. These data can be inferred during a fitting process, or, better, estimated independently from research data. As has already been discussed, a major complication with Leishmania infection is that precise, gold standard diagnostic does not exist. In a modelling context, a diagnostic is good if it is accurate and matches well with compartments required in the model. Of course, modellers will frequently choose compartments that match with diagnostics, and Stauch et al. (2011, $2012,2014)$ is an one such example. In contrast, diagnostics and vector mea-

surement are more advanced in malaria, which has inspired a plethora of modelling approaches (Smith et al., 2014).

The dynamics of the vector population directly impact the observed VL transmission dynamics. However, several of the parameters associated with the vector are hard to ascertain and consequently there are great uncertainties in the parameterisation of models, in particular with those which explicitly model vector populations. The density of sandflies and their abun- 
dance relative to both human and animal hosts are not only difficult to determine but are known to vary greatly between different regions, for example, see Hartemink et al. (2011) who used geographic sticky trap data to estimate densities and map $R_{0}$ in France. Likewise the seasonal fluctuations of sandfly populations are hard to pin down and so are not featured in many transmission models of VL at present. Even something as fundamental as sandfly life expectancy has large confidence intervals and whilst models typically use an estimate of 2 weeks, they range from 2 days to an unlikely 2 months.

One way to make progress without this information is to create host-only models which use the vectorial capacity, $C$, to infer the host-vector component of transmission. Although this is useful for initial models, and is consistent and efficient from a modelling view point, the value of $C$ is still reliant on estimating several vector parameters, and the usual assumption that the quasiequilibrium assumption is valid. We are also missing accurate assessment of the infectiousness of different host classes to sandflies, and again, in order to make progress, simplifying assumptions have to be made, for example, Stauch et al. (2011, 2012, 2014) use $p_{V}=1$ for the probability of a sandfly acquiring infection from an infected human. Of course, without information on sandfly biting rates, this assumption doesn't matter, as doubling the sandfly population and halving $p_{V}$ will give the same results. Parameter combinations such as $R_{0}$ and $C$ summarise both the model and unknown data into a single number.

A feature of simple models of infectious disease is that apart from the infection state, and perhaps age, all individuals are considered as equal representatives of the average individual, and all are equally likely to transmit to each other. However, there will be considerable heterogeneities in terms of, for example, response to infection, and exposure to vectors. If this heterogeneity is great, then the concept of an average might be wrong (e.g. Dye (1986); Smith et al. (2014)). The consequence of mistakenly believing individuals are all essentially equal is usually to under-estimate the control effort required (i.e. to underestimate $R_{0}$ ), as there are sections of the host-vectorparasite population combinations that are transmitting at a much higher rate than the rest. For Leishmania in particular, there is sufficient field evidence to suggest that rates of transmission and disease vary significantly through both time (Dye and Wolpert, 1988) and space (Bern et al., 2007; Woolhouse et al., 1997), and between individuals (Courtenay et al., 2014) so that there is unlikely to be a good "average" situation. But without sufficient 
data to understand the important spatio-temporal scales, the correct scale of appropriate spatio-temporal studies is unknown; for example, is annual assessment of a village sufficient, or should studies be based on monthly assessment of households in at least five villages? For malaria, spatio-temporal hotspots have been identified at the household-month level (Bejon et al., 2014). Broad estimates may still help in planning widespread interventions, but more detailed information could help target interventions in key foci at optimal times.

As well as space and time, host-parasite-vector populations can differ by genetic, socio-economic and environmental variables. The importance of dimensions is likely to be different at different times and places - for example during an epidemic, the socio-economic factors might change. Unless the correct scales are known, the danger is that any one study gives only a partial picture, and piecing the studies together is fraught with difficulty due to, for example, difference between epidemiology stages and variation in diagnostics. Additionally, highly variable diagnostics are also going to potentially mask important heterogeneities, i.e. the noise will swamp the signal.

\subsection{Future data and alternative sources}

Given the relative paucity of data that can be used to develop accurate models, can we identify alternative sources of data, and define from current models and understanding, what data ought to be collected?

Working with animal models of infection and disease has the considerable benefit that it is possible to conduct experiments (e.g. deliberate infection), but the disadvantage that the results might not transfer directly to humans. Studying canine, zoonotic VL has the advantage that parameter estimates can directly inform models relevant to zoonotic human disease, as well as, in the absence of direct data, informing anthroponotic transmission models. Whilst the quantitative parameter estimates are unlikely to be directly transferable, the processes might be directly parallel to humans. For example, infection rates of sandflies from xenodiagnosis experiments in dogs have been shown to correspond to a threshold density of Leishmania parasites in skin snips taken from ears (Courtenay et al., 2014); so whilst the numerical values of transmission probabilities are for dog-sandfly interactions, it is likely that same biological process occurs in human-sandfly interactions. It is possible to perform xenodiagnosis directly in people, and there are currently attempts to conduct such experiments (Miller et al., 2014). Similarly, 
it is possible that the natural history of disease is related to the size of the inoculating dose of infection (Bern et al., 2010).

Longitudinal and time series data will be critical to future model development. The transmission dynamics at the population level (i.e. the processes that determine the public health impact of VL), are derived from the processes within individuals: infection, immunity, pathogenesis and infectiousness. We need to know the natural history of infection, and particularly the performance of current and future diagnostics, over long periods of time. This can come from cohort studies, but also from accurate case series in defined locations, perhaps supplemented with cross-sectional data from the same populations. To our knowledge, there is no dataset available from the ISC or elsewhere which has longitudinal measurements on humans, and repeated measurements on vectors.

\section{Modelling Interventions}

\subsection{Humans}

\subsubsection{Diagnostics}

Diagnostic tools are a key part of the targeted control strategy for VL in order to accurately diagnose and treat individuals, and to monitor incidence and prevalence in the population. In addition to these primary uses, diagnostic tests for VL can also aid in developing and parameterising mathematical models of disease; enabling individuals to be placed into one of the mutually exclusive compartments.

For VL in humans some of the main diagnostic tools which can be used to screen people are PCR, ELISA, DAT, LST and rK39 (a rapid diagnostic test) as described in Section 1.1.2. Whilst some study sites may use a combination of tools to determine presence of the parasite, antigens or antibodies, often just a single test will be used to make a diagnosis. Given that mortality of the disease is associated with clinical symptoms, only those displaying outwards signs of infection are usually tested and subsequently treated.

Stauch et al. (2011, 2012, 2014) use three different diagnostics (PCR/LST/DAT) to partition the human population under their model, however the model does not account for imperfect tests. Furthermore, whilst the accuracy of tests such as PCR and DAT has been well analysed for detecting KA, they have unknown sensitivity and specificity for asymptomatic infection.

Using a cost-effectiveness analysis Boelaert et al. (1999) compared four alternate diagnostic strategies to find the most effective (most deaths averted 
compared to no intervention) and the most cost-effective (the lowest cost per death averted). The strategies are outlined in Table 4 and were based on treatment using antimonials. The authors found that for expected baseline estimates for prior probability in suspected clinical cases (40\%), that strategy A was most effective and B was worst. For lower probabilities of infection the most effective strategy changed with A causing more deaths (through possible treatment toxicity) than it averted. This result has important implications: as prevalence reduces, moving forward to elimination, it suggests that strategies may have to change as the prevalence of disease in the population decreases. The most cost-effective strategy was B at a cost of US $\$ 448$ per death averted. This analysis was published in 1999 and was based on the cost and accuracy and of drugs and diagnostics at the time. With new diagnostic developments, lower incidences and newer drugs, the available strategies and their (cost-)effectiveness need to be continually reevaluated.

Table 4: Competing diagnostic and treatment strategies analysed by Boelaert et al. (1999) in the Sudan.

\begin{tabular}{|c|l|}
\hline Strategy & Description \\
\hline \hline A & Treat all clinical suspects \\
\hline B & $\begin{array}{l}\text { Perform parasitological diagnosis } \\
\text { on clinical suspects and treat if } \\
\text { positive }\end{array}$ \\
\hline C & $\begin{array}{l}\text { Perform DAT test on clinical sus- } \\
\text { pects and treat if high titre. If } \\
\text { borderline DAT results then par- } \\
\text { asitological diagnosis and treat- } \\
\text { ment if positive. }\end{array}$ \\
\hline D & $\begin{array}{l}\text { Perform DAT test on clinical sus- } \\
\text { pects and treat if high titre }\end{array}$ \\
\hline
\end{tabular}

Recent work (Medley et al., In Press) highlights the importance of early diagnosis and treatment of KA patients, if they are responsible for the majority of transmission (i.e. if asymptomatics are not responsible for maintaining transmission). It also potentially explains why current control programmes in the ISC have been successful despite the apparent ineffectiveness of vector 
control (Coleman et al., 2015). Results from this model suggest that a diagnostic test with only $50 \%$ sensitivity might result in a considerable reduction (halving) of $R_{0}$ and offer the potential for elimination. However, low specificity of a diagnostic test (i.e. the false positive rate) would prevent it being applied to individuals with non-specific clinical symptoms. These results suggest that a diagnostic test with high specificity, even at the expense of relatively low sensitivity, is required for wide spread intervention. As elimination is approached, false positives will always become more significant, and the specificity of tests more important. However, most consideration of test characteristics during development concentrates on sensitivity.

\subsubsection{Treatment}

Treatment of KA plays an important part in control programmes, primarily as the disease is usually fatal without it. However, as treatment reduces the number of KA patients, it will also reduce the ROI towards the sandfly. The shorter the timescale between onset of KA and successful treatment, the smaller the infectious pool of people will be. However, this effect may be small if asymptomatics are important in transmission (Stauch et al., 2011).

For the Sudan, ELmojtaba et al. (2010a) examined the impact of first-line treatment for KA; analysis varied both treatment rate and effectiveness of preventing PKDL (i.e. $f$ ). With high treatment alone there is a reduction in symptomatic VL cases but since $(1-f)$ of these treatments produce a PKDL case, infection rates were still high. Increasing both treatment and $f$ was unsurprisingly more effective. Unfortunately the PKDL treatment rate was not varied to simulate extra treatment efforts with this class of patients.

The model of Stauch et al. $(2011,2012,2014)$ includes three treatment types: first line treatment of VL (at a rate $\tau_{1}$ ), second line treatment of VL following first line treatment failure (at a rate $\tau_{2}$ ) and finally PKDL treatment (at a rate $\tau_{3}$ ). First line treatment failure occurs with probability $p_{1}=5 \%$ and PKDL follows after treatment and remission with probability $p_{2}=3 \%$ from successful first line treatments and $p_{4}=3 \%$ from second line treatments (all conditional on surviving treatment with probability $\left(1-f_{T}\right) \approx 0.95$ ). Second line treatment is always assumed successful upon survival, in that individuals appear recovered at least for a time before PKDL. The authors found that whilst treatment was clearly necessary from an individual KA patient's perspective and did decrease the prevalence of KA, increasing effectiveness or time to treatment made a negligible different to the underlying disease dynamics. If this is the case and the objective is to minimise occur- 
rence of KA, then the benefit will only be gained whilst treatment continues at a high level; once the treatment returns to the original level, the prevalence will also bounce back. Stauch et al concluded that the limited effect of treatment alone on the incidence of KA was primarily due to infective asymptomatics. In fact, under their parameterisation, there would still be over $15 \mathrm{KA}$ cases per 10000 in this endemic setting. The difference between the conclusion of Stauch et al and that of Medley et al is entirely due to assumptions about the relative infectiousness of symptomatic and asymptomatic cases. The apparent clustering of KA cases and recent success of $\mathrm{KA}$ treatment in reducing VL in ISC are both evidence for KA cases being the predominant source of infection to sandflies. On the other hand, asymptomatic cases are numerous and may be significantly infectious. Only field data can resolve this difference.

A cost-effectiveness analysis by Meheus et al. (2010) compared the use of different treatment options (either currently available or in Stage III trials) on the ISC. This study differed from Boelaert et al. (1999) in geographic location, but also Meheus et al assumed patients were correctly identified prior to treatment, whereas Boelaert et al assumed all patients received the same treatment. The 10 alternate treatment strategies included a range of mono- and combination-therapies with each having associated pros and cons such as the requirement for cold-chain storage, cost, length of treatment and toxicity. The most cost-effective strategy was combination Miltefosine (MIL) and paromomycin (PM) treatment at a cost of US\$92 per death averted. The authors found that a possible future decrease in the cost of liposomal amphotericin B (L-Amb) compared to MIL might change the most costeffective strategy to combination L-Amb and PM.

\subsubsection{Human vaccine (theoretical)}

A prophylactic vaccine would reduce the human incidence by reducing the numbers of susceptibles who are able to acquire Leishmania infection, or reduce the proportion of those infected who develop disease. The former vaccine provides protection to those who have been vaccinated, but also indirect protection to the non-vaccinated as there are fewer people infected. The latter, disease preventing vaccine does not prevent the acquisition of parasites, but alters the progression so that people do not develop KA. This latter vaccine type is currently the a promising candidate for use in the near future; however asymptomatic individuals who do not develop KA may still remain infectious, so there may possibly be less indirect protection to the 


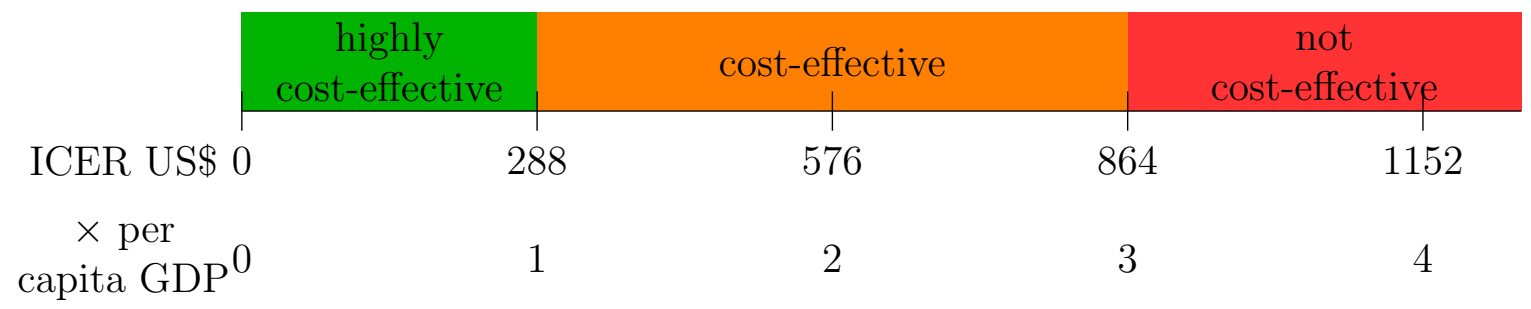

Figure 13: This diagram shows the incremental cost-effectiveness ratio (ICER) compared to the per capita GDP in Bihar. It also shows the ranges given by Lee et al. (2012) for "highly cost-effective", "cost-effective" and "not cost-effective".

non-vaccinated.

Lee et al. (2012) use a cost-effectiveness analysis to address the potential impact of a human vaccine for VL. Unlike the other cost-effectiveness papers which examine the cost-effectiveness per death averted (Boelaert et al., 1999; Meheus et al., 2010), Lee et al use disability adjusted life years (DALYs) avoided as their measure. The incremental cost-effectiveness ratio (ICER) per DALY averted is compared to the per capita gross domestic product (GDP) in Bihar of US\$288 and is designated "highly cost-effective", "costeffective" or "not cost-effective" (see Figure 13). In this analysis, the vaccine is assigned an efficacy, with $50 \%$ efficacy meaning an individual has a probability of 0.5 of becoming immune to infection following vaccination. A successfully vaccinated individual therefore will not only avoid KA but also possible PKDL.

Based on treatment with amphotericin B and an imperfect diagnostic test (rK39), Lee et al found that the vaccine was cost-effective when it cost US $\$ 100$ or less per person and had at least $25 \%$ efficacy. If the efficacy was increased to $50 \%$, and the price was less than US\$30 then vaccination could even be cost-saving compared to treatment alone. The analysis made use of a fixed ROI in simulations, as did Boelaert et al and Meheus et al. Such analyses will always underestimate the effectiveness of a vaccine, as the model does not predict any indirect protection, i.e. they assume that successfully vaccinating $50 \%$ of the population does not change the risk in the non-vaccinated (Edmunds et al., 1999). This approach also ignores the longer-term dynamic impacts of vaccination and the possibility of elimination. However, given the paucity of well validated dynamic models of VL, assuming a constant ROI is reasonable. 


\subsection{Vector}

Interventions that target vectors generally change a combination of three parameters: the biting rate on the host population, $\alpha$, vector mortality, $\mu_{V}$, and the number of vectors, $N_{V}$. Indoor residual spraying (IRS) is likely to increase mortality and hence reduce the number of vectors but might not alter the human biting rate of the remaining sandflies. The biting rate can be directly reduced by use of effective bed nets, which may be impregnated with long-lasting insecticides (LLINs). Deltamethrin-impregnated collars (DMC) on dogs provide the dogs with protection from sandfly bites via an excitorepellency effect whilst the insecticidal effect kills sandflies. At present there are no proven effective methods for reduction of sandfly populations by targeting non-adult stages. IRS, LLINs and collars only likely impact on indoor populations but not on the (outdoor) sandfly population as a whole; although this may be sufficient to reduce biting pressure to humans.

\subsubsection{On the $I S C$}

Whilst examining vector control was not the main goal of Stauch et al. (2011), it was found that a reduction of $80 \%$ of vector density would be needed in order to push $R_{0}$ below one and achieve elimination. A second, more detailed, study of vector interventions using the same model (Stauch et al., 2014) considered the impact of different types of vector control on sandfly dynamics. IRS and LLINs were both considered to kill vectors directly, increasing their mortality rate, $\mu_{V}$, which had a knock-on effect by reducing vector density $\left(N_{V} / N_{H}\right)$. Environmental vector management (EVM), linked here to plastering of houses, was assumed to destroy available breeding sites and therefore reduce the number, $N_{V}$, of vectors.

Stauch et al. (2014) found that under the first strategy (increasing mortality), elimination could be achieved if there was a reduction of $67 \%$ in sandfly density, whereas if the direct approach (through EVM) was taken, a higher reduction of $79 \%$ was needed. For the ISC in general, neither LLIN nor EVM were found to be suitable interventions on their own, as field estimates of expected density reduction were not high enough. LLIN in Bangladesh could be viable with reductions in intra-domestic sandfly density reaching 70-80\% (as measured by CDC light traps) (Joshi et al., 2009), but in India and Nepal bed nets have not had such success. IRS was found to be a feasible intervention strategy because it has been shown to reduce densities by $72 \%$ (higher than the required 67\%). The model also compared sole interventions to a combined approach and found that, for example, a $42 \%$ reduction via 
EVM (as seen in the field) would require a further $50 \%$ reduction via IRS in order to reach the critical threshold.

\subsubsection{In Africa}

ELmojtaba et al. (2010a) examine control of the vector population in their model through reducing the biting rate corresponding to interventions including bed nets or ensuring clothes cover the body. ELmojtaba et al found that vector control was by far the most effective intervention compared to various human treatment strategies (although the biting rate was reduced for both humans and reservoirs) as this control reduces not only the infectious pressure on susceptible humans but also the ROI on the sandflies from all infective individuals (including PKDL). Whilst this model does not include asymptomatics, it is interesting to note that this logic extends to them as well and it should be expected that bed nets work well at reducing the contribution of infection from all humans, regardless of whether their disease status is known/treated.

\subsubsection{In Brazil}

Dye (1996) and Ribas et al. (2013) used another similar approach to model control for humans. Both incorporated (direct) insecticidal vector control such as IRS by increasing vector mortality in their models. The results are congruent; it was found that vector control has a large impact upon prevalence in humans and was by far the best control method out of the several suggested. Even though Dye found that prevalence in the dog population was lower under treatment than under quite modest insecticide interventions, the reduction in the total bites taken caused by increasing vector mortality was sufficient that insecticide was still the best option for reducing transmission to humans where they are a spill-over host.

Ribas et al. (2013) examine the use of DMC in their model by altering the ROIs to and from dogs:

$$
\lambda_{D}=\frac{\alpha_{D}}{\theta} p_{D} \frac{I_{V}}{N_{D}} \text { and } \lambda_{V}=\frac{\alpha_{D}}{\theta} p_{V} \frac{I_{D}}{N_{D}}
$$

or under re-parameterisation

$$
\lambda_{D}=\alpha_{D}(1-B) p_{D} \frac{I_{V}}{N_{D}} \text { and } \lambda_{V}=\alpha_{D}(1-B) p_{V} \frac{I_{D}}{N_{D}}
$$

where $\frac{1}{\theta}=(1-B)$ and $0 \leq B \leq 1$. 
Here $B$ can be though of as the proportion reduction in biting. Using this model structure, Ribas et al found that collaring was reasonably effective at prevention infection and could dramatically reduce prevalence in humans if the protection offered by the collars was high.

Figure 14: Model of collaring by Reithinger et al. (2004). The subscript $C D$ represents those dogs which have been collared, which occurs at $\rho_{C}=80 \%$ of the dog population per year (in a single pulse). Collars loose effectiveness or are removed/lost at a continuous rate of $\xi_{C}=0.001$ per day. Collared dogs have $90 \%$ protection from sandfly bites.

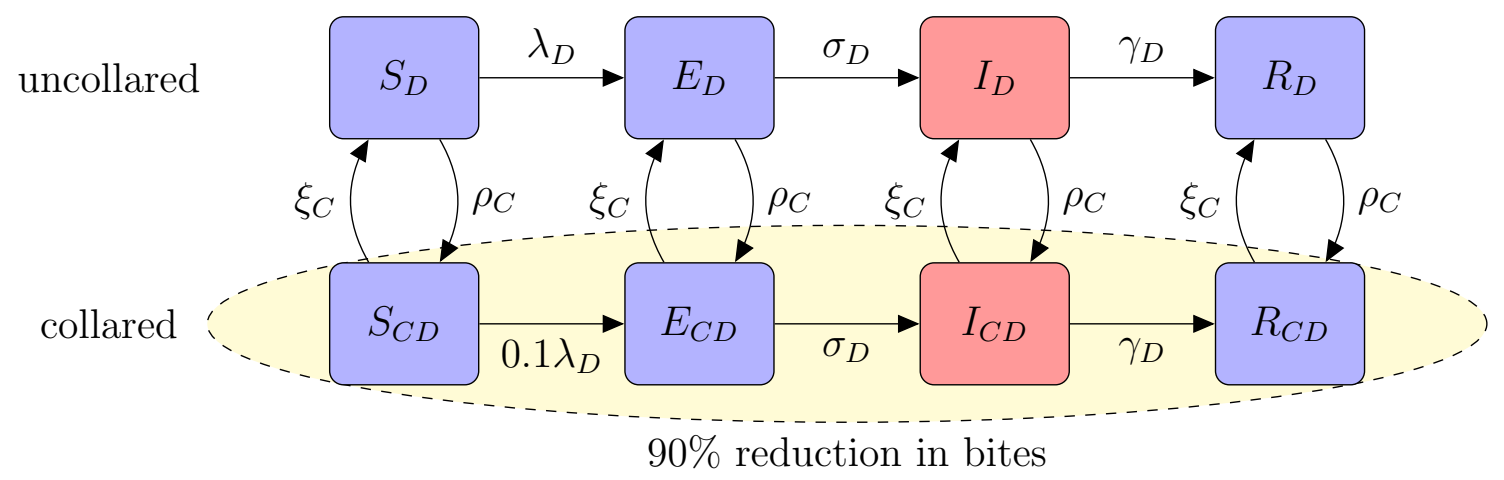

Reithinger et al. (2004) took an alternative approach by adding an addition compartment to their model. Here pulse collaring of $80 \%$ of dogs was simulated either 1 or 2 times per year with DMC losing effectiveness continuously over 8 months. The results were found to be dependent upon the demography of the dog population with a lower average life expectancy making collars a more effective strategy. Simulated DMC effects did not vary greatly with change in endemicity. In contrast, baseline prevalence levels impacted quite substantially upon culling success.

These DMC models examined a reduction in biting for dogs with collars but did not consider the possible effects of either an associated increase in mortality of sandflies from feeding attempts on collared dogs, or displaced bites (and subsequently higher biting) on uncollared dogs. These two additional effects might be expected to decrease or increase the total prevalence respectively. Insecticidal effects of collars would not stop transmission from infected flies to susceptible dogs but would prevent dog to fly transmission as sandflies would either not feed or die before passing through their EIP. 


\subsection{Dogs}

\subsubsection{Diagnostics}

Courtenay et al. (2002b) examined the infectious states of a naturally exposed cohort of dogs by xenodiagnoses in order to appropriately model the dog population by partitioning into never/ever-infectious categories (used by Hasibeder et al., 1992). They used the sensitivity/specificity of the different diagnostic tools to simulate which dogs would be identified as seropositive during screening. The authors found that insensitivity of the diagnostic test, as well as delays between diagnosis and culling, could explain why a culling intervention might fail. In a follow-on study, Courtenay et al. (2014) used qPCR to quantify heterogeneities in L. infantum burdens in canine tissues to relate to their infectiousness to sandflies, and demonstrated that qPCR could identify the majority of infectious dogs and potentially distinguish highly infectious from non-infectious animals. In their model they demonstrated that a small proportion of $\operatorname{dog}$ s were responsible for the majority of transmission events.

Costa et al. (2013a) use the sensitivity and specificity of diagnostics in their model which includes infectious symptomatic and asymptomatic dogs (with asymptomatic dogs assumed to be 3 fold less infective to sandflies than those with symptoms), as described previously and visualised in Figure 10. Under the model the sensitivity and specificity of the diagnostic were both assumed to be within a range of $80-100 \%$ and were assumed to be the same for symptomatic and asymptomatic dogs (which is unlikely to be true). The model results indicate that the presence of asymptomatic dogs impacted the control strategies and time scales negatively; the authors concluded that models without asymptomatics might over-estimate the effectiveness of interventions reliant on diagnostic tools such as culling. In higher prevalence areas the control of the asymptomatic dog population was necessary to reduce transmission below the threshold level. In this case high sensitivity tests would be needed to find the asymptomatic reservoir, whilst high specificity tests reduce the numbers of dogs needed to be culled and critically, reduce the unnecessary culling of false positives. This indicates that optimal strategy is likely to depend upon regional prevalence. As screening is costly it is likely that this would only be cost-effective in higher prevalence areas.

\subsubsection{Treatment}

Unlike VL in humans, treatment of disease in dogs is not part of national control programmes: nevertheless it is possible to treat dogs for infection 
with drugs. Only two articles pertaining to dogs included the effect of treatment of infected dogs in their models (Dye, 1996; Ribas et al., 2013). In both articles, the same approach was taken, whereby a constant treatment rate was assumed, presumably corresponding to ad hoc detection of infected dogs via their symptoms. However Ribas et al assume that dogs will become susceptible to disease once more post-treatment rather than retaining immunity from re-infection as assumed by Dye. This difference has huge implications for modelling results and explains the stark discrepancy in conclusions drawn by these authors; Ribas et al found that treatment of infected dogs had virtually no impact on overall prevalence of infection (in humans) whereas Dye found it to be moderately beneficial at reducing prevalence. In reality, dogs may relapse to a diseased state, not through re-infection, but rather through recrudescence of parasites post-treatment as treatment is rarely curative.

\subsubsection{Dog culling}

Unlike the strategies to control VL in humans, there are alternative options for intervention in animal populations. The first of these interventions is to cull infected dogs in order to reduce the ROI towards the sandfly; this intervention has been posed as a viable strategy in Brazil and has had seemingly beneficial effects in China (when employed concurrently with insecticide) (Zhi-Biao, 1989). Some authors (Dye, 1996; Palatnik-de Sousa et al., 2004; Costa et al., 2013a) implement culling in models in a similar way to which treatment is modelled by Ribas et al. (2013), that is, a diagnosis of infection is made either via symptoms or ongoing screening and culling will subsequently occur at some constant rate throughout the year with the removed dogs being replaced with an influx of susceptible dogs (or puppies). In contrast, other authors (Courtenay et al., 2002b; Reithinger et al., 2004) opt to model a pulse culling campaign where dogs are annually removed after mass screening (50\% screening in Reithinger and 100\% in Courtenay). Courtenay et al assume that removed dogs are "rapidly" replaced by susceptible ones, while Reithinger et al use a dog recruitment method where the population returns to equilibrium over time at a rate described by:

$$
\bar{\mu}_{V} N_{V}+\beta\left(1-\frac{N_{V}}{K}\right) N_{V}
$$

where $\bar{\mu}_{V}$ is the weighted average mortality rate from both natural death and infection, $\beta$ is the density dependent growth rate of the population and $K$ is the carrying capacity of the population at equilibrium. 
The second form of replacement is more realistic as an area could not replace all their dogs instantly. As culled dogs will be replaced with susceptible ones, unsurprisingly most authors conclude that culling is not likely to be the best option available. Dye (1996) found it to be the worse option amongst all posed strategies by using a maximum cull rate of infectious dogs of around $4 \%$ per month in his analysis. Costa et al, used a $4 \%$ screening of symptomatic dogs per month (which would result in an even smaller proportion being culled) with a 4-month delay before culling. They found that whilst this was sufficient to reduce transmission to less than $1 \%$ in low transmission settings, that high-endemicity regions would need to cull a minimum of $30 \%$ of asymptomatically (but infectious) dogs to achieve the same results. Costa et al also emphasised how, if screening the whole dog population, $79 \%$ of those culled would have been removed unnecessarily using a diagnostic test with $80 \%$ specificity.

Palatnik-de-Souza et al and Ribas et al examine much higher rates of culling assuming homogeneity in infectiousness with the former determining that $7 \%$ of infectious dogs must be removed per day to reduce $R_{0}$ below the critical threshold of one, and the latter concluding that below $18 \%$ culling per day there would be virtually no impact on disease transmission to humans.

One group, Reithinger et al. (2004), found culling to be moderately effective at reducing prevalence of infection in dogs. In particular, increasing the percentage of infected dogs culled and/or the frequency of screening campaigns (from one to two pulses per year), made a significant reduction to the prevalence. Culling had higher relative effectiveness in low transmission settings compared to high transmission areas.

In summary of existing culling models, culling can be made more effective by reducing time between diagnosis and culling, and by improving the sensitivity so that a higher percentage of infectious dogs are identified. There needs to be a (perhaps unrealistically) high screening coverage and rate to achieve substantial reduction in disease burden. Specificity of diagnostics must be increased if all dogs are to be screened in order to reduce incorrect diagnosis and culling of non-infectious dogs which in turn should increase dog owner compliance (Costa et al., 2013a; Courtenay et al., 2014). In particular it would useful to have a tool to differentiate between infected and infectious dogs to minimise the level of culling needed and target this control effectively (Courtenay et al., 2002b). Culling of healthy uninfectious dogs should be avoided from an ethical perspective. 


\subsubsection{Dog vaccination}

Often when vaccination is considered in models it is assumed to be prophylactic with individuals gaining immunity (or partial immunity) with successful inoculation. Such a (fully) prophylactic vaccine model is used by Dye (1996) to examine the potential effect of immunisation of a dog population; he found that at low levels of intervention, vaccination would be one of the best strategies, although insecticides may more easily eliminate disease at higher levels of intervention. Under Dye's parameterisation, around 90\% of the susceptible dog population would have to be successfully vaccinated in order to achieve elimination.

Aside from prophylactic vaccine, other types of vaccine which have been developed for other diseases could potentially provide an alternative intervention for VL. One which has been modelled in a dog population is a transmission-blocking vaccine, in which a vaccinated dog may still acquire infection, but upon becoming infected they will have a reduced infectivity towards sandflies through reduced development of the parasite within the vector (Ribas et al., 2013).

In the model by Ribas et al Ribas et al. (2013), the ROI towards the vector is amended from:

$$
\lambda_{V}=\alpha_{D} p_{V} \frac{I_{D}}{N_{D}}
$$

to includ this reduced infectivity:

$$
\lambda_{V}=\alpha_{D} \frac{p_{V}}{\nu} \frac{I_{D}}{N_{D}}
$$

or under re-parameterisation:

$$
\lambda_{V}=\alpha_{D}(1-T) p_{V} \frac{I_{D}}{N_{D}}
$$

where $\frac{1}{\nu}=(1-T)$ and $0 \leq T \leq 1$.

Here, $T$ might represent the vaccine efficacy, i.e. $T=1$ is $100 \%$ effective at blocking transmission and $T=0$ is $0 \%$ effective given all the dog population is vaccinated. Alternatively $T$ could be the proportion of successfully vaccinated dogs in the population. In the first case the infectivity parameter, $p_{V}$ is essentially reduced by a factor of $(1-T)$, whereas in the latter, just a proportion $(1-T)$ of dogs remain unvaccinated and since vaccination does not prevent dogs becoming infected, the same proportion, $(1-T)$ are fully 
infective to dogs whilst the rest are non-infective. Either of these results in the same mathematical formulation of the ROI.

Under this formulation, the transmission-blocking vaccine was simulated and found to be marginally better at reducing prevalence in humans (considered to be a spill-over host) than treatment of dogs, however as the intervention impacts only on infectivity of some blood-meals and does not directly affect sandfly feeding rates or mortality, the intervention did not have a pronounced effect upon human prevalence.

In the case of VL, transmission-blocking vaccines may also prevent disease in dogs (but not infection) as both symptoms and infectiousness are correlated with parasite burden. Consequently there may be less of a distinction between prophylactic and transmission-blocking vaccines for ZVL. Future canine vaccines may also have a curative effect if given to a diseased dog; this effect has not been explicitly modelled.

\subsection{Other animal hosts}

ELmojtaba et al. (2010a) found that the presence of other animal populations which are able to acquire and transmit VL would increase the prevalence in humans, however at present no other animals have been shown to be reservoirs (Quinnell and Courtenay, 2009). The presence of additional host populations and their impact on the primary population is non-trivial; in large enough numbers the secondary host population removes biting pressure away from the primary hosts (humans) and consequently this may in fact reduce the ROI upon primary hosts. Conversely the additional population(s) may drive infection in the primary hosts, or, in this case, stifle the effects of control upon just the primary population through treatment of infective VL humans. Under the parameterisation of ELmojtaba et al. (2010a) the removal of this secondary population was beneficial to humans although the reduction in $R_{0}^{N G M}$ was relatively paltry (3.4 down to 2.3) and indicates that whilst infection is boosted by the animal reservoir, it is not sustained by it.

\section{Conclusions}

Despite model predictions based on well defined (average) parameter estimates and scenarios, the operational realities on the ground are somewhat different adding a variable complexity that are difficult to capture. VL disease on the ISC has been targeted for elimination as a public health problem, but 
there are still many facets of this infection which are not well understood. In particular the progression of disease through different stages, including asymptomatic infection and PKDL, is unclear, especially in relation to diagnosis and infectivity. Recent efforts to control VL on the ISC seem to have had success, prompting the original goal of elimination as a public health problem to be brought forwards from 2020 to 2017 (WHO, 2013; Boni et al., 2014). However, there are also indications that caution as well as optimism should be exercised; modelling and transmission studies have indicated a credible possibility that asymptomatically infected individuals might hinder elimination efforts or obscure the true number of those with Leishmania infection.

Treatment of people presenting with $\mathrm{KA}$ is central to the current interventions. Models disagree on whether this intervention alone is adequate to achieve control and sufficiently reduce incidence. This disagreement hinges on the relative proportion of transmission to sandflies due to symptomatic people who are then treated. If KA patients are responsible for the majority of transmission, then treatment will have a big impact on onward transmission. The evidence for this situation is indirect and largely reliant on the spatio-temporal clustering of KA cases and the long period cycles seen at regional levels; both of these are more easily explained if KA cases are responsible for transmission. However, if the majority of transmission is due to asymptomatic infections, then treating KA patients promptly will have little impact on transmission. There is less evidence for this situation, but it is not unlikely if there are large numbers of asymptomatic infections that transmit to sandflies at a low rate. This situation highlights the need for more accurate data on the natural history and relative infectiousness of different infection states.

Looking forwards, model predictions of vector control consistently result in effective control of disease, not just on the ISC but in regions affected by ZVL too. IRS seems to have the maximal benefit amongst strategies including bed net use and other EVM. However it is emphasised that these modelling results do depend critically on the infectivity of different disease states, the effectiveness of IRS and the vector response to EVM. Again, there is a paucity of data to support parameterisation of these processes in any model. With control of sandfly populations appearing pivotal in the elimination campaign, it will be especially important to improve both our understanding of sandfly biology and how transmission may change through the course of a year. Future modelling of VL in humans could utilise the 
basic frameworks set out by those who have previously modelled seasonality of sandflies in ZVL as well as the wider literature on seasonal dependence of other disease vectors.

Modelling of VL in dogs has shown that the sensitivity and specificity of diagnostics are likely to have a direct impact on the success of culling strategies for dogs, and hints at the wider importance of accurate diagnostic tools in reporting and control human disease. Caution must be taken with modelled intervention comparisons because, in reality, some interventions may be far easier to employ (to the extent needed) than others. This is highlighted in the canine VL papers which have mixed suggestions for the best intervention.

The current intervention programmes focus on eliminating VL disease as a public health problem rather than considering achieving a break in transmission. Elimination as a pubic health problem is technically described as control in that continued efforts are required to maintain the lower incidence. As the numbers of cases on the ISC falls towards the target of 1 per 10,000, clinical awareness is likely to fall. Additionally, if there is population (herd) immunity that currently reduces transmission (because a proportion of the population are immune), then this too may wane over time. As a consequence it is possible that there will be an increasingly susceptible population with a less alert medical and public health system and on-going transmission, all within a matter of years. This is the situation in which transmission can resurge and potentially large epidemics occur. Therefore it is important to understand transmission rather than focus solely on diseased individuals to ensure recrudescence does not occur.

There are several key knowledge and data gaps which have been identified within this chapter. There is a need to better understand the biology of disease including disease progression (by determining markers to differentiate self-clearing asymptomatic infection from those that progress to disease), infectivity of all infected individuals, and prospects for human vaccine development. However there is also a need to better inform models with data; models need to consider time-dependent transients, which emphasises the importance of longitudinal data. The important dimensions of heterogeneity, including individual-level differences and larger-scale spatial aspects, need to be incorporated in models if they are to capture key aspects of transmission dynamics.

Unsurprisingly, the number of models of visceral leishmaniasis is extremely low, especially when compared to the vast modelling literature for 
other vector-borne disease such as malaria and dengue. However, the existing models do address a range of important questions such as the level of underreporting of VL disease and relative impact of different interventions. Future models must be fitted to high quality contemporary data sets in order to ensure the highest level predictive value of the results and hopefully, through good communication between modellers and the larger VL community, the key outstanding questions may be addressed. 
Table 5: Summary of mathematical modelling articles of VL extended from Rock et al. (2015a).

Anthroponotic

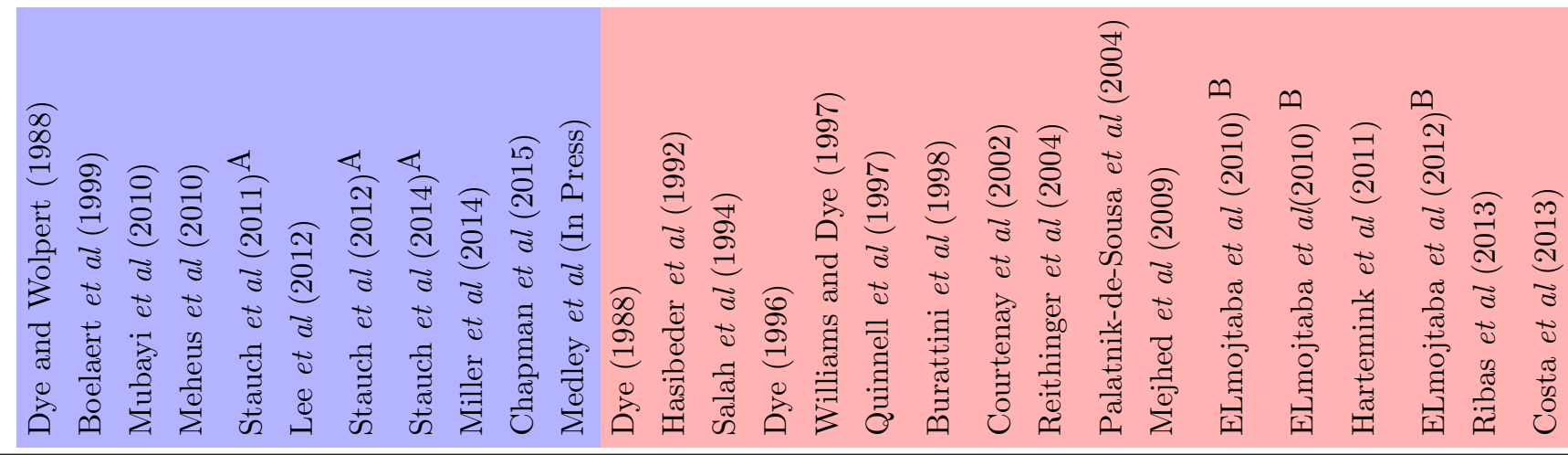

8

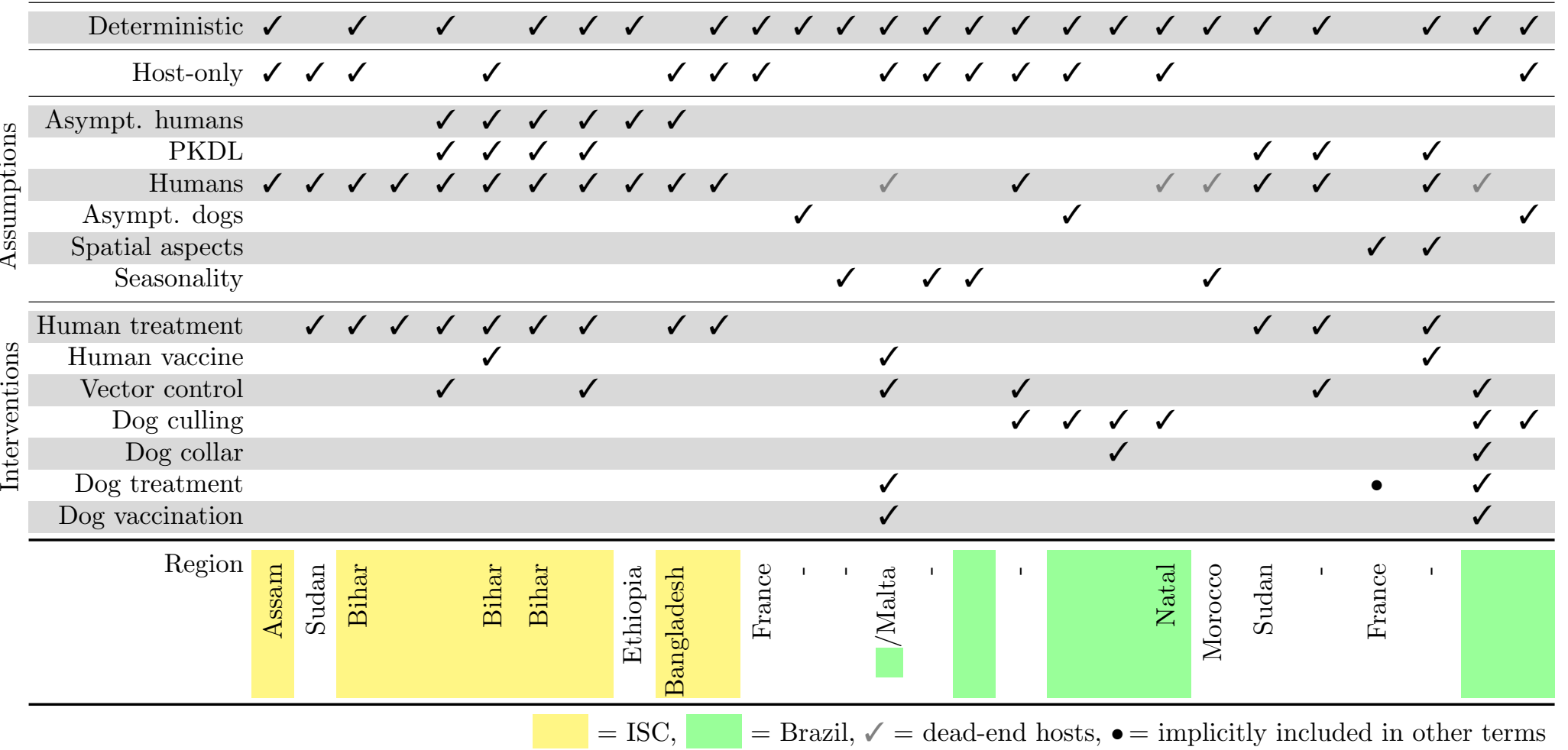




\section{Acknowledgements}

The authors would like to thank Dr Lloyd A. C. Chapman for his comments on the manuscript and Dr T. Deirdre Hollingsworth for her involvement with VL research under the Diagnostics Modelling Consortium and NTD Modelling Consortium. KSR was funded for this work under the Diagnostics Modelling Consortium funded by the Bill \& Melinda Gates Foundation, and OC and RJQ acknowledge the long standing support for field studies by the Wellcome Trust. KSR, GFM and OC gratefully acknowledge funding by the Bill and Melinda Gate Foundation in partnership with the Task Force for Global Health. The views, opinions, assumptions or any other information set out in this article are solely those of the authors.

\section{References}

Adams, E. R., Versteeg, I., Leeflang, M. M. G., 2013. Systematic review into diagnostics for post-kala-azar dermal leishmaniasis (PKDL). Journal of Tropical Medicine 2013, Article ID 150746.

Ahluwalia, I. B., Bern, C., Costa, C. H. N., Akter, T., Chowdhury, R., Ali, M., Alam, D., Kenah, E., Amann, J., Islam, M., Wagatsuma, Y., Haque, R., Breiman, R. F., Maguire, J. H., 2003. Visceral leishmaniasis: Consequences of a neglected disease in a Bangladeshi community. American Journal of Tropical Medicine and Hygiene 69, 624-628.

Alam, M. S., Ghosh, D., Khan, G. M., Islam, M. F., Mondal, D., Itoh, M., Islam, N., Haque, R., Jun. 2011. Survey of domestic cattle for antiLeishmania antibodies and Leishmania DNA in a visceral leishmaniasis endemic area of Bangladesh. BMC Veterinary Research 7 (1), 27.

Alexander, B., de Carvalho, R. L., McCallum, H., Pereira, M. H., 2002. Role of the domestic chicken (Gallus gallus) in the epidemiology of urban visceral leishmaniasis in Brazil. Emerging Infectious Diseases 8, 1-6.

Alexander, B., Maroli, M., 2003. Control of phlebotomine sandflies. Medical and Veterinary Entomology 17, 1-18.

Ali, M., Ashford, R. W., 1994. Visceral leishmaniasis in Ethiopia. IV. Prevalence, incidence and relation of infection to disease in an endemic area. Annals of Tropical Medicine and Parasitology 88, 289-293. 
Alten, B., Caglar, S. S., Kaynas, S., Simsek, F. M., 2003. Evaluation of protective efficacy of K-OTAB impregnated bednets for cutaneous leishmaniasis control in Southeast Anatolia-Turkey. Journal of Vector Ecology $28,53-64$.

Alvar, J., Aparicio, P., Aseffa, A., den Boer, M., Canavate, C., Dedet, J.P., Gradoni, L., Ter Horst, R., Lopez-Velez, R., Moreno, J., Apr. 2008. The Relationship between Leishmaniasis and AIDS: the Second 10 Years. Clinical Microbiology Reviews 21 (2), 334-359.

Alvar, J., Vélez, I. D., Bern, C., Herrero, M., Desjeux, P., Cano, J., Jannin, J. G., Boer, M. d., the WHO Leishmaniasis Control Team, May 2012. Leishmaniasis worldwide and global estimates of its incidence. PLoS One 7 (5), e35671.

Anderson, R. M., May, R. M., 1992. Infectious Diseases of Humans: Dynamics and Control. Oxford University Press.

Andrade, M. S., Courtenay, O., Brito, M. E. F., Carvalho, F. G., Carvalho, A. W. S., Soares, F. C. S., Carvalho, S. M. S., Costa, P. L., Zampieri, R. A., Floeter-Winter, L. M., Shaw, J. J., Brandao-Filho, S. P., 2015. Infectiousness of sylvatic and synanthropic small rodents implicates a multi-host reservoir of Leishmania (Viannia) braziliensis). PLoS Neglected Tropical Diseases 9 (10), e4137.

Anstead, G. M., Chandrasekar, B., Zhao, W., Yang, J., Perez, L. E., Melby, P. C., Aug. 2001. Malnutrition alters the innate immune response and increases early visceralization following Leishmania donovani infection. Infection and Immunity 69 (8), 4709-4718.

Antoniou, M., Messaritakis, I., Christodoulou, V., Ascoksilaki, I., Kanavakis, N., Sutton, A. J., Carson, C., Courtenay, O., Jun. 2009. Increasing Incidence of Zoonotic Visceral Leishmaniasis on Crete, Greece. Emerging Infectious Diseases 15 (6), 932-934.

Aoun, K., Chouihi, E., Amri, F., Alaya, N. B., Raies, A., Mary, C., Bouratbine, A., Dec. 2009. Contribution of Quantitative Real-Time Polymerase Chain Reaction to Follow-Up of Visceral Leishmaniasis Patients Treated with Meglumine Antimoniate. American Journal of Tropical Medicine and Hygiene 81 (6), 1004-1006. 
Bacaër, N., Jan. 2007. Approximation of the basic reproduction number $R_{0}$ for vector-borne diseases with a periodic vector population. Bulletin of Mathematical Biology 69 (3), 1067-1091.

Bacaër, N., Guernaoui, S., Jul. 2006. The epidemic threshold of vector-borne diseases with seasonality. Journal of Mathematical Biology 53 (3), 421436.

Badaro, R., Jones, T. C., Carvalho, E. M., Sampaio, D., Reed, S. G., Barral, A., Teixeira, R., Johnson, W. D., 1986a. New perspectives on a subclinical form of visceral leishmaniasis. Journal of Infectious Diseases 154, 10031011.

Badaro, R., Jones, T. C., Teixeira, H. R. R., Johnson, W. D., 1986b. A prospective study of visceral leishmaniasis in an endemic area of Brazil. Journal of Infectious Diseases 154 (4), 639-649.

Baneth, G., Koutinas, A. F., Solano-Gallego, L., Bourdeau, P., Ferrer, L., Jul. 2008. Canine leishmaniosis - new concepts and insights on an expanding zoonosis: part one. Trends in Parasitology 24 (7), 324-330.

Barnett, P. G., Singh, S. P., Bern, C., Hightower, A. W., Sundar, S., 2005. Virgin soil: The spread of visceral leishmaniasis into Uttar Pradesh, India. American Journal of Tropical Medicine and Hygiene 73, 720-725.

Barral, A., Badaro, R., Barral-Netto, M., Grimaldi, G., Momem, H., Carvalho, E. M., 1986. Isolation of Leishmania mexicana amazonensis from the bone marrow in a case of American visceral leishmaniasis. American Journal of Tropical Medicine and Hygiene 35, 732-734.

Bejon, P., Williams, T. N., Nyundo, C., Hay, S. I., Benz, D., Gething, P. W., Otiende, M., Peshu, J., Bashraheil, M., Greenhouse, B., Bousema, T., Bauni, E., Marsh, K., Smith, D. L., Borrmann, S., 2014. A micro-epidemiological analysis of febrile malaria in coastal Kenya showing hotspots within hotspots. eLife 3, e02130.

Benzerroung, E. H., Benhabylles, N., Izri, M. A., Belahcene, E. K., 1992. Les pulverisations intra- et peri-domiciliaires de DDT dans la lutte contre la leishmaniose cutanee zoonotique en Algerie. Annales de la Societe Belge de Medecine Tropicale 72, 5-12. 
Bern, C., Amann, J., Haque, R., Chowdhury, R., Ali, M. Y., Kurkijian, K. M., Vaz, L., Wagatsumam, Y., Breiman, R. F., Secor, W. E., Maguire, J., 2006. Loss of Leishmanin skin test antigen sensitivity and potency in a longitudinal study of visceral leishmaniasis in Bangladesh. American Journal of Tropical Medicine and Hygiene 75, 744-748.

Bern, C., Chowdhury, R., 2006. The epidemiology of visceral leishmaniasis in Bangladesh: prospects for improved control. Indian Journal of Medical Research A 123, 275-288.

Bern, C., Courtenay, O., Alvar, J., Feb. 2010. Of cattle, sand flies and men: A systematic review of risk factor analyses for South Asian visceral leishmaniasis and implications for elimination. PLoS Neglected Tropical Diseases 4 (2), e599.

Bern, C., Haque, R., Chowdhury, R., Ali, M., Kurkijian, K. M., Vaz, L., Amann, J., Wahed, M. A., Wagatsumam, Y., Breiman, R. F., Williamson, J., Secor, W. E., Maguire, J. H., 2007. The epidemiology of visceral leishmaniasis and asymptomatic leishmanial infection in a highly endemic Bangladeshi village. American Journal of Tropical Medicine and Hygiene 76, 909-914.

Bern, C., Hightower, A. W., Chowdhury, R., Ali, M., Amann, J., 2005. Risk factors for kala-azar in Bangladesh. Emerging Infectious Diseases 11, 655662.

Bhattarai, N. R., Van der Auwera, G., Rijal, S., Picado, A., Speybroeck, N., Khanal, B., De Doncker, S., Das, M. L., Ostyn, B., Davies, C. R., Coosemans, M., Berkvens, D., Boelaert, M., Dujardin, J.-C., Feb. 2010. Domestic Animals and Epidemiology of Visceral Leishmaniasis, Nepal. Emerging Infectious Diseases 16 (2), 231-237.

Biglino, A., Bolla, C., Concialdi, E., Trisciuoglio, A., Romano, A., Ferroglio, E., Jan. 2010. Asymptomatic Leishmania infantum infection in an area of northwestern Italy (Piedmont Region) where such infections are traditionally nonendemic. Journal of Clinical Microbiology 48 (1), 131-136.

Boelaert, M., Lynen, L., Desjeux, P., Van der Stuyft, P., Aug. 1999. Costeffectiveness of competing diagnostic-therapeutic strategies for visceral leishmaniasis. Bulletin of the World Health Organization 77 (8), 667-674. 
Boelaert, M., Meheus, F., Sanchez, A., Singh, S. P., Vanlerberghe, V., Picado, A., Meessen, B., Sundar, S., Jun. 2009. The poorest of the poor: a poverty appraisal of households affected by visceral leishmaniasis in Bihar, India. Tropical Medicine and International Health 14 (6), 639-644.

Boni, M., Pratlong, F., El Hadi Osman, M., Bucheton, B., El-Safi, S., Feugier, E., Musa, M. K., Davoust, B., Dessein, A., Dedet, J.-P., 2014. Report of the 67th session. WHO Regional Committee for South-East Asia, $1-157$.

Borja-Cabrera, G. P., Correia Pontes, N. N., da Silva, V. O., Paraguai de Souza, E., Santos, W. R., Gomes, E. M., Luz, K. G., Palatnik, M., Palatnik-de Sousa, C. B., 2002. Long lasting protection against canine kala-azar using the FML-QuilA saponin vaccine in an endemic area of Brazil (Sao Goncalo do Amarante, RN). Vaccine 20, 3277-3284.

Borja-Cabrera, G. P., Cruz Mendes, A., Paraguai de Souza, E., Hashimoto Okada, L. Y., de A Trivellato, F. A., Kawasaki, J. K. A., Costa, A. C., Reis, A. B., Genaro, O., Batista, L. M. M., Palatnik, M., Palatnik-de Sousa, C. B., Jun. 2004. Effective immunotherapy against canine visceral leishmaniasis with the FML-vaccine. Vaccine 22 (17-18), 2234-2243.

Bray, D. P., Carter, V., Alves, G. B., Brazil, R. P., Bandi, K. K., Hamilton, J. G. C., Mar. 2014. Synthetic Sex Pheromone in a Long-Lasting Lure Attracts the Visceral Leishmaniasis Vector, Lutzomyia longipalpis, for up to 12 Weeks in Brazil. PLoS Neglected Tropical Diseases 8 (3), e2723.

Burza, S., Sinha, P. K., Mahajan, R., Lima, M. A., Mitra, G., Verma, N., Balsegaram, M., Das, P., Jan. 2014. Risk Factors for Visceral Leishmaniasis Relapse in Immunocompetent Patients following Treatment with $20 \mathrm{mg} / \mathrm{kg}$ Liposomal Amphotericin B (Ambisome) in Bihar, India. PLoS Neglected Tropical Diseases 8 (1), e2536.

Carneiro, M., Moreno, E. C., Goncalves, A. V., Lambertucci, K. R., Antunes, C. M. F., 2011. Visceral leishmaniasis: challenges in identifying subclinical Leishmania infection . Drug development research 72 (6), 442-450.

Casanova, C., Natal, D., Santos, F. A. M., 2009. Survival, Population Size, and Gonotrophic Cycle Duration of Nyssomyia neivai (Diptera: Psychodidae) at an Endemic Area of American Cutaneous Leishmaniasis in Southeastern Brazil. Journal of Medical Entomology 46, 42-50. 
Cerf, B. J., Jones, T. C., Badaro, R., Sampaio, D., Teixeira, R., Johnson, W. D., 1987. Malnutrition as a risk factor for severe visceral leishmaniasis. Journal of Infectious Diseases 156 (6), 1030-1033.

Chapman, L. A., Dyson, L., Hollingsworth, T. D., Courtenay, O., Bern, C., Medley, G. F., 2015. Quantification of the natural history of visceral leishmaniasis and consequences for control. Parasites \& Vectors.

Chowdhury, R., Huda, M. M., Kumar, V., Das, P., Joshi, A. B., Banjara, M. R., Akhter, S., Kroeger, A., Krishnakumari, B., Petzold, M., Mondal, D., Das, M. L., Jan. 2011. The Indian and Nepalese programmes of indoor residual spraying for the elimination of visceral leishmaniasis: performance and effectiveness. Annals of Tropical Medicine and Parasitology 105 (1), $31-35$.

Chunge, C. N., Owate, J., Pamba, H. O., Donno, L., Mar. 1990. Treatment of visceral leishmaniasis in Kenya by aminosidine alone or combined with sodium stibogluconate. Transactions of the Royal Society of Tropical Medicine and Hygiene 84 (2), 221-225.

Coleman, M., Foster, G. M., Deb, R., Singh, R. P., Ismail, H. M., Shivam, P., Ghosh, A. K., Dunkley, S., Kumar, V., Coleman, M., Hemingway, J., Paine, M. J. I., Das, P., Jul. 2015. DDT-based indoor residual spraying suboptimal for visceral leishmaniasis elimination in India. PNAS 112 (28), 8573-8578.

Collin, S. M., Coleman, P. G., Ritmeijer, K., Davidson, R. N., Mar. 2006. Unseen Kala-azar deaths in south Sudan (1999-2002). Tropical Medicine and International Health 11 (4), 509-512.

Costa, C. H. N., 2011. How effective is dog culling in controlling zoonotic visceral leishmaniasis? A critical evaluation of the science, politics and ethics behind this public health policy. Revista da Sociedade Brasileira de Medicina Tropical 44 (2), 232-242.

Costa, C. H. N., Gomes, R. B. B., Silva, M. R. B., Garcez, L. M., Ramos, P. K. S., Santos, R. S., Shaw, J. J., David, J. R., Maguire, J. H., 2000. Competence of the human host as a reservoir for Leishmania chagasi. Journal of Infectious Diseases 182, 997-1000. 
Costa, D. N. C. C., Codeço, C. T., Silva, M. A., Werneck, G. L., Aug. 2013a. Culling dogs in scenarios of imperfect control: realistic impact on the prevalence of canine visceral leishmaniasis. PLoS Neglected Tropical Diseases 7 (8), e2355.

Costa, P. L., Dantas-Torres, F., da Silva, F. J., Guimarães, V. C. F. V., Gaudêncio, K., Brandao-Filho, S. P., May 2013b. Ecology of Lutzomyia longipalpis in an area of visceral leishmaniasis transmission in north-eastern Brazil. Acta Tropica 126 (2), 99-102.

Courtenay, O., Carson, C., Calvo-Bado, L., Garcez, L. M., Quinnell, R. J., Jan. 2014. Heterogeneities in Leishmania infantum infection: Using skin parasite burdens to identify highly infectious dogs. PLoS Neglected Tropical Diseases 8 (1), e2583.

Courtenay, O., Gillingwater, K., Gomes, P. A., Garcez, L. M., Davies, C. R., 2007. Deltamethrin-impregnated bednets reduce human landing rates of sandfly vector Lutzomyia longipalpis in Amazon households. Medical and Veterinary Entomology 21 (2), 168-176.

Courtenay, O., Quinnell, R. J., Garcez, L. M., Dye, C., 2002a. Low infectiousness of a wildlife host of Leishmania infantum: the crab-eating fox is not important for transmission. Parasitology 125, 407-414.

Courtenay, O., Quinnell, R. J., Garcez, L. M., Shaw, J. J., Dye, C., 2002b. Infectiousness in a cohort of Brazilian dogs: Why culling fails to control visceral leishmaniasis in areas of high transmission. Journal of Infectious Diseases 186, 1314-1320.

Croft, S. L., Sundar, S., Fairlamb, A. H., Jan. 2006. Drug Resistance in Leishmaniasis. Clinical Microbiology Reviews 19 (1), 111-126.

Cruz-Pacheco, G., Esteva, L., Vargas, C., Jun. 2012. Control measures for Chagas disease. Mathematical Biosciences 237 (1-2), 49-60.

Cunningham, J., Hasker, E., Das, P., El-Safi, S., Goto, H., Mondal, D., Mbuchi, M., Mukhtar, M., Rabello, A., Rijal, S., Sundar, S., Wasunna, M., Adams, E., Menten, J., Peeling, R., Boelaert, M., for the WHO/TDR Visceral Leishmaniasis Laboratory Network, Oct. 2012. A global comparative evaluation of commercial immunochromatographic rapid diagnostic 
tests for visceral leishmaniasis. Clinical Infectious Diseases 55 (10), 13121319.

da Saúde Secretaria de Vigilância em Saúde Departamento de Vigilância Epidemiológica, 2006. Manual de Vigilancia e Controle da Leishmaniose Visceral. Tech. rep.

Das, M., Saudagar, P., Sundar, S., Dubey, V. K., Aug. 2013. Miltefosineunresponsive Leishmania donovani has a greater ability than miltefosineresponsive $L$. donovanito resist reactive oxygen species. FEBS Journal 280 (19), 4807-4815.

Davies, C. R., Llanos-Cuentas, E. A., Campos, P., Monge, J., Leon, E., Canales, J., 2000. Spraying houses in the Peruvian Andes with lambdacyhalothrin protects residents against cutaneous leishmaniasis. Transactions of the Royal Society of Tropical Medicine and Hygiene 94, 631-636.

Davies, C. R., Mazloumi Gavgani, A. S., 1999. Age, acquired immunity and the risk of visceral leishmaniasis: a prospective study in Iran. Parasitology $119,247-257$.

de Colmenares, M., Portus, M., Botet, J., Dobano, C., Gallego, M., Wolff, M., Segui, G., 1995. Identification of blood meals of Phlebotomus perniciosus (Diptera: Psychodidae) in Spain by a competitive enzyme-linked immunosorbent assay biotin/avidin method. Journal of Medical Entomology 32 (3), 229-233.

de Gouvea Viana, L., de Assiss, T. S., da Silva, A. R., de Souza, G. F., Caligiorne, R., da Silva, A. C., Peruhype-Magalhaes, V., Marciano, A. P., Martins-Filho, O. A., Rabello, A., 2008. Combined diagnostic methods identify a remarkable proportion of asymptomatic Leishmania (Leishmania) chagasi carriers who present modulated cytokine profiles. Transactions of the Royal Society of Tropical Medicine and Hygiene 102, 548-555.

de Melo, E. C., Fortaleza, C. M. C. B., 2013. Challenges in the Therapy of Visceral Leishmaniasis in Brazil: A Public Health Perspective. Journal of Tropical Medicine 2013 (1), Article ID 319234.

de Ruiter, C. M., van der Veer, C., Leeflang, M. M. G., Deborggraeve, S., Lucas, C., Adams, E. R., Aug. 2014. Molecular tools for diagnosis of vis- 
ceral leishmaniasis: Systematic review and meta-analysis of diagnostic test accuracy. Journal of Clinical Microbiology 52 (9), 3147-3155.

de Souza, V. M. M., da Silva Juliao, F., Neves, R. C. S., Magalhaes, P. B., Bisinotto, T. V., de Souza Lima, A., de Oliveira, S. S., Junior, E. D. M., 2008. Communitary assay for assessment of effectiveness of strategies for prevention and control of human visceral leishmaniasis in the municipality of Feira de Santana, State of Bahia, Brazil. Epidemiologia e Serviços de Saúde 17, 97-106.

Deane, L. M., Deane, M. P., 1962. Visceral leishmaniasis in Brazil: geographical distribution and transmission. Revista do Instituto de Medicina Tropical de São Paulo 4, 198-212.

den Boer, M. L., Alvar, J., Davidson, R. N., Ritmeijer, K., Balasegaram, M., Sep. 2009. Developments in the treatment of visceral leishmaniasis. Expert Opinion on Emerging Drugs 14 (3), 395-410.

Dereure, J., El-Safi, S. H., Bucheton, B., Boni, M., Kheir, M. M., Davoust, B., Pratlong, F., Feugier, E., Lambert, M., Dessein, A., Dedet, J.-P., Oct. 2003. Visceral leishmaniasis in eastern Sudan: parasite identification in humans and dogs; host-parasite relationships. Microbes and Infection 5 (12), $1103-1108$.

Diekmann, O., Heesterbeek, J. A. P., Metz, J. A. J., 1990. On the definition and the computation of the basic reproduction ratio $R_{0}$ in models for infectious diseases in heterogeneous populations. Journal of Mathematical Biology 28, 365-382.

Dinesh, D. S., Das, P., Picado, A., Davies, C., Speybroeck, N., Boelaert, M., Coosemans, M., 2008a. The efficacy of indoor CDC light traps for collecting the sandfly Phlebotomus argentipes, vector of Leishmania donovani. Medical and Veterinary Entomology 22 (2), 120-123.

Dinesh, D. S., Das, P., Picado, A., Davies, C. R., Speybroeck, N., Ostyn, B., Boelaert, M., Coosemans, M., May 2008b. Long-lasting insecticidal nets fail at household level to reduce abundance of sandfly vector Phlebotomus argentipes in treated houses in Bihar (India). Tropical Medicine and International Health 13 (7), 953-958. 
Dorlo, T. P. C., Rijal, S., Ostyn, B., de Vries, P. J., Singh, R., Bhattarai, N., Uranw, S., Dujardin, J.-C., Boelaert, M., Beijnen, J. H., Huitema, A. D. R., Jun. 2014. Failure of miltefosine in visceral leishmaniasis is associated with low drug exposure. International Journal of Infectious Diseases $210(1), 146-153$.

dos Santos Marques, L. H., Gomes, L. I., da Rocha, I. C. M., da Silva, T. A. M., Oliveira, E., Morais, M. H. F., Rabello, A., Carneiro, M., Dec. 2012. Low parasite load estimated by $\mathrm{qPCR}$ in a cohort of children living in urban area endemic for visceral leishmaniasis in Brazil. PLoS Neglected Tropical Diseases 6 (12), e1955.

Downing, T., Imamura, H., Decuypere, S., Clark, T. G., Coombs, G. H., Cotton, J. A., Hilley, J. D., De Doncker, S., Maes, I., Mottram, J. C., Quail, M. A., Rijal, S., Sanders, M., Schonian, G., Stark, O., Sundar, S., Vanaerschot, M., Hertz-Fowler, C., Dujardin, J.-C., Berriman, M., Dec. 2011. Whole genome sequencing of multiple Leishmania donovani clinical isolates provides insights into population structure and mechanisms of drug resistance. Genome Research 21 (12), 2143-2156.

Dye, C., 1986. Population dynamics of mosquito-borne disease: effects of flies which bite some people more frequently than others. Transactions of the Royal Society of Tropical Medicine and Hygiene 80, 69-77.

Dye, C., Feb. 1988. The epidemiology of canine visceral leishmaniasis in southern France: classical theory offers another explanation of the data. Parasitology 96 (1), 19-24.

Dye, C., Aug. 1996. The logic of visceral leishmaniasis control. American Journal of Tropical Medicine and Hygiene 55 (2), 125-130.

Dye, C., Davies, C. R., Lainson, R., 1991. Communication among phlebotomine sandflies: a field study of domesticated Lutzomyia longipalpis populations in Amazonian Brazil. Animal Behaviour 42, 183-192.

Dye, C., Guy, M. W., Elkins, D. B., Wilkes, T. J., Killick-Kendrick, R., 1987. The life expectancy of phlebotomine sandflies: first field estimates from southern France. Medical and Veterinary Entomology 1, 417-425. 
Dye, C., Vidor, E., Dereure, J., 1993. Serological diagnosis of leishmaniasis: on detecting infection as well as disease. Epidemiology and Infection 103, 647-656.

Dye, C., Williams, B. G., 1993. Malnutrition, age and the risk of parasitic disease: visceral leishmaniasis revisited. Proceedings of the Royal Society B: Biological Sciences 254, 33-39.

Dye, C., Wolpert, D. M., 1988. Earthquakes, influenza and cycles of Indian kala-azar. Transactions of the Royal Society of Tropical Medicine and Hygiene $82,843-850$.

Edmunds, W. J., Medley, G. F., Nokes, D. J., 1999. Evaluating the costeffectiveness of vaccination programmes: A dynamic perspective. Statistic in Medicine 18, 3263-3282.

ELmojtaba, I. M., Mugisha, J. Y. T., Hashim, M. H. A., 2010a. Mathematical analysis of the dynamics of visceral leishmaniasis in the Sudan. Applied Mathematics and Computation 217, 2567-2578.

ELmojtaba, I. M., Mugisha, J. Y. T., Hashim, M. H. A., Jun. 2010b. Modelling the role of cross-immunity between two different strains of leishmania. Nonlinear Analysis: Real World Applications 11 (3), 2175-2189.

ELmojtaba, I. M., Mugisha, J. Y. T., Hashim, M. H. A., 2012. Vaccination model for visceral leishmaniasis with infective immigrants. Mathematical Methods in the Applied Sciences 36, 216-226.

Elnaiem, D. A., 1996. Use of pyrethroid impregnated bednets for the control of visceral leishmaniasis in eastern Sudan. Tech. Rep. WHO ID L3/1881/47, WHO.

Evans, T. G., Teixeira, M. J., McAuliffe, I. T., de Alencar Barros Vasconcelos, I., Vasconcelos, A. W., de Queiroz Sousa, A., de Oliveira Lima, J. W., Pearson, R. D., 1992. Epidemiology of visceral leishmaniasis in northeast Brazil. Journal of Infectious Diseases 166, 1124-1132.

Falcao, A. L., Falcao, A. R., Pinto, C. T., Gontijo, C. M. F., Falqueto, A., 1991. Effect of deltamethrin spraying on the sandfly populations in a focus of American cutaneous leishmaniasis. Memórias do Instituto Oswaldo Cruz 86, 399-404. 
Feliciangeli, M. D., Mazzarri, M. B., Blas, S. S., Zerpa, O., 2003a. Control trial of Lutzomyia longipalpis s.l. in the Island of Margarita, Venezuela. Tropical Medicine and International Health 8, 1131-1136.

Feliciangeli, M. D., Mazzarri, M. B., Campbell-Lendrum, D. H., Maroli, M., Maingon, R., 2003b. Cutaneous leishmaniasis vector control perspectives using lambdacyhalothrin residual house spraying in El Ingenio, Miranda State, Venezuela. Transactions of the Royal Society of Tropical Medicine and Hygiene 97, 641-646.

Felix de Lima, V. M., Ikeda, F. A., Rossi, C. u. N., Feitosa, M. M., de Oliveira Vasconcelos, R., Nunes, C. M., Goto, H., Jun. 2010. Diminished $\mathrm{CD} 4+/ \mathrm{CD} 25+\mathrm{T}$ cell and increased IFN- $\gamma$ levels occur in dogs vaccined with Leishmune in an endemic area for visceral leishmaniasis. Veterinary Immunology and Immunopathology 135 (3-4), 296-302.

Ferro, C., Morrison, A. C., Torres, M., Pardo, R., Wilson, M. L., Tesh, R. B., Sep. 1995. Age structure, blood-feeding behavior, and Leishmania chagasi infection in Lutzomyia longipalpis (Diptera: Psychodidae) at an endemic focus of visceral leishmaniasis in Colombia. Journal of Medical Entomology 32 (5), 618-629.

Foglia Manzillo, V., Di Muccio, T., Cappiello, S., Scalone, A., Paparcone, R., Fiorentino, E., Gizzarelli, M., Gramiccia, M., Gradoni, L., Oliva, G., May 2013. Prospective study on the incidence and progression of clinical signs in naïve dogs naturally infected by Leishmania infantum. PLoS Neglected Tropical Diseases 7 (5), e2225.

Franco, A. O., Davies, C. R., Mylne, A., Dedet, J.-P., Gallego, M., Ballart, C., Gramiccia, M., Gradoni, L., Molina, R., Galvez, R., Morillas-Marquez, F., Baron-Lopez, S., Pires, C. A., Afonso, M. O., Ready, P. D., Cox, J., Sep. 2011. Predicting the distribution of canine leishmaniasis in western Europe based on environmental variables. Parasitology 138 (14), 18781891.

Garlapati, R. B., Abbasi, I., Warburg, A., Poché, D., Poché, R., May 2012. Identification of bloodmeals in wild caught blood fed Phlebotomus argentipes (Diptera: Psychodidae) using cytochrome $b$ PCR and reverse line blotting in Bihar, India. Journal of Medical Entomology 49 (3), 515-521. 
Gavgani, A. S., Hodjati, M. H., Mohite, H., Davies, C. R., 2002. Effect of insecticide-impregnated dog collars on incidence of zoonotic visceral leishmaniasis in Iranian children: a matched-cluster randomised trial. The Lancet 360, 374-379.

Gebresilassie, A., Yared, S., Aklilu, E., Kirstein, O. D., Moncaz, A., Tekie, H., Balkew, M., Warburg, A., Hailu, A., Gebre-Michael, T., 2015. Host choice of Phlebotomus orientalis (Diptera: Psychodidae) in animal baited experiments: a field study in Tahtay Adiyabo district, northern Ethiopia. Parasites \& Vectors 8 (1), 190.

Ghosh, K., Mukhopadhyay, J., Desai, M. M., Senroy, S., Bhattacharya, A., Aug. 1999. Population ecology of Phlebotomus argentipes (Diptera: Psychodidae) in West Bengal, India. Journal of Medical Entomology 36 (5), 588-594.

Gidwani, K., Picado, A., Ostyn, B., Singh, S. P., Kumar, R., Khanal, B., Lejon, V., Chappuis, F., Boelaert, M., Sundar, S., Jan. 2011. Persistence of Leishmania donovani antibodies in past visceral leishmaniasis cases in India. Clinical and Vaccine Immunology 18 (2), 346-348.

Gradoni, L., Feb. 2015. Canine Leishmania vaccines: Still a long way to go. Veterinary Parasitology 208 (1-2), 94-100.

Guy, M. W., Killick-Kendrick, R., Gill, G. S., Rioux, J.-A., Bray, R. S., 1984. Ecology of leishmaniasis in the south of France. 19. Determination of the hosts of Phlebotomus ariasi Tonnoir, 1921 in the Cevennes by bloodmeal analyses. Annales de Parasitologie Humaine et Comparee (Paris) 59 (5), 449-458.

Hailu, A., Gramiccia, M., Kager, P. A., Dec. 2009. Visceral leishmaniasis in Aba-Roba, south-western Ethiopia: prevalence and incidence of active and subclinical infections. Annals of Tropical Medicine and Parasitology $103(8), 659-670$.

Hailu, A., Musa, A., Wasunna, M., Balasegaram, M., Yifru, S., Mengistu, G., Hurissa, Z., Hailu, W., Weldegebreal, T., Tesfaye, S., Makonnen, E., Khalil, E., Ahmed, O., Fadlalla, A., El-Hassan, A., Raheem, M., Mueller, M., Koummuki, Y., Rashid, J., Mbui, J., Mucee, G., Njoroge, S., Manduku, V., Musibi, A., Mutuma, G., Kirui, F., Lodenyo, H., Mutea, D., Kirigi, G., Edwards, T., Smith, P., Muthami, L., Royce, C., Ellis, S., Alobo, 
M., Omollo, R., Kesusu, J., Owiti, R., Kinuthia, J., for the Leishmaniasis East Africa Platform (LEAP) group, Oct. 2010. Geographical Variation in the Response of Visceral Leishmaniasis to Paromomycin in East Africa: A Multicentre, Open-Label, Randomized Trial. PLoS Neglected Tropical Diseases 4 (10), e709.

Hamad, S. H., Khalil, E. A. G., Musa, A. M., Ibrahim, M. E., Younis, B. M., Elfaki, M. E. E., El-Hassan, A. M., The Leishmaniasis Research Group, Aug. 2010. Leishmania donovani: Genetic diversity of isolates from Sudan characterized by PCR-based RAPD. Experimental parasitology 125 (4), 389-393.

Hartemink, N., Vanwambeke, S. O., Heesterbeek, H., Rogers, D., Morley, D., Pesson, B., Davies, C., Mahamdallie, S., Ready, P., Aug. 2011. Integrated mapping of establishment risk for emerging vector-borne infections: a case study of canine leishmaniasis in southwest France. PLoS One 6 (8), e20817.

Hasibeder, G., Dye, C., Carpenter, J., 1992. Mathematical modelling and theory for estimating the basic reproduction number of canine leishmaniasis. Parasitology 105 (1), 43-53.

Hasker, E., Kansal, S., Malaviya, P., Gidwani, K., Picado, A., Singh, R. P., Chourasia, A., Singh, A. K., Shankar, R., Menten, J., Wilson, M. E., Boelaert, M., Sundar, S., Feb. 2013. Latent Infection with Leishmania donovani in Highly Endemic Villages in Bihar, India. PLoS Neglected Tropical Diseases 7 (2), e2053.

Hasker, E., Malaviya, P., Gidwani, K., Picado, A., Ostyn, B., Kansal, S., Singh, R. P., Singh, O. P., Chourasia, A., Singh, A. K., Shankar, R., Wilson, M. E., Khanal, B., Rijal, S., Boelaert, M., Sundar, S., Jan. 2014. Strong association between serological status and probability of progression to clinical visceral leishmaniasis in prospective cohort studies in India and Nepal. PLoS Neglected Tropical Diseases 8 (1), e2657.

Hasker, E., Singh, S. P., Malaviya, P., Picado, A., Gidwani, K., Singh, R. P., Menten, J., Boelaert, M., Sundar, S., 2012. Visceral Leishmaniasis, Rural Bihar, India. Emerging Infectious Diseases 18 (10), 1662-1664.

Hotez, P. J., Alvarado, M., Basáñez, M.-G., Bolliger, I., Bourne, R., Boussinesq, M., Brooker, S. J., Brown, A. S., Buckle, G., Budke, C. M., Carabin, 
H., Coffeng, L. E., Fèvre, E. M., Fürst, T., Halasa, Y. A., Jasrasaria, R., Johns, N. E., Keiser, J., King, C. H., Lozano, R., Murdoch, M. E., O'Hanlon, S., Pion, S. D. S., Pullan, R. L., Ramaiah, K. D., Roberts, T., Shepard, D. S., Smith, J. L., Stolk, W. A., Undurraga, E. A., Utzinger, J., Wang, M., Murray, C. J. L., Naghavi, M., Jul. 2014. The global burden of disease study 2010: Interpretation and implications for the neglected tropical diseases. PLoS Neglected Tropical Diseases 8 (7), e2865.

Islam, S., Kenah, E., Bhuiyan, M. A. A., Rahman, K. M., Goodhew, B., Ghalib, C. M., Zahid, M. M., Ozaki, M., Rahman, M. W., Haque, R., Luby, S. P., Maguire, J. H., Martin, D., Bern, C., Aug. 2013. Clinical and immunological aspects of post-kala-azar dermal leishmaniasis in Bangladesh. American Journal of Tropical Medicine and Hygiene 89 (2), 345-353.

Jalouk, L., Al Ahmed, M., Gradoni, L., Maroli, M., 2007. Insecticide-treated bednets to prevent anthroponotic cutaneous leishmaniasis in Aleppo Governorate, Syria: results from two trials. Transactions of the Royal Society of Tropical Medicine and Hygiene 101, 360-367.

Joshi, A. B., Bhatt, L. R., Regmi, S., Ashford, R. W., 2003. An assessment of the effectiveness of insecticide spray in the control of visceral leishmaniasis in Nepal. Journal of the Nepal Health Research Council 1, 1-6.

Joshi, A. B., Das, M. L., Akhter, S., Chowdhury, R., Mondal, D., Kumar, V., Das, P., Kroeger, A., Boelaert, M., Petzold, M., Oct. 2009. Chemical and environmental vector control as a contribution to the elimination of visceral leishmaniasis on the Indian subcontinent: cluster randomized controlled trials in Bangladesh, India and Nepal. BMC Medicine 7 (1), 54.

Joshi, D. D., Sharma, M. C., Bhandari, S., 2006. Visceral leishmaniasis in Nepal during 1980-2006. Journal of Communicable Diseases 38, 139-148.

Joshi, R. D., Rai, R. N., 1994. Impact of DDT spraying on populations of $P$. argentipes and $P$. papatasi in Varanasi district, Uttar Pradesh. Journal of Communicable Diseases 26, 56-58.

Kassahun, A., Sadlova, J., Dvorak, V., Kostalova, T., Rohousova, I., Frynta, D., Aghova, T., Yasur-Landau, D., Lemma, W., Hailu, A., Baneth, G., Warburg, A., Volf, P., Votypka, J., 2015. Detection of Leishmania donovani and L. tropica in Ethiopian wild rodents. Acta Tropica 145, 39-44. 
Kaul, S. M., Sharma, R. S., Dey, K. P., Rai, R. N., Verghese, T., 1994. Impact of DDT indoor residual spraying on Phlebotomus argentipes in a kala-azar endemic village in eastern Uttar Pradesh. Bulletin of the World Health Organization 72 (1), 79-81.

Kedzierski, L., Evans, K. J., Jul. 2014. Immune responses during cutaneous and visceral leishmaniasis. Parasitology 141 (12), 1544-1562.

Keeling, M. J., Rohani, P., 2008. Modeling Infectious Diseases in Humans and Animals. Princeton University Press.

Kelly, D. W., Mustafa, Z., Dye, C., 1997. Differential application of lambdacyhalothrin to control the sandfly Lutzomyia longipalpis. Medical and Veterinary Entomology 11 (1), 13-24.

Kenubih, A., Dagnachew, S., Almaw, G., Abebe, T., Takele, Y., Hailu, A., Lemma, W., 2014. A preliminary survey of domestic animal visceral leishmaniasis and risk factors in North West Ethiopia. African Journal of Parasitological Research 1, 1-5.

Khalil, E. A. G., Weldegebreal, T., Younis, B. M., Omollo, R., Musa, A. M., Hailu, W., Abuzaid, A. A., Dorlo, T. P. C., Hurissa, Z., Yifru, S., Haleke, W., Smith, P. G., Ellis, S., Balasegaram, M., El-Hassan, A. M., Schoone, G. J., Wasunna, M., Kimutai, R., Edwards, T., Hailu, A., Jan. 2014. Safety and efficacy of single dose versus multiple doses of AmBisome for treatment of visceral leishmaniasis in eastern Africa: A randomised trial. PLoS Neglected Tropical Diseases 8 (1), e2613.

Killick-Kendrick, R., 1999. The biology and control of Phlebotomine sand flies. Clinics in Dermatology 17, 279-289.

Killick-Kendrick, R., Rioux, J.-A., 2002. Mark-release-recapture of sand flies fed on leishmanial dogs: The natural life-cycle of Leishmania infantum in Phlebotomus ariasi. Parassitologia (Rome) 44 (1-2), 67-71.

Kip, A. E., Balasegaram, M., Beijnen, J. H., Schellens, J. H. M., de Vries, P. J., Dorlo, T. P. C., Dec. 2014. Systematic review of biomarkers to monitor therapeutic response in leishmaniasis. Antimicrobial Agents and Chemotherapy 59 (1), 1-14. 
Kroeger, A., Avila, E. V., Morison, L., 2002. Insecticide impregnated curtains to control domestic transmission of cutaneous leishmaniasis in Venezuela: cluster randomised trial. British Medical Journal 325, 810-813.

Kumar, V., Kesari, S., Dinesh, D. S., Tiwari, A. K., Kumar, A. J., Singh, V. P., Das, P., 2009. A report on the indoor residual spraying (IRS) in the control of Phlebotomus argentipes, the vector of visceral leishmaniasis in Bihar (India): an initiative towards total elimination tareting 2015 (Series1). Journal of Vector-Borne Diseases 46, 225-229.

Lachaud, L., Bourgeois, N., Plourde, M., Leprohon, P., Bastien, P., Ouellette, M., Jan. 2009. Parasite Susceptibility to Amphotericin B in Failures of Treatment for Visceral Leishmaniasis in Patients Coinfected with HIV Type 1 and Leishmania infantum. Clinical Infectious Diseases 48 (2), e16$\mathrm{e} 22$.

Lachaud, L., Chabbert, E., Dubessay, P., Dereure, J., Lamothe, J., Dedet, J.-P., Bastien, P., 2002. Value of two PCR methods for the diagnosis of canine visceral leishmaniasis and the detection of asymptomatic carriers. Parasitology 125, 197-207.

Lainson, R., Rangel, E. F., 2005. Lutzomyia longipalpis and the ecoepidemiology of American visceral leishmaniasis, with particular reference to Brazil - A review. Memórias do Instituto Oswaldo Cruz 100, 811-827.

Lainson, R., Ward, R. D., Shaw, J. J., Apr. 1977. Experimental transmission of Leishmania chagasi, causative agent of neotropical visceral leishmaniasis, by the sandfly Lutzomyia longipalpis. Nature 266 (5603), 628-630.

Lane, R. P., Pile, M. M., Amerasinghe, F. P., 1990. Anthropophagy and aggregation behaviour of the sandfly Phlebotomus argentipes in Sri Lanka. Medical and Veterinary Entomology 4, 79-88.

Lee, B. Y., Bacon, K. M., Shah, M., Kitchen, S. B., Connor, D. L., Slayton, R. B., Mar. 2012. The economic value of a visceral leishmaniasis vaccine in Bihar state, India. American Journal of Tropical Medicine and Hygiene $86(3), 417-425$.

Lengeler, C., 2004. Insecticide-treated nets for malaria control: real gains. Bulletin of the World Health Organization 82, 84. 
Lira, R., Sundar, S., Makharia, A., Kenney, R. T., Gam, A., Saraiva, E. M., 1999. Evidence that the high incidence of treatment failures in Indian kalaazar is due to the emergence of antimony-resistant strains of Leishmania donovani. Journal of Infectious Diseases 180, 564-567.

Lukes, J., Mauricio, I. L., Schonian, G., Dujardin, J.-C., Soteriadou, K., Dedet, J.-P., Kuhls, K., Tintaya, K. W. Q., Jirku, M., Chocholova, E., Haralambous, C., Pratlong, F., Obornik, M., Horak, A., Ayala, F. J., Miles, M. A., 2007. Evolutionary and geographical history of the Leishmania donovani complex with a revisionof current taxonomy. PNAS 104, 9375-9380.

Macdonald, G., 1957. The Epidemiology and Control of Malaria. Oxford.

MacDonald, N., 1978. Time lags in biological models. Vol. 27. Springer Verlag.

Maia-Elkhoury, A. N. S., Alves, W. A., de Sousa-Gomes, M. L., de Sena, J. M., Luna, E. A., 2008. Visceral leishmaniasis in Brazil: trends and challenges. Cadernos de Saúde Pública 24, 2941-2947.

Mary, C., Faraut, F., Drougoul, M.-P., Xeridat, B., Schleinitz, N., Cuisenier, B., Dumon, H., Nov. 2006. Reference Values for Leishmania infantum parasitemia in different clinical presentations: Quantitative polymerase chain reaction for therapeutic monitoring and patient follow-up. American Journal of Tropical Medicine and Hygiene 75, 858-563.

Mary, C., Faraut, F., Lascombe, L., Dumon, H., 2004. Quantification of Leishmania infantum DNA by a real-time PCR assay with high sensitivity. Journal of Clinical Microbiology 42 (11), 5249-5255.

Mathers, C. D., Ezzati, M., Lopez, A. D., Nov. 2007. Measuring the burden of neglected tropical diseases: The global burden of disease framework. PLoS Neglected Tropical Diseases 1 (2), e114.

Matlashewski, G., Arana, B., Kroeger, A., Battacharya, S., Sundar, S., Das, P., Sinha, P. K., Rijal, S., Mondal, D., Zilberstein, D., Alvar, J., Mar. 2011. Visceral leishmaniasis: elimination with existing interventions. The Lancet Infectious Diseases 11 (4), 322-325. 
Mayrink, W., Magalhães, P. A., Batista, S. M., da Costa, C. A., 1971. Diagnosis of kala-azar. II. Study of Montenegro's test and detection of Leishmania in skin material from patients with kala-azar, before and after antimony treatment. Revista do Instituto de Medicina Tropical de São Paulo 13, 268-271.

Medley, G. F., Hollingsworth, T. D., Olliaro, P., Adams, E. R., In Press. Visceral leishmaniasis control: health-seeking, diagnostics and transmission. Nature (Suppl.).

Meheus, F., Balasegaram, M., Olliaro, P., Sundar, S., Rijal, S., Faiz, M. A., Boelaert, M., Sep. 2010. Cost-effectiveness analysis of combination therapies for visceral leishmaniasis in the Indian Subcontinent. PLoS Neglected Tropical Diseases 4 (9), e818.

Mejhed, H., Boussa, S., El Houda Mejhed, N., 2009. Development of mathematical models predicting the density of vectors: case of sandflies vectors of leishmaniasis. In: Proceedings of the 10th WSEAS International Conference on Mathematics and Computers in Biology and Chemistry. pp. $62-67$.

Melaku, Y., Collin, S. M., Keus, K., Gatluak, F., Ritmeijer, K., Davidson, R. N., 2007. Treatment of kala-azar in southern Sudan using a 17-day regimen of sodium stibogluconate combined with paramomycin: A retrospective comparison with 30-day sodium stibogluconate monotherapy. American Journal of Tropical Medicine and Hygiene 77, 89-94.

Miller, E., Warburg, A., Novikov, I., Hailu, A., Volf, P., Seblova, V., Huppert, A., Oct. 2014. Quantifying the contribution of hosts with different parasite concentrations to the transmission of visceral leishmaniasis in Ethiopia. PLoS Neglected Tropical Diseases 8 (10), e3288.

Molina, R., Amela, C., Nieto, J., San-Andres, M., Gonzales, F., Castillo, J. A., Lucientes, J., Alvar, J., 1994. Infectivity of dogs naturally infected with Leishmania infantum to colonized Phlebotomus perniciosus. Transactions of the Royal Society of Tropical Medicine and Hygiene 88 (4), 491-493.

Molina, R., Gradoni, L., Alvar, J., Oct. 2003. HIV and the transmission of Leishmania. Annals of Tropical Medicine and Parasitology 97 (Supplement-1), 29-45. 
Molina, R., Jiménez, M. I., Cruz, I., Iriso, A., Martín-Martín, I., Sevillano, O., Melero, S., Bernal, J., Nov. 2012. The hare (Lepus granatensis) as potential sylvatic reservoir of Leishmania infantum in Spain. Veterinary Parasitology 190 (1-2), 268-271.

Mondal, D., Alam, M. S., Karim, Z., Haque, R., Boelaert, M., Kroeger, A., 2008. Present situation of vector-control management in Bangladesh: a wake up call. Health Policy 87, 369-376.

Mondal, D., Huda, M. M., Karmoker, M. K., Ghosh, D., Matlashewski, G., Nabi, S. G., Kroeger, A., Jul. 2013. Reducing visceral leishmaniasis by insecticide impregnation of bed-nets, Bangladesh. Emerging Infectious Diseases 19 (7), 1131-1134.

Mondal, D., Nasrin, K. N., Huda, M. M., Kabir, M., Hossain, M. S., Kroeger, A., Thomas, T., Haque, R., Oct. 2010a. Enhanced case detection and improved diagnosis of PKDL in a kala-azar-endemic area of Bangladesh. PLoS Neglected Tropical Diseases 4 (10), e832.

Mondal, D., Singh, S. P., Kumar, N., Joshi, A., Sundar, S., Das, P., Siddhivinayak, H., Kroeger, A., Boelaert, M., Jan. 2009. Visceral leishmaniasis elimination programme in India, Bangladesh, and Nepal: Reshaping the case finding/case management strategy. PLoS Neglected Tropical Diseases 3 (1), e355.

Mondal, S., Bhattacharya, P., Ali, N., Aug. 2010b. Current diagnosis and treatment of visceral leishmaniasis. Expert Review of Anti-infective Therapy 8 (8), 919-944.

Moosa-Kazemi, S. H., Yaghoobi-Ershadir, M. R., Akhaven, A. A., Abdoli, H., Zahraei-Ramazani, A. R., Jafari, R., Houshmand, B., Nadim, A., Hosseini, M., 2007. Deltamethrin-impregnated bed nets and curtains in an anthroponotic cutaneous leishmaniasis control program in northeastern Iran. Annals of Saudi Medicine 27, 6-12.

Moral, L., Rubio, E. M., Moya, M., 2002. A leishmanin skin test survey in the human population of l'Alacantí region (Spain): implications for the epidemiology of Leishmania infantum infection in southern Europe. Transactions of the Royal Society of Tropical Medicine and Hygiene 96, 129-132. 
Morrison, A. C., Ferro, C., Morales, A., Tesh, R. B., Wilson, M. L., 1993 a. Dispersal of the sand fly Lutzomyia longipalpis (Diptera: Psychodidae) at an endemic focus of visceral leishmaniasis in Colombia. Journal of Medical Entomology 30 (2), 427-435.

Morrison, A. C., Ferro, C., Tesh, R. B., 1993b. Host preferences of the sand fly Lutzomyia longipalpis at an endemic focus of American visceral leishmaniasis in Colombia. American Journal of Tropical Medicine and Hygiene 49 (1), 68-75.

Mourão, M. V. A., Toledo, Jr, A., Gomes, L. I., Freire, V. V., Rabello, A., 2014. Parasite load and risk factors for poor outcome among children with visceral leishmaniasis. A cohort study in Belo Horizonte, Brazil, 2010-2011. Memórias do Instituto Oswaldo Cruz 109 (2), 147-153.

Mubayi, A., Castillo-Chavez, C., Chowell, G., Kribs-Zaleta, C., Ali Siddiqui, N., Kumar, N., Das, P., Jan. 2010. Transmission dynamics and underreporting of kala-azar in the Indian state of Bihar. Journal of Theoretical Biology 262 (1), 177-185.

Mueller, M., Ritmeijer, K., Balasegaram, M., Koummuki, Y., Santana, M. R., 2007. Unresponsiveness to AmBisome in some Sudanese patients with kalaazar. Transactions of the Royal Society of Tropical Medicine and Hygiene 101 (1), 19-24.

Mueller, Y. K., Nackers, F., Ahmed, K. A., Boelaert, M., Djoumessi, J.C., Eltigani, R., Gorashi, H. A., Hammam, O., Ritmeijer, K., Salih, N., Worku, D., Etard, J.-F., Chappuis, F., 2012. Burden of visceral leishmaniasis in villages of eastern Gedaref State, Sudan: An exhaustive crosssectional survey. PLoS Neglected Tropical Diseases 6 (11), e1872.

Mukhopadhyay, A. K., Hati, A. K., Chakraborty, S., Saxena, N. B., 1996. Effect of DDT on Phlebotomus sandflies in kala-azar endemic foci in West Bengal. The Journal of Communicable Diseases 28, 171-175.

Musa, A., Khalil, E., Hailu, A., Olobo, J., Balasegaram, M., Omollo, R., Edwards, T., Rashid, J., Mbui, J., Musa, B., Abuzaid, A. A., Ahmed, O., Fadlalla, A., El-Hassan, A., Mueller, M., Mucee, G., Njoroge, S., Manduku, V., Mutuma, G., Apadet, L., Lodenyo, H., Mutea, D., Kirigi, G., Yifru, S., Mengistu, G., Hurissa, Z., Hailu, W., Weldegebreal, T., Tafes, 
H., Mekonnen, Y., Makonnen, E., Ndegwa, S., Sagaki, P., Kimutai, R., Kesusu, J., Owiti, R., Ellis, S., Wasunna, M., Jun. 2012. Sodium Stibogluconate (SSG) \& Paromomycin Combination Compared to SSG for Visceral Leishmaniasis in East Africa: A Randomised Controlled Trial. PLoS Neglected Tropical Diseases 6 (6), e1674.

Musa, A. M., Khalil, E. A. G., Raheem, M. A., Zijlstra, E. E., Ibrahim, M. E., Elhassan, I. M., Mukhtar, M. M., El-Hassan, A. M., Dec. 2002. The natural history of Sudanese post-kala-azar dermal leishmaniasis: clinical, immunological and prognostic features. Annals of Tropical Medicine and Parasitology 96 (8), 765-772.

Musa, A. M., Khalil, E. A. G., Younis, B. M., Elfaki, M. E. E., Elamin, M. Y., Adam, A. O. A., Mohamed, H. A. A., Dafalla, M. M. M., Abuzaid, A. A., El-Hassan, A. M., 2013. Treatment-based strategy for the management of post-kala-azar dermal leishmaniasis patients in the Sudan. Journal of Tropical Medicine 2013 (1), Article ID 708391.

Nasreen, S. A., Hossain, M. A., Paul, S. K., Mahmud, M. C., Ahmed, S., Ghosh, S., Kobayashi, N., 2012. PCR-based detection of leishmania DNA in skin samples of post kala-azar dermal leishmaniasis patients from an endemic area of Bangladesh. Japan Journal of Infectious Diseases 65, 315317 .

Nogueira, F. S., Moreira, M. A. B., Borja-Cabrera, G. P., Santos, F. N., Menz, I., Parra, L. E., Xu, Z., Chu, H. J., Palatnik-de Sousa, C. B., Luvizotto, M. C. R., Sep. 2005. Leishmune $\AA$ vaccine blocks the transmission of canine visceral leishmaniasis. Vaccine 23 (40), 4805-4810.

Nunes, C. M., Lima, V. M. F. d., Paula, H. B. d., Perri, S. H. V., Andrade, A. M. d., Dias, F. E. F., Burattini, M. N., May 2008. Dog culling and replacement in an area endemic for visceral leishmaniasis in Brazil. Veterinary Parasitology 153 (1-2), 19-23.

Oliva, G., Nieto, J., Foglia Manzillo, V., Cappiello, S., Fiorentino, E., Di Muccio, T., Scalone, A., Moreno, J., Chicharro, C., Carrillo, E., Butaud, T., Guegand, L., Martin, V., Cuisinier, A.-M., McGahie, D., Gueguen, S., Canavate, C., Gradoni, L., Oct. 2014. A randomised, double-blind, controlled efficacy trial of the LiESP/QA-21 vaccine in naíve dogs exposed to 
two Leishmania infantum transmission seasons. PLoS Neglected Tropical Diseases 8 (10), e3213.

Oliva, G., Scalone, A., Foglia Manzillo, V., Gramiccia, M., Pagano, A., Di Muccio, T., Gradoni, L., Apr. 2006. Incidence and time course of Leishmania infantum infections examined by parasitological, serologic, and nested-PCR techniques in a cohort of naïve dogs exposed to three consecutive transmission seasons. Journal of Clinical Microbiology 44 (4), 13181322.

Oliveira da Silva, V., Borja-Cabrera, G. P., Correia Pontes, N. N., Paraguai de Souza, E., Luz, K. G., Palatnik, M., Palatnik-de Sousa, C. B., 2001. A phase III trial of efficacy of the FML-vaccine against canine kalaazar in an endemic area of Brazil (Sao Goncalo do Amaranto, RN). Vaccine 19, 1082-1092.

Olliaro, P., Guerin, P. M., Gerstl, S., Haaskjold, A. A., Rottingen, J.-A., Sundar, S., Nov. 2005. Treatment options for visceral leishmaniasis: a systematic review of clinical studies done in India, 1980-2004. The Lancet $5,763-774$.

Ostyn, B., Vanlerberghe, V., Picado, A., Dinesh, D. S., Sundar, S., Chappuis, F., Rijal, S., Dujardin, J.-C., Coosemans, M., Boelaert, M., Davies, C. R., Aug. 2008. Vector control by insecticide-treated nets in the fight against visceral leishmaniasis in the Indian subcontinent, what is the evidence? Tropical Medicine and International Health 13 (8), 1073-1085.

Otranto, D., Dantas-Torres, F., Jul. 2013. The prevention of canine leishmaniasis and its impact on public health. Trends in Parasitology 29 (7), 339-345.

Ozaki, M., Islam, S., Rahman, K. M., Rahman, A., Luby, S. P., Bern, C., Sep. 2011. Economic consequences of post-kala-azar dermal leishmaniasis in a rural Bangladeshi community. American Journal of Tropical Medicine and Hygiene 85 (3), 528-534.

Palatnik-de Sousa, C. B., Batista-de Melo, L. M., Borja-Cabrera, G. P., Palatnik, M., Lavor, C. C., Sep. 2004. Improving methods for epidemiological control of canine visceral leishmaniasis based on a mathematical 
model. Impact on the incidence of the canine and human disease. Anais da Academia Brasileira de Ciencias 76 (3), 583-593.

Palit, A., Bhattacharya, S. K., Kundu, S. N., 2006. Host preference of Phlebotomus argentipes and Phlebotomus papatasi in different biotopes of West Bengal, India. International Journal of Environmental Health Research 15 (6), 449-454.

Palit, A., Kishore, K., Sen, A. B., 1990. Gonotrophic cycles of Phlebotomus argentipes in nature in Bihar - preliminary experiences. Indian Journal of Parasitology 14 (2), 121-123.

Picado, A., Das, M. L., Kumar, V., Dinesh, D. S., Rijal, S., Singh, S. P., Das, P., Coosemans, M., Boelaert, M., Davies, C. R., Mar. 2010a. Phlebotomus argentipes seasonal patterns in India and Nepal. Journal of Medical Entomology 47 (2), 283-286.

Picado, A., Dash, A. P., Bhattacharya, S., Boelaert, M., Jul. 2012. Vector control interventions for visceral leishmaniasis elimination initiative in South Asia, 2005-2010. The Indian Journal of Medical Research 136 (1), $22-31$.

Picado, A., Ostyn, B., Rijal, S., Sundar, S., Singh, S. P., Chappuis, F., Das, M. L., Khanal, B., Gidwani, K., Hasker, E., Dujardin, J.-C., Vanlerberghe, V., Menten, J., Coosemans, M., Boelaert, M., Apr. 2015. Longlasting Insecticidal Nets to Prevent Visceral Leishmaniasis in the Indian Subcontinent; Methodological Lessons Learned from a Cluster Randomised Controlled Trial. PLoS Neglected Tropical Diseases 9 (4), e0003597.

Picado, A., Singh, S. P., Rijal, S., Sundar, S., Ostyn, B., Chappuis, F., Uranw, S., Gidwani, K., Khanal, B., Rai, M., Paudel, I. S., Das, M. L., Kumar, R., Srivastava, P., Dujardin, J.-C., Vanlerberghe, V., Andersen, E. W., Davies, C. R., Boelaert, M., 2010b. Longlasting insecticidal nets for prevention of Leishmania donovani infection in India and Nepal: paired cluster randomised trial. British Medical Journal 341, c6760.

Pourabbas, B., Ghadimi Moghadam, A., Pouladfar, G., Rezaee, Z., Alborzi, A., May 2013. Quantification of Leishmania infantum kinetoplast DNA for monitoring the response to meglumine antimoniate therapy in visceral 
leishmaniasis. American Journal of Tropical Medicine and Hygiene 88 (5), 868-871.

Quilez, J., Martínez, V., Woolliams, J. A., Sanchez, A., Pong-Wong, R., Kennedy, L. J., Quinnell, R. J., Ollier, W. E. R., Roura, X., Ferrer, L., Altet, L., Francino, O., Apr. 2012. Genetic control of canine leishmaniasis: Genome-wide association study and genomic selection analysis. PLoS One 7 (4), e35349.

Quinnell, R. J., Courtenay, O., 2009. Transmission, reservoir hosts and control of zoonotic visceral leishmaniasis. Parasitology 136, 1915-1934.

Quinnell, R. J., Courtenay, O., Davidson, S., Garcez, L., Lamothe, J., Ramos, P. K. S., Shaw, J. J., Shaw, M.-A., Dye, C., 2001. Detection of Leishmania infantum by PCR, serology and cellular immune response in a cohort study of Brazilian dogs. Parasitology 122, 253-261.

Quinnell, R. J., Courtenay, O., Garcez, L., Dye, C., Aug. 1997. The epidemiology of canine leishmaniasis: transmission rates estimated from a cohort study in Amazonian Brazil. Parasitology 115 (2), 143-156.

Quinnell, R. J., Dye, C., Shaw, J. J., 1992. Host preferences of the phlebotomine sandfly Lutzomyia longipalpis in Amazonian Brazil. Medical and Veterinary Entomology 6, 195-200.

Rahman, K. M., Islam, S., Rahman, M. W., Kenah, E., Galive, C. M., Zahid, M. M., Maguire, J., Rahman, M., Haque, R., Luby, S. P., Bern, C., Jan. 2010. Increasing incidence of post-kala-azar dermal leishmaniasis in a population-based study in Bangladesh. Clinical Infectious Diseases 50 (1), $73-76$.

Rebollar-Tellez, E. A., Hamilton, J. G. C., Ward, R. D., Mar. 2006. Genetic inherence of the response to human kairomones by two allopatric members of the Lutzomyia longipalpis complex. Physiological Entomology 31 (1), 94-97.

Reithinger, R., Brooker, S. J., Kolaczinski, J. H., 2007. Visceral leishmaniasis in eastern Africa - current status. Transactions of the Royal Society of Tropical Medicine and Hygiene 101, 1169-1170. 
Reithinger, R., Coleman, P. G., Alexander, B., Vieira, E. P., Assis, G., Davies, C. R., Jan. 2004. Are insecticide-impregnated dog collars a feasible alternative to dog culling as a strategy for controlling canine visceral leishmaniasis in Brazil? International Journal for Parasitology 34 (1), 5562.

Reyburn, H., Ashford, R., Mohsen, M., Hewitt, S., Rowland, M., Jul. 2000. A randomized controlled trial of insecticide-treated bednets and chaddars or top sheets, and residual spraying of interior rooms for the prevention of cutaneous leishmaniasis in Kabul, Afghanistan. Transactions of the Royal Society of Tropical Medicine and Hygiene 94 (4), 361-366.

Ribas, L. M., Zaher, V. L., Shimozako, H. J., Massad, E., 2013. Estimating the optimal control of zoonotic visceral leishmaniasis by the use of a mathematical model. TheScientificWorldJournal 2013 (3), 810380-6.

Rijal, S., Chappuis, F., Singh, R., Bovier, P. A., Acharya, P., Karki, B. M. S., Das, M. L., Desjeux, P., Loutan, L., Koirala, S., May 2003. Treatment of visceral leishmaniasis in south-eastern Nepal: decreasing efficacy of sodium stibogluconate and need for a policy to limit further decline. Transactions of the Royal Society of Tropical Medicine and Hygiene 97 (3), 350-354.

Rijal, S., Ostyn, B., Uranw, S., Rai, K., Bhattarai, N. R., Dorlo, T. P. C., Beijnen, J. H., Vanaerschot, M., Decuypere, S., Dhakal, S. S., Das, M. L., Karki, P., Singh, R., Boelaert, M., Dujardin, J.-C., May 2013. Increasing failure of miltefosine in the treatment of kala-azar in Nepal and the potential role of parasite drug resistance, reinfection, or noncompliance. Clinical Infectious Diseases 56 (11), 1530-1538.

Rijal, S., Yardley, V., Chappuis, F., Decuypere, S., Khanal, B., Singh, R., Boelaert, M., De Doncker, S., Croft, S. L., Dujardin, J.-C., Apr. 2007. Antimonial treatment of visceral leishmaniasis: are current in vitro susceptibility assays adequate for prognosis of in vivo therapy outcome? Microbes and Infection 9 (4), 529-535.

Ritmeijer, K., Davies, C. R., Van Zorge, R., Wang, S.-J., Schorscher, J., Dongu'du, S. I., Davidson, R. N., Feb. 2007. Evaluation of a mass distribution programme for fine-mesh impregnated bednets against visceral leishmaniasis in eastern Sudan. Tropical Medicine and International Health 12 (3), 404-414. 
Rock, K. S., Brand, S., Moir, J., Keeling, M. J., Jan. 2014. Dynamics of infectious diseases. Reports on Progress in Physics 77 (2), 026602.

Rock, K. S., le Rutte, E. A., de Vlas, S. J., Adams, E. R., Medley, G. F., Hollingsworth, T. D., Apr. 2015a. Uniting mathematics and biology for control of visceral leishmaniasis. Trends in Parasitology 31 (6), 1-9.

Rock, K. S., Stone, C. M., Hastings, I. M., Keeling, M. J., Torr, S. J., Chitnis, N., Mar. 2015b. Mathematical models of human African trypanosomiasis epidemiology. Advances in Parasitology 87 (3), 53-133.

Rodriguez-Barraquer, I., Buathong, R., Iamsirithaworn, S., Nisalak, A., Lessler, J., Jarman, R. G., Gibbons, R. V., Cummings, D. A. T., Jan. 2014. Revisiting Rayong: Shifting seroprofiles of dengue in Thailand and their implications for transmission and control. American Journal of Epidemiology 179 (3), 353-360.

Romero, G. A. S., Boelaert, M., Jan. 2010. Control of visceral leishmaniasis in Latin America - A systematic review. PLoS Neglected Tropical Diseases 4 (1), e584.

Ross, R., Feb. 1916. An application of the theory of probabilities to the study of a priori pathometry. Part I. Proceedings of the Royal Society A: Mathematical, Physical and Engineering Sciences 92 (638), 204-230.

Rossi, E., Bongiorno, G., Ciolli, E., Di Muccio, T., Scalone, A., Gramiccia, M., Gradoni, L., Maroli, M., Feb. 2008. Seasonal phenology, host-blood feeding preferences and natural Leishmania infection of Phlebotomus perniciosus (Diptera, Psychodidae) in a high-endemic focus of canine leishmaniasis in Rome province, Italy. Acta Tropica 105 (2), 158-165.

Sacks, D. L., Kenney, R. T., Kreutzer, R. D., Jaffe, C. L., Gupta, A. K., Sharma, M. C., Sinha, S. P., Neva, F. A., Saran, R., 1995. Indian kalaazar caused by Leishmania tropica. The Lancet 345, 959-961.

Salah, A. B., Smaoui, H., Mbarki, L., Anderson, R. M., Ismail, R. B., 1994. Development of a mathematical model on the dynamics of canine leishmaniasis transmission. Archives de l'Institut Pasteur de Tunis 71 (3-4), 431-438. 
Saraiva, E. M., Barbosa, A. d. F., Santos, F. N., Borja-Cabrera, G. P., Nico, D., Souza, L. O. P., Mendes-Aguiar, C. d. O., de Souza, E. P., Fampa, P., Parra, L. E., Menz, I., Dias, J. G., de Oliveira, S. M., Palatnik-de Sousa, C. B., Mar. 2006. The FML-vaccine (Leishmune®) against canine visceral leishmaniasis: A transmission blocking vaccine. Vaccine 24 (13), 2423-2431.

Schaefer, K. U., Kurtzhals, J. A., Gachihi, G. S., Muller, A. S., Kager, P. A., 1995. A prospective sero-epidemiological study of visceral leishmaniasis in Baringo District, Rift Valley Province, Kenya. Transactions of the Royal Society of Tropical Medicine and Hygiene 89, 471-475.

Seaman, J., Pryce, D., Sondorp, H. E., Moody, A., Bryceson, A. D., Davidson, R. N., 1993. Epidemic visceral leishmaniasis in Sudan: a randomized trial of aminosidine plus sodium stibogluconate versus sodium stibogluconate alone. Journal of Infectious Diseases 168, 715-720.

Seifert, K., Perez-Vitoria, F. J., Stettler, M., Sanchez-Canete, M. P., Castanys, S., Gamarro, F., Croft, S. L., 2007. Inactivation of the miltefosine transporter, LdMT, causes miltefosine resistance that is conferred to the amastigote stage of Leishmania donovani and persists in vivo. International Journal of Antimicrobial Agents 30, 229-235.

Sharma, N. L., Mahajan, V. K., Negi, A. K., Verma, G. K., 2009. The rK39 immunochromatic dipstick testing: a study for K39 seroprevalence in dogs and human leishmaniasis patients for possible animal reservoir of cutaneus and visceral leishmaniasis in endemic focus of Satluj river valley of Himachal Pradesh (India). Indian Journal of Dermatology, Venereology and Leprology 75, 52-55.

Sherlock, I. A., 1996. Ecological Interactions of Visceral Leishmaniasis in the State of Bahia, Brazil. Memórias do Instituto Oswaldo Cruz 91 (6), 671-683.

Singh, O. P., Hasker, E., Sacks, D., Boelaert, M., Sundar, S., Apr. 2014. Asymptomatic Leishmania infection: A new challenge for Leishmania control. Clinical Infectious Diseases 58 (10), 1424-1429.

Singh, R., Lal, S., Saxena, V. K., Aug. 2008. Breeding ecology of visceral leishmaniasis vector sandfly in Bihar state of India. Acta Tropica 107 (2), $117-120$. 
Singh, R. P., Picado, A., Alam, S., Hasker, E., Singh, S. P., Ostyn, B., Chappuis, F., Sundar, S., Boelaert, M., Aug. 2012. Post-kala-azar dermal leishmaniasis in visceral leishmaniasis-endemic communities in Bihar, India. Tropical Medicine and International Health 17 (11), 1345-1348.

Siriwardana, H. V. Y. D., Chandrawansa, P. H., Sirimanna, G., Karunaweera, N. D., Aug. 2012. Leishmaniasis in Sri Lanka: a decade old story. Sri Lankan Journal of Infectious Diseases 2 (2), 2-12.

Smith, D. L., Battle, K. E., Hay, S. I., Barker, C. M., Scott, T. W., McKenzie, F. E., Apr. 2012. Ross, Macdonald, and a theory for the dynamics and control of mosquito-transmitted pathogens. PLoS Pathogens 8 (4), e1002588.

Smith, D. L., McKenzie, F. E., Snow, R. W., Hay, S. I., 2007. Revisiting the basic reproductive number for malaria and its implications for malaria control. PLoS Biology 5 (3), e42.

Smith, D. L., Perkins, T. A., Reiner, R. C., Barker, C. M., Niu, T., Chaves, L. F., Ellis, A. M., George, D. B., Le Menach, A., Pulliam, J. R. C., Bisanzio, D., Buckee, C., Chiyaka, C., Cummings, D. A. T., Garcia, A. J., Gatton, M. L., Gething, P. W., Hartley, D. M., Johnston, G., Klein, E. Y., Michael, E., Lloyd, A. L., Pigott, D. M., Reisen, W. K., Ruktanonchai, N., Singh, B. K., Stoller, J., Tatem, A. J., Kitron, U., Godfray, H. C. J., Cohen, J. M., Hay, S. I., Scott, T. W., Mar. 2014. Recasting the theory of mosquito-borne pathogen transmission dynamics and control. Transactions of the Royal Society of Tropical Medicine and Hygiene 108 (4), 185-197.

Solano-Gallego, L., Koutinas, A., Miró, G., Cardoso, L., Pennisi, M. G., Ferrer, L., Bourdeau, P., Oliva, G., Baneth, G., Oct. 2009. Directions for the diagnosis, clinical staging, treatment and prevention of canine leishmaniosis. Veterinary Parasitology 165 (1-2), 1-18.

Solano-Gallego, L., Morell, P., Arboix, M., Alberola, J., Ferrer, L., Feb. 2001. Prevalence of Leishmania infantum infection in dogs living in an area of canine leishmaniasis endemicity using PCR on several tissues and serology. Journal of Clinical Microbiology 39 (2), 560-563.

Srinivasan, R., Panicker, K. N., 1992. Seasonal abundance, natural survival and resting behaviour of Phlebotomus papatasi (Diptera: Phlebotomidae) in Pondicherry. Indian Journal of Medical Research A 95, 207-211. 
Stauch, A., Duerr, H.-P., Dujardin, J.-C., Vanaerschot, M., Sundar, S., Eichner, M., Dec. 2012. Treatment of visceral leishmaniasis: Model-based analyses on the spread of antimony-resistant L. donovani in Bihar, India. PLoS Neglected Tropical Diseases 6 (12), e1973.

Stauch, A., Duerr, H.-P., Picado, A., Ostyn, B., Sundar, S., Rijal, S., Boelaert, M., Dujardin, J.-C., Eichner, M., Apr. 2014. Model-based investigations of different vector-related intervention strategies to eliminate visceral leishmaniasis on the Indian subcontinent. PLoS Neglected Tropical Diseases 8 (4), e2810.

Stauch, A., Sarkar, R. R., Picado, A., Ostyn, B., Sundar, S., Rijal, S., Boelaert, M., Dujardin, J.-C., Duerr, H.-P., Nov. 2011. Visceral leishmaniasis in the Indian subcontinent: modelling epidemiology and control. PLoS Neglected Tropical Diseases 5 (11), e1405.

Sudarshan, M., Singh, T., Singh, A. K., Chourasia, A., Singh, B., Wilson, M. E., Chakravarty, J., Sundar, S., Dec. 2014. Quantitative PCR in Epidemiology for Early Detection of Visceral Leishmaniasis Cases in India. PLoS Neglected Tropical Diseases 8 (12), e3366.

Sudarshan, M., Sundar, S., Sep. 2014. Parasite load estimation by qPCR differentiates between asymptomatic and symptomatic infection in Indian cisceral leishmaniasis. Diagnostic Microbiology and Infectious Disease 80 (1), 40-42.

Sudarshan, M., Weirather, J. L., Wilson, M. E., Sundar, S., Jul. 2011. Study of parasite kinetics with antileishmanial drugs using real-time quantitative PCR in Indian visceral leishmaniasis. Journal of Antimicrobial Chemotherapy 66 (8), 1751-1755.

Sultana, A., Zakaria, S. M., Bhuiyan, S. I., Habib, A., Dey, S. K., Rahman, M., Basher, A., Jul. 2012. Spectrum of skin lesions of post-kala-azar dermal leishmaniasis in kala-azar endemic areas of Bangladesh. Mymensingh Medical Journal 21 (3), 529-532.

Sundar, S., 2001. Drug resistance in Indian visceral leishmaniasis. Tropical Medicine and International Health 6, 849-854. 
Sundar, S., Jha, T. K., Mishra, M., Singh, V. P., Buffels, R., 2003. SingleDose Liposomal Amphotericin B in the Treatment of Visceral Leishmaniasis in India: A Multicenter Study. Clinical Infectious Diseases 37, 800-804.

Sundar, S., Mehta, H., Suresh, A. V., Singh, S. P., Rai, M., Murray, H. W., 2004. Amphotericin B Treatment for Indian Visceral Leishmaniasis: Conventional versus Lipid Formulations. Clinical Infectious Diseases 38, 377383.

Sundar, S., More, D. K., Singh, M. K., Singh, V. P., Sharma, S., Makharia, A., Kumar, P. C., Murray, H. W., 2000. Failure of pentavalent antimony in visceral leishmaniasis in India: report from the center of the Indian epidemic. Clinical Infectious Diseases 31, 1104-1107.

Sundar, S., Rai, M., Chakravarty, J., Agarwal, D., Agrawal, N., Vaillant, M., Olliaro, P., Murray, H. W., Oct. 2008. New Treatment Approach in Indian Visceral Leishmaniasis: Single-Dose Liposomal Amphotericin B Followed by Short-Course Oral Miltefosine. Clinical Infectious Diseases 47 (8), 10001006.

Sundar, S., Singh, A., Rai, M., Prajapati, V. K., Singh, A. K., Ostyn, B., Boelaert, M., Dujardin, J.-C., Chakravarty, J., Jul. 2012. Efficacy of miltefosine in the treatment of visceral leishmaniasis in India after a decade of use. Clinical Infectious Diseases 55 (4), 543-550.

Svobodova, M., Votypka, J., Nicolas, L., Volf, P., 2003. Leishmania trop$i c a$ in the black rat (Rattus rattus): persistence and transmission from asymptomatic host to sand fly vector Phlebotomus sergenti. Microbes and Infection 5, 361-364.

Tayeh, A., Jalouk, L., Al-Khiami, A. M., 1997. A cutaneous leishmaniasis control trial using pyrethroid-impregnated bednets in villages near Aleppo, Syria. Tech. Rep. WHO/LEISH/97.41, WHO.

Thakur, C. P., Kanyok, T. P., Pandey, A. K., Sinha, G. P., Zaniewski, A. E., Houlihan, H. H., Olliaro, P., Jul. 2000. A prospective randomized, comparative, open-label trial of the safety and efficacy of paromomycin (aminosidine) plus sodium stibogluconate versus sodium stibogluconate alone for the treatment of visceral leishmaniasis. Transactions of the Royal Society of Tropical Medicine and Hygiene 94 (4), 429-431. 
Thakur, C. P., Kumar, K., Aug. 1992. Post kala-azar dermal leishmaniasis: a neglected aspect of kala-azar control programmes. Annals of Tropical Medicine and Parasitology 86 (4), 355-359.

Tiwary, P., Kumar, D., Mishra, M., Singh, R. P., Rai, M., Sundar, S., Apr. 2013. Seasonal variation in the prevalence of sand flies infected with Leishmania donovani. PLoS One 8 (4), e61370.

Topno, R. K., Das, V. N. R., Ranjan, A., Pandey, K., Singh, D., Kumar, N., Siddiqui, N. A., Singh, V. P., Kesari, S., Bimal, S., Kumar, A. J., Meena, C., Kumar, R., Das, P., Sep. 2010. Asymptomatic infection with visceral leishmaniasis in a disease-endemic area in Bihar, India. American Journal of Tropical Medicine and Hygiene 83 (3), 502-506.

Travi, B. L., Montoya, J., Gallego, J., Jaramillo, C., Llano, R., Velez, I. D., 1996. Bionomics of Lutzomyia evansi (Diptera: Psychodidae) vector of visceral leishmaniasis in Northern Colombia. Journal of Medical Entomology $33,278-285$.

Travi, B. L., Tabares, C. J., Cadena, H., Ferro, C., Osorio, Y., 2001. Canine visceral leishmaniasis in Colombia: Relationship between clinical and parasitologic status and infectivity for sand flies. American Journal of Tropical Medicine and Hygiene 64, 119-124.

Uranw, S., Ostyn, B., Rijal, A., Devkota, S., Khanal, B., Menten, J., Boelaert, M., Rijal, S., Dec. 2011. Post-kala-azar dermal leishmaniasis in Nepal: A retrospective cohort study (2000-2010). PLoS Neglected Tropical Diseases 5 (12), e1433.

van Griensven, J., Balasegaram, M., Meheus, F., Alvar, J., Lynen, L., Boelaert, M., Mar. 2010. Combination therapy for visceral leishmaniasis. The Lancet Infectious Diseases 10 (3), 184-194.

Verma, N., Singh, D., Pandey, K., Das, V. N. R., Lal, C. S., Verma, R. B., Sinha, P. K., Das, P., Nov. 2013. Comparative evaluation of PCR and imprint smear microscopy analyses of skin biopsy specimens in diagnosis of macular, papular, and mixed papulo-nodular lesions of post-kala-azar dermal leishmaniasis. Journal of Clinical Microbiology 51 (12), 4217-4219.

Verma, S., Kumar, R., Katara, G. K., Singh, L. C., Negi, N. S., Ramesh, V., Salotra, P., Apr. 2010. Quantification of parasite load in clinical samples of 
leishmaniasis patients: IL-10 level correlates with parasite load in visceral leishmaniasis. PLoS One 5 (4), e10107.

Viana, G. M. C., Nascimento, M. D. S. B., Diniz Neto, J. A., Rabelo, E. M. F., Binda Júnior, J. R., Santos Júnior, O. M., Santos, A. C., Galvão, C. S., Guimarães, R. S., 2011. Anti-Leishmania titers and positive skin tests in patients cured of kala-azar. Brazilian Journal of Medical and Biological Research 44 (1), 62-65.

Weirather, J. L., Jeronimo, S. M. B., Gautam, S., Sundar, S., Kang, M., Kurtz, M. A., Haque, R., Schriefer, A., Talhari, S., Carvalho, E. M., Donelson, J. E., Wilson, M. E., Oct. 2011. Serial quantitative PCR assay for detection, species discrimination, and quantification of Leishmania spp. in human samples. Journal of Clinical Microbiology 49 (11), 3892-3904.

WHO, 1982. Manual on environmental management for mosquito control. Tech. Rep. 66.

WHO, 2002. Global Burden of Disease in 2002: data sources, methods and results. Tech. Rep. 54, WHO.

WHO, 2010a. Control of the leishmaniasis. Tech. Rep. 949, WHO.

WHO, 2010b. Monitoring and evaluation tool kit for indoor residual spraying. Tech. rep., WHO.

WHO, Feb. 2013. Sustaining the drive to overcome the global impact of neglected tropical diseases. Tech. Rep. WHO/HTM/NTD/2013.1, WHO.

Williams, B. G., Dye, C., 1997. Infectious disease persistence when transmission varies seasonally. Mathematical Biosciences 145, 7-88.

Woolhouse, M. E., Dye, C., Etard, J.-F., Smith, T., Charlwood, J. D., Garnett, G. P., Hagan, P., Hii, J. L., Ndhlovu, P. D., Quinnell, R. J., Watts, C. H., Chandiwana, S. K., Anderson, R. M., Jan. 1997. Heterogeneities in the transmission of infectious agents: implications for the design of control programs. PNAS 94 (1), 338-342.

Wylie, C. E., Carbonell-Antoñanzas, M., Aiassa, E., Dhollander, S., Zagmutt, F. J., Brodbelt, D. C., Solano-Gallego, L., Nov. 2014. A systematic review of the efficacy of prophylactic control measure for naturally- 
occurring canine leishmaniosis, part I: Vaccinations. Preventive Veterinary Medicine 117 (1), 7-18.

Yaghoobi-Ershadi, M. R., Moosa-Kazemi, S. H., Zahraei-Ramazani, A. R., Jalai-Zand, A. R., Akhaven, A. A., Arandian, M. H., Abdoli, H., Houshmand, B., Nadim, A., Hosseini, M., 2006. Evaluation of deltamethrinimpregnated bed nets and curtains for control of zoonotic cutaneous leishmaniasis in a hyperendemic area of Iran. Bulletin de la societe de pathologie exotique $99,43-48$.

Zhi-Biao, X., 1989. Present situation of visceral leishmaniasis in China. Parasitology Today 5, 224-228.

Zijlstra, E. E., El-Hassan, A. M., Ismael, A., Ghalib, H. W., Dec. 1994. Endemic kala-azar in Eastern Sudan: A longitudinal study on the incidence of clinical and subclinical infection and post-kala-azar demal leishmaniasis. American Journal of Tropical Medicine and Hygiene 51 (6), 826-836.

Zijlstra, E. E., Musa, A. M., Khalil, E. A. G., El-Hassan, I. M., El-Hassan, A. M., Feb. 2003. Post-kala-azar dermal leishmaniasis. The Lancet Infectious Diseases 3 (2), 87-98. 